\title{
Thermal Conductivity of Architected Cellular Metamaterials
}

\author{
Armin Mirabolghasemi \\ Department of Bioresource Engineering \\ McGill University, Montreal \\ Quebec, Canada \\ A thesis submitted to McGill University \\ in partial fulfillment of the requirements of the degree of \\ Master of Science
}

December 2018

(C) Armin Mirabolghasemi, 2018 
Dedicated to my family $\square$

Maman nafas, Maryam joon and dawsh Pejman 
"When modern man builds large load-bearing structures, he uses dense solids: steel, concrete, glass. When nature does the same, she generally uses cellular materials: wood, bone, coral. There must be good reasons for it."

M.F.Ashby 


\section{Table of Contents}

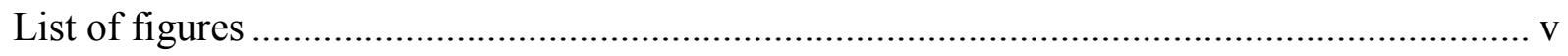

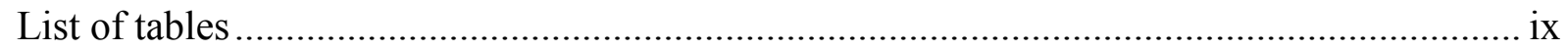

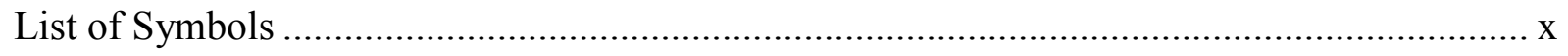

List of Abbreviations........................................................................................................

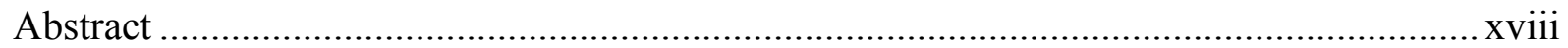

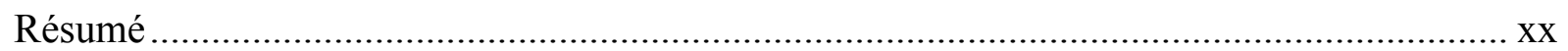

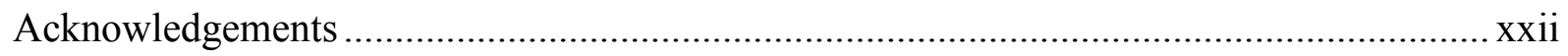

Preface and Contribution of Authors ...................................................................................

1 Literature Review and General Introduction........................................................... 1

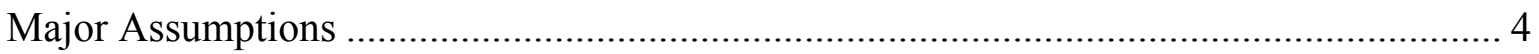

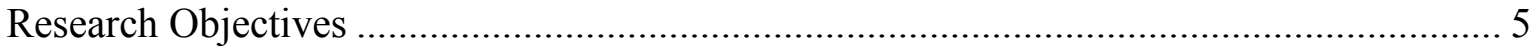

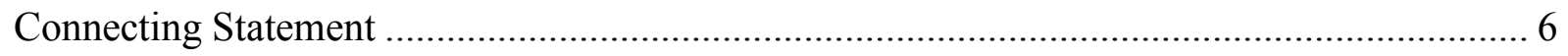

2 Thermal Conductivity of Architected Cellular Metamaterials ........................................ 7

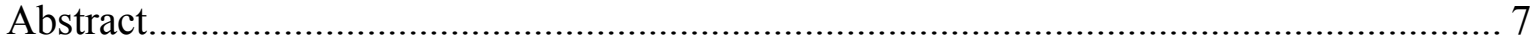

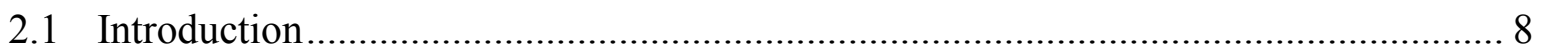

2.2 CAD Design of Architected Cellular Metamaterials ........................................... 10

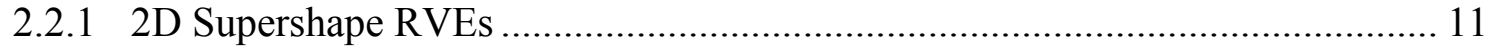

2.2.2 3D Thin-walled Open Lattices .............................................................. 13

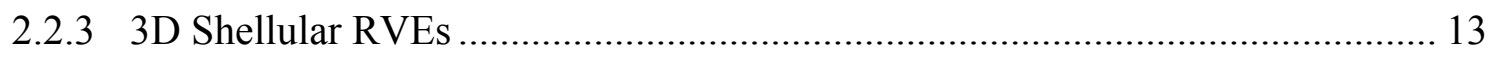

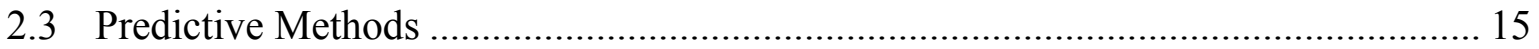

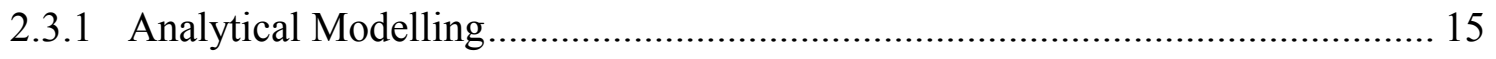

2.3.2 Computational Standard Mechanics Homogenization ...................................... 17

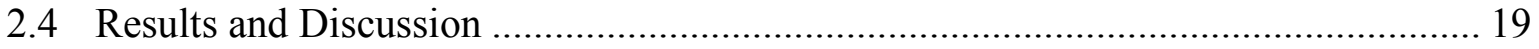

2.5 Functionally Graded Cellular Materials............................................................ 36

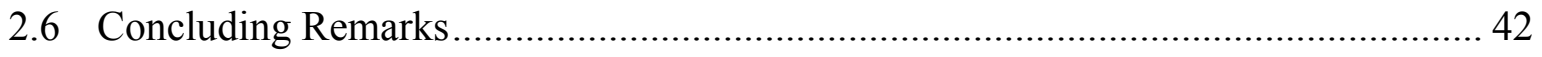

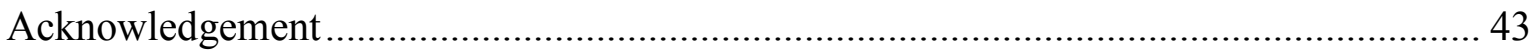

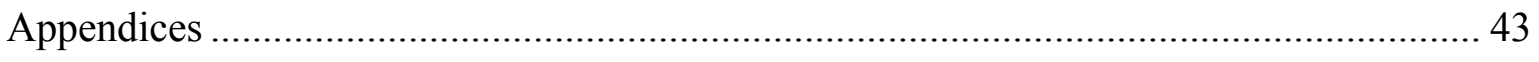

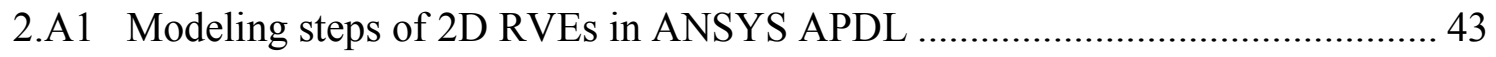

2.A2 Steps for 3D modeling of a 3D Shellular RVE........................................... 43 
2.A3 Verification of the numerical homogenization results.

2.A4 Effect of $m$ and $n$ on the relation between effective principal thermal conductivities of thin-walled open lattices.......................................................... 45

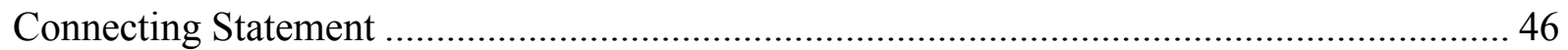

3 Thermal Conductivity of Conformally Perforated Shellular Metamaterials ....................... 47

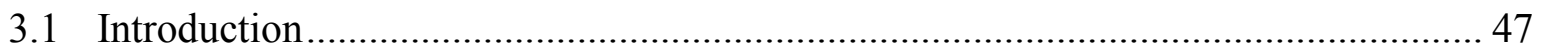

3.2 Conformal Mapping ............................................................................................ 48

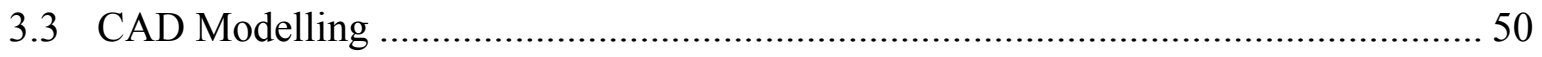

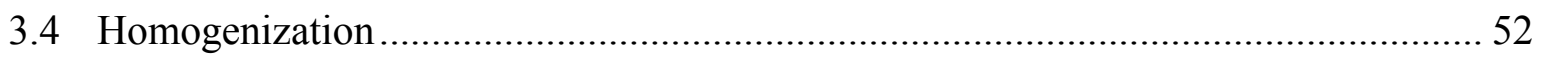

3.5 Results and Discussion ..................................................................................... 53

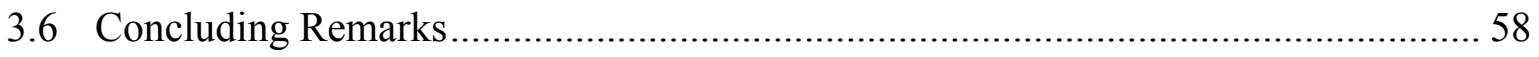

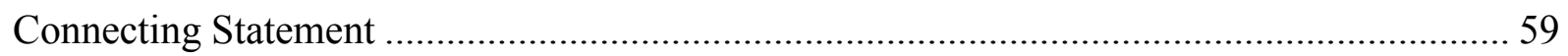

4 Thermal Conductivity of FCC and SC Periodic Truss Materials ...................................... 60

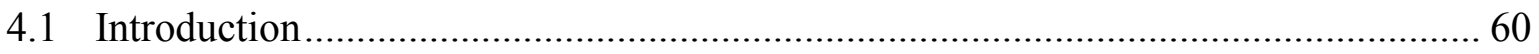

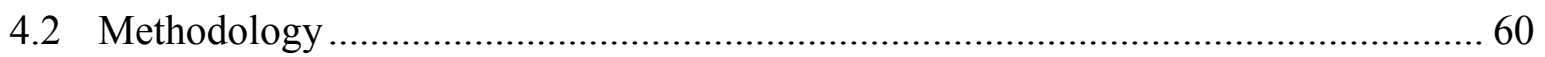

4.2.1 CAD Modeling of FCC and SC Periodic Truss Architectures............................. 61

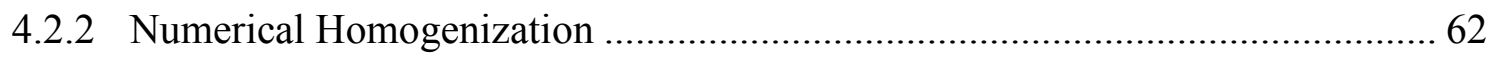

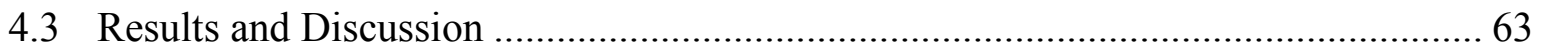

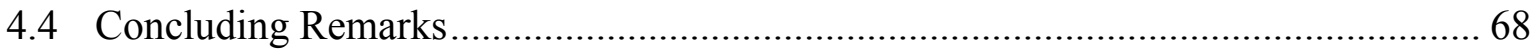

5 Summary, Conclusion and Suggested Future Works ………………….......................... 69

Suggested Future Works.......................................................................................... 70

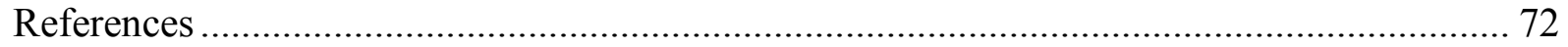




\section{LIST OF FIGURES}

Figure 2.1: Sample RVEs of 2D architected cellular metamaterials for different topological parameters: (a) $\rho_{r}=0.88, S=1$ and $\beta=0^{\circ}$ and (b) $\rho_{r}=0.62$, $S=1.5$ and $\beta=45^{\circ}$.

Figure 2.2: (a) Local coordinate system used for the creation of $2 \mathrm{D}$ supershape pores on each face of 3D RVE, (b) Possible thin-walled open lattice modeled in SolidWorks, and (c) Schematic view of a thin-walled open lattice considered in this study.

Figure 2.3: $3 \times 3 \times 3$ TPMS cells together with two Shellular RVEs at 0.02 and 0.10 relative thicknesses: (a) Schwarz D, (b) Gyroid, (c) Schwarz $\mathrm{P}$ with $f=0$, and (d) Schwarz $\mathrm{P}$ with $f=0.8$.

Figure 2.4: Schematic view of the thermal resistant elements for: (a) Horizontal isothermal lines where $\delta R^{y}=\left(\frac{1}{\delta R_{m}^{y}}+\frac{1}{\delta R_{i}^{y}}\right)^{-1}$ and,$R^{y}=\sum \delta R^{y}$ and (b)

Vertical adiabatic lines with $\delta R^{y}=\delta R_{m}^{y}+\delta R_{i}^{y}$ and $R^{y}=\left(\sum \frac{1}{\delta R^{y}}\right)^{-1} \ldots \ldots . . .16$

Figure 2.5: Effective thermal conductivity of air-filled $\left(\mathrm{k}_{\text {air }}=0.0263 \mathrm{~W} / \mathrm{mK}\right)$ and empty $\left(\mathrm{k}_{\mathrm{i}}=0\right)$ cellular materials for a square pore shape versus the thermal conductivity of the solid matrix (log-log scale).....

Figure 2.6: Theoretical upper and lower bounds along with computational homogenized values of the thermal conductivity along y direction, normalized by the thermal conductivity of the solid matrix: (a) rectangular, and (b) elliptic pores.

Figure 2.7: Normalized effective principal thermal conductivities predicted by standard mechanics homogenization for 2D supershape RVEs.

Figure 2.8: Two RVEs with relative densities below 0.2 .

Figure 2.9: An example of an RVE with $\rho_{r}=0.132$ and a highly anisotropic effective thermal conductivity $\left(K_{1}^{e f f} / K_{m}=0.122\right.$ and $\left.K_{2}^{e f f} / K_{m}=0.002\right)$. Rectangular pore's specifications are: 19.1 aspect ratio and $14^{\circ}$ rotation angle. 
Figure 2.10: Effective principal thermal conductivities of $2 \mathrm{D}$ supershape RVEs for different sets of topological parameters: a) $[n=3, m=6]$, b) $[n=3, m=3]$, c) $n=0$, d) $[\mathrm{n}=-3, \mathrm{~m}=6]$, and e) $[\mathrm{n}=-3, \mathrm{~m}=3]$ 25

Figure 2.11: Effective off-diagonal term in the thermal conductivity tensor for $2 \mathrm{D}$ supershape RVEs with respect to the relative density obtained by standard mechanics homogenization. 26

Figure 2.12: Using the Mohr's circle to calculate the thermal conductivity components for a rotated anisotropic material. 26

Figure 2.13: Assembling or disassembling a general thin-walled open lattice RVE 29

Figure 2.14: Effective $K_{x x}$ and $K_{x y}$ normalized by the base material's thermal conductivity times the relative wall thickness, for the thin-walled open lattices based on: (a) supershape parameter $\mathrm{m}=1$ and (b) supershape parameter $\mathrm{m}=2$. Only the face of the 3D RVE in the $\mathrm{x}-\mathrm{y}$ plane is shown.

Figure 2.15: Deviation of the results of Eq. (2.9) from 3D standard mechanics homogenization for thin-walled open lattice with supershape parameters set as $[n=-3, m=3, S=1, \beta=0]$.

Figure 2.16: Effective thermal conductivity of Shellular materials, normalized by thermal conductivity of the solid matrix, with respect to relative density.

Figure 2.17: Normalized effective thermal conductivity of Shellular materials with respect to relative thickness: (a) G, D and P Shellular materials with $f=0$, and (b) P Shellular materials with different $f$ values. 34

Figure 2.18: Maximum vs. minimum effective principal thermal conductivity of 2D supershape cellular, 3D thin-walled open lattices, and Shellular materials for different relative densities. 35

Figure 2.19: FDM 3D printed unit cells of some 2D and 3D RVEs presented in this paper: (a) 2D supershape RVEs, (b) Thin-walled open lattice, (c) Gyroid or G type Shellular $(f=0)$, (d) Diamond or D type Shellular $(f=0)$, (e) Primitive or $\mathrm{P}$ type Shellular with $f=0$, (f) $\mathrm{P}$ type Shellular with $f=0.4$ and (g) P type Shellular with $f=0.8$. All 3D RVEs are $4 \times 4 \times 4 \mathrm{~cm}^{3}$ 36

Figure 2.20: Schematic representation of an FGCM subjected to thermal loading. 37 
Figure 2.21: Numerical simulation results of the square FGCM introduced in Fig. 2.20 for different $\beta_{1}$ to $\beta_{4}$ values. (a) Heat flow rate through the right edge $\left(\mathrm{Q}_{\mathrm{r}}\right)$, (b) Heat flow rate through the top edge $\left(\mathrm{Q}_{\mathrm{t}}\right)$, and (c) Maximum temperature throughout the FGCM. Stars on the color bars indicates $\mathrm{Q}_{\mathrm{r}}, \mathrm{Q}_{\mathrm{t}}$ and $T_{\max }$ for the solid part under the same boundary conditions. For points $\mathrm{A} \sim \mathrm{E}$, please refer to Table 2.5 .

Figure 2.22: Heat flow rates through the top and right edges together with the maximum temperature as a function of pore's rotation angle, in the part made by uniform cellular materials $\left(\beta_{1}=\beta_{2}=\beta_{3}=\beta_{4}\right)$.

Figure 2.A.1: Modeling steps of 2D RVEs in ANSYS APDL: (a) keypoints, (b) spline segments passing through the keypoints, (c) pore area confined by the splines, without scaling and rotation, (d) pore area scaling and rotation and (e) subtraction of the pore area from a solid unit RVE

Figure 2.A.2: Steps for 3D modeling of a 3D Shellular RVE: (a) points and curves satisfying the level surface equation, (b) smooth surface passing through the points and curves to create the fundamental region, (c) using transformation to create the unit cell from the fundamental region and (d) adding thickness to the middle surface.

Figure 2.A.3: Obtained effective thermal conductivities based on the discussed numerical homogenization scheme (solid lines) and the values reported in [20] for the same periodic RVEs

Figure 2.A.4: Relation between effective principal thermal conductivities of thin-walled open lattices for different $m$ and $n$ values of porous faces and $0^{\circ} \leq \beta \leq 90^{\circ}$...... 45

Figure 3.1: Fundamental regions of (a) Primitive, (b) Diamond, and (c) Gyroid TPMS 49

Figure 3.2: Effect of increasing the number of divisions on the smaller edge of the rectangular mapping of the fundamental region of G-type TPMS on the cell aspect ratio, while the longer edge is being divided by the nearest integer

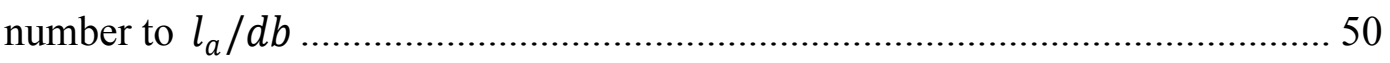

Figure 3.3: Steps for CAD modeling of conformally perforated TPMS............................ 51

Figure 3.4: Sample FDM 3D printed CPSMs based on layouts of 2-by-2 2D cells of 0.5 relative density: (a) P-type CPSM with shifted semi-circular pores, (b) D-type 
CPSM with semi-square pores and (c) G-type CPSM with semi-circular pores.

Figure 3.5: Representative CAD models of CPSMs based on: (a) P-type Shellular and 2D rectangular cell with an elliptic pore, (b) P-type Shellular and 2D reentrant square cell, (c) D-type Shellular and 2D square cell with shifted circular pore, and (d) G-type Shellular and 2D rectangular cell with an arbitrary pore shape. Relative thicknesses of all models are 0.02 . 54

Figure 3.6: Dependency of properties of the selected CPSMs (described in Table 3.1) on the number of mapped holes on the fundamental region of the underlying Psurface, (a) normalized effective thermal conductivity and (b) relative density. Effective thermal conductivities denoted with a star are normalized by the conductivity of the base material 56

Figure 3.7: (a) Overall thermal conductivity of P-type CPSMs (with 0.02 relative thickness) with respect to relative density of its underlying 2D architectures; (b) and (c) show the RVEs of the P-type CPSM with semi-circular and semisquare holes with $2 \mathrm{D}$ relative densities equal to 0.5 .

Figure 4.1: (a) FCC and (b) SC truss-like architectures at the nano and macro scales. 61

Figure 4.2: Effective thermal conductivity of SC and FCC Diamond trusses, (a) with respect to $\mathrm{N}$, and (b) with respect to density

Figure 4.3: Effective mechanical properties of SC and FCC Diamond trusses along coordinate axes, (a) with respect to $\mathrm{N}$, and (b) with respect to density 65

Figure 4.4: Specific effective moduli of FCC and SC diamond truss architectures with respect to their effective thermal conductivities. 66

Figure 4.5: Effective properties of SC and FCC truss architecures at the macro and nano scale as a function of relative density, (a) Effective thermal conductivity, (b) Effective specific modulus along $\mathrm{x}$ axis ([100] direction), and (c) Effective Specific shear modulus ([110] direction)

Figure 4.6: Specific modulus ([100] direction) of nano and macro scale SC and FCC trusses with respect to effective thermal conductivity compared to other materials (as reported in [23]) 


\section{LIST OF TABLES}

Table 2.1: $\mathrm{A}_{0}$ for different $\mathrm{n}$ values

Table 2.2: Closed-form expressions for $K_{y y}^{e f f} / K_{m}$ of cells made by thermally isotropic and homogeneous matrix and filler material (inclusion), having rectangular and elliptic inclusion/pore shapes, under the assumptions of 'Horizontal isothermal lines' or 'Vertical adiabatic lines'

Table 2.3: Thermal response of a square cellular part made by an anisotropic cellular architecture, subjected to a negative temperature gradient in the $\mathrm{x}$ direction $\left(T_{l}>T_{r}\right)$ and different boundary conditions in the y direction. Thick solid black lines represent insulated walls.

Table 2.4: Curve fittings of the normalized effective thermal conductivity of $P, D$ and $G$ Shellular materials in the form of $K^{\text {eff }} / K_{m}=C_{0}\left(\rho_{r}\right)^{n}$

Table 2.5: Temperature contours of architected and graded cellular media along with the minimum and maximum values for $\mathrm{Q}_{\mathrm{r}}, \mathrm{Q}_{t}$ and $\mathrm{T}_{\max }$. The results of a solid medium are also provided as a baseline for comparison.

Table 3.1: Characteristics of the underlying architectures of the selected P-type CPSM....... 55

Table 4.1: Relative dimensions of the selected nanotruss architectures 62

Table 4.2: Relative densities of the simplified macroscopic Diamond-truss architectures..... 62

Table 4.3: Material properties for Diamond [72] 63 


\section{LIST OF SYMbOLS}

$a$

$a 1, a 2, a 3$

A

$A_{0}$

$b$

$b 1, b 2, b 3$

c

$c 1, c 2, c 3$

$C_{i j m n}$

$\bar{C}_{i j k l}$

$D_{\text {junction }}$

$D_{\text {tube }}$

$D_{r}^{\text {junction }}$
- In Chapter 2: Pore length along x direction

- In Chapter 3: longer edge of the rectangle resulted from conformally mapping the fundamental region of the TPMS

Effective 2D thermal conductivities of zx, yz and xy faces of a general thinwalled open lattice, along $\mathrm{x}$ direction of their respective local coordinate systems

Parameter for estimating the effective thermal conductivity of RVEs with elliptic inclusions, along y direction, based on vertical adiabatic lines assumption

Shape parameter introduced to the modified superformula in order to consider relative density or the supershape RVE

- In Chapter 2: Pore width along y direction

- In Chapter 3: shorter edge of the rectangle resulted from conformally mapping the fundamental region of the TPMS

Effective 2D thermal conductivities of $\mathrm{zx}, \mathrm{yz}$ and $\mathrm{xy}$ faces of a general thinwalled open lattice, along y direction of their respective local coordinate system

Average effective principal thermal conductivity of $2 \mathrm{D}$ cellular materials $\left(=\left(K_{1}+K_{2}\right) / 2\right)$

Off-diagonal terms of Effective 2D thermal conductivities of zx, yz and xy faces of a general thin-walled open lattice, in their respective local coordinate system

Local stiffness tensor inside the RVE

Effective stiffness tensor

Diameter of the junctions of the CNT-based nanotruss (mid thickness)

Diameter of the CNT (mid thickness)

Relative diameter of the junctions with respect to the length of the RVE in a CNT-based nanotruss RVE 


\begin{tabular}{|c|c|}
\hline$D_{r}^{\text {tube }}$ & $\begin{array}{l}\text { Relative diameter of the CNT with respect to the length of the nanotruss } \\
\text { RVE }\end{array}$ \\
\hline$E$ & Young's modulus \\
\hline$E_{e f f}$ & Effective Young's modulus \\
\hline$F$ & Shape parameter in the approximate level surface equations of TPMSs \\
\hline$G$ & Shear modulus \\
\hline$G_{e f f}$ & Effective shear modulus \\
\hline$G_{x z}, G_{y z}, G_{x y}$ & shear moduli along the main coordinate axis \\
\hline$H$ & Heat convection coefficient \\
\hline$I$ & Identity tensor \\
\hline K & Thermal conductivity \\
\hline$K_{1}$ & Maximum principal thermal conductivity \\
\hline$K_{1}^{e f f}$ & Maximum effective principal thermal conductivity \\
\hline$K_{2}$ & Minimum principal thermal conductivity \\
\hline$K_{2}^{e f f}$ & Minimum effective principal thermal conductivity \\
\hline$K_{3}$ & Principal thermal conductivity (between $K_{2}$ and $K_{1}$ ) \\
\hline$K^{e f f}$ & Effective thermal conductivity \\
\hline$K_{\text {air }}$ & Thermal conductivity of the air $(=0.0263 \mathrm{~W} / \mathrm{mK})$ \\
\hline$K_{C P S M}^{e f f}$ & $\begin{array}{l}\text { Effective thermal conductivity the CPSM. If denoted with a star, it is } \\
\text { normalized by the conductivity of the base material }\end{array}$ \\
\hline$K_{i}$ & $\begin{array}{l}\text { Thermal conductivity of the inclusion material, assumed to be smaller than } \\
\text { that of matrix material }\end{array}$ \\
\hline$K_{i k}$ & Local thermal conductivity tensor inside the RVE \\
\hline $\bar{K}_{i j}$ & Effective thermal conductivity tensor \\
\hline$K_{m}$ & Thermal conductivity of the matrix material \\
\hline$K_{\text {nanotruss }}$ & Effective thermal conductivity of the CNT-based nanotruss \\
\hline$K_{\text {Shellular }}^{\text {eff }}$ & $\begin{array}{l}\text { Effective thermal conductivity of the underlying } 3 \mathrm{D} \text { shellular architecture } \\
\text { of the CPSM. If denoted with a star, it is normalized by the conductivity of } \\
\text { the base material }\end{array}$ \\
\hline$K_{x x}, K_{y y}, K_{z z}$ & Thermal conductivity along $\mathrm{x}, \mathrm{y}$ and $\mathrm{z}$ directions respectively \\
\hline
\end{tabular}


$K_{x x}^{e f f}, K_{y y}^{e f f}, K_{z z}^{e f f} \quad$ Effective thermal conductivity along $\mathrm{x}, \mathrm{y}$ and $\mathrm{z}$ directions respectively

$K_{x y}, K_{x z}, K_{y z} \quad$ Off-diagonal term of the thermal conductivity tensor

$K_{x y}^{e f f}, K_{x z}^{e f f}, K_{y z}^{e f f} \quad$ Off-diagonal term of the effective thermal conductivity tensor

$\begin{array}{ll}K_{x y f a c e}^{e f f}, K_{z x f a c e}^{e f f} & \text { Effective in-plane thermal conductivities of the faces of a general 3D thin- } \\ K_{y z f a c e}^{e f f} & \text { walled open lattice in their corresponding local 2D coordinate systems } \\ K_{2 D}^{e f f} & \text { Effective thermal conductivity of the underlying 2D architecture of the }\end{array}$ CPSM. If denoted with a star, it is normalized by the conductivity of the base material

$K_{3 D}^{e f f}$
$l, L_{R V E}$
$l_{a}, l_{b}$
$l_{x}, l_{y}, l_{z}$
$m$

Effective thermal conductivity tensor of a 3D thin-walled open lattice

Dimension of the square/cubic RVE

Lengths of the longer and shorter edges of the rectangle, resulted from conformally mapping the fundamental region of the TPMS

RVE's dimensions along the $x, y$ and $z$ axis respectively

Shape parameter in the modified Superformula, which defines the pore order of rotational symmetry

$M^{T} \quad$ Tensor which relates the average and local temperature gradients in an RVE by $\nabla T=M^{T} \overline{\nabla T}$

$M^{C} \quad$ Tensor which relates the average and local strains in an RVE by $\varepsilon_{i j}=$ $M_{i j k l}^{C} \bar{\varepsilon}_{k l}$

n

- In Chapter 2: Shape parameter in the modified Superformula

- In Chapter3: Number of 2D cells mapped on the fundamental region of the TPMS (in a $n \times n$ layout)

$n_{x}, n_{y} \quad$ Number of cells in the $\mathrm{x}$ and $\mathrm{y}$ directions of the FGCM part, respectively

$\mathrm{N}$

Tube length parameter used in the MD simulation of the CNT-based nanotrusses

$\overrightarrow{\mathrm{q}} \quad$ Heat flux vector

q Heat flux magnitude

$q_{l}, q_{r}, q_{t}, q_{b} \quad$ Heat fluxes passing through the left, right, top and bottom walls of the 2D part respectively 


\begin{tabular}{|c|c|}
\hline$Q_{r}, Q_{t}, Q_{b}$ & $\begin{array}{l}\text { Heat flow passing through the right, top and bottom edges of the } 2 \text { D FGCM } \\
\text { part }\end{array}$ \\
\hline$Q_{\text {in }}$ & Inlet heat flow through the left side of the 2D FGCM part \\
\hline$R$ & $\begin{array}{l}\text { Radius of the Mohr's circle used in determining thermal conductivity of } \\
\text { rotated } 2 \mathrm{D} \text { materials }\left(=\left(K_{1}-K_{2}\right) / 2\right)\end{array}$ \\
\hline $\mathrm{R}^{3}$ & Standard three dimensional coordinate system \\
\hline$R^{y}$ & Thermal resistance along y direction \\
\hline$\delta R^{y}$ & Differential thermal resistance element along y direction \\
\hline$\delta R_{m}^{y}$ & $\begin{array}{l}\text { Differential thermal resistance element along y direction caused by matrix } \\
\text { material }\end{array}$ \\
\hline$\delta R_{i}^{y}$ & $\begin{array}{l}\text { Differential thermal resistance element along y direction caused by } \\
\text { inclusion material }\end{array}$ \\
\hline$S$ & Scaling factor along $\mathrm{x}$ direction in the modified superformula \\
\hline$T$ & $\begin{array}{l}\text { Wall thickness. In Chapter } 4 \text { it represents thickness of the CNT, equal to } \\
\text { Graphene's layer thickness }\end{array}$ \\
\hline$t_{r}$ & $\begin{array}{l}\text { Relative wall thickness, equals to the thickness of the walls/shells inside the } \\
\text { cellular material divided by RVE's length }\end{array}$ \\
\hline$T(x, y, z)$ & Temperature at a point located at $(\mathrm{x}, \mathrm{y}, \mathrm{z})$ location \\
\hline$T_{l}, T_{r}, T_{t}, T_{b}$ & Temperatures of left, right, top and bottom walls respectively \\
\hline$T_{\max }$ & Maximum temperature throughout the FGCM part \\
\hline$T_{\infty}$ & Ambient temperature \\
\hline$\vec{\nabla} \mathrm{T}$ & Temperature gradient \\
\hline$\overline{\nabla T}$ & Average temperature gradient \\
\hline$\frac{\partial T}{\partial x}, \frac{\partial T}{\partial y}, \frac{\partial T}{\partial z}$ & Temperature gradients along $\mathrm{x}, \mathrm{y}$ and $\mathrm{z}$ directions respectively \\
\hline$\frac{\overline{\partial T}}{\partial x}, \frac{\overline{\partial T}}{\partial y}, \frac{\overline{\partial T}}{\partial z}$ & Average temperature gradients along $\mathrm{x}, \mathrm{y}$ and $\mathrm{z}$ directions respectively \\
\hline$V_{R V E}$ & Volume of the RVE \\
\hline$x_{0}$ & $\mathrm{x}$ coordinate of the face of the cubic RVE with $-\mathrm{x}$ axis as its normal direction \\
\hline$x^{\prime}$ & $\begin{array}{l}\mathrm{x} \text { coordinate of the points satisfying the modified Superformula (before } \\
\text { considering the relative density) }\end{array}$ \\
\hline
\end{tabular}




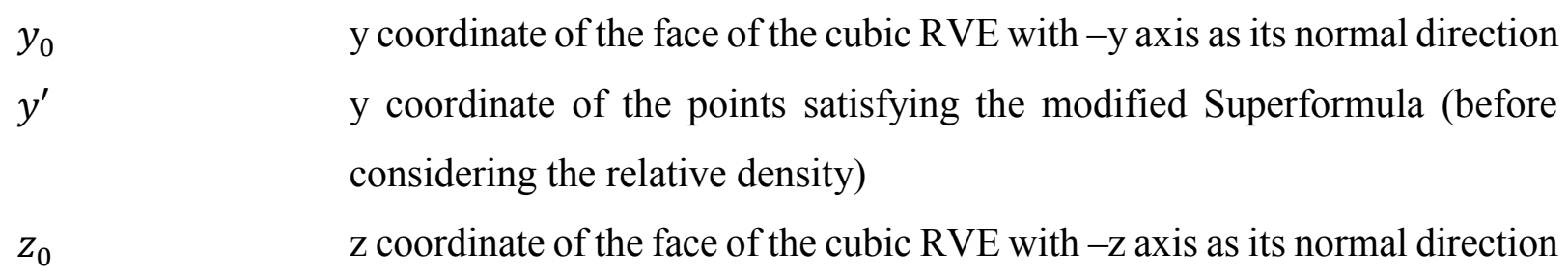

\section{Greek symbols}

$\alpha($ alpha $)$
$B$
$\beta_{1}, \beta_{2}, \beta_{3}, \beta_{4}$
$\beta(i, j)$
$\gamma$
$\varepsilon$
$\bar{\varepsilon}$
$\theta$

Angular location of the points on the boundary of the supershape pores

Rotation angle in the modified superformula. Equal to pore rotation angle in supershape RVEs

Pore rotation angle at the 4 corners of the 2D FGCM medium

Pore rotation angle of the cell located at $i^{\text {th }}$ column and $j^{\text {th }}$ row of the $2 D$ FGCM part

Integral variable for calculating the enclosed area inside the modified superformula

Local strain tensor inside the RVE

Average strain tensor inside the RVE

Parameter for estimating the effective thermal conductivity of RVEs (with elliptic inclusions) along y direction, based on horizontal iso-thermal lines assumption

$\lambda_{a} \quad$ Relative length of the pore $(=a / l)$

$\lambda_{b} \quad$ Relative width of the pore $(=b / l)$

$\lambda_{k}$

v

Ratio of the thermal conductivities of inclusion and matrix materials (= $\left.K_{i} / K_{m}\right)$

$v_{e f f}$

Poisson's ratio

Effective Poisson's ratio

$\pi$

Pi number, approximately equal to 3.14159

$\left(\rho_{m}\right)_{r}$

Relative density of the matrix

$\rho_{r}$

Relative density, equals to 1- $\Phi$

$\rho_{r}^{2 D}$

Relative density of the underlying 2D architecture of the CPSM

$\rho_{r}^{\text {Shellular }}$

Relative density of the underlying 3D shellular architecture of the CPSM 
Porosity of the cellular material, defined as the pore area divided by the whole cell area for 2D RVEs, and pore volume divided by the volume of the RVE for 3D RVEs 


\section{List of AbBreviations}

2D Two dimensional

3D Three dimensional

APDL ANSYS parametric design language

atan Inverse tangent function

BCC Body-centered cubic

CAD Computer-aided design

CNT Carbon nanotube

Cos Cosine function

CPSM Conformally perforrated shellular materials

CREPEC Center for high performance polymer and composite systems

D Schwarz's Diamond surface

Eq. Equation

FCC Face-centered cubic

FDM Fused deposition modelling

FEM Finite element method

FGCM Functionally graded cellular material

Fig. $\quad$ Figure

G Schoen's Gyroid surface

laser Light amplification by stimulated emission of radiation

Ln Natural logarithm

Log Base 10 logarithm

max Maximum

MD Molecular dynamics simulation

min Minimum

NSERC Natural sciences and engineering research council of Canada

P Schwarz's Primitive surface

PLA Polylactic acid

RVE Representative volume element

SC Simple cubic 
SI International System of Units

Sin Sine function

SLA Stereolithography or resin printing

SLS Selective laser sintering

TPMS Triply periodic minimal surface

\section{Units}

C Centigrade or Celsius scale - temperature scale used in SI

$\mathrm{cm} \quad$ centimeter - equal to $10^{-2}$ meter

$\mathrm{GPa} \quad$ Giga Pascal - equivalent to $10^{9}$ pascal

$\mathrm{K} \quad$ kelvin - base unit of temperature in SI

$\mathrm{kg} \quad$ kilogram - base unit of mass in SI

$\mathrm{m} \quad$ meter - unit of length in SI

$\mathrm{mm} \quad$ millimeter - equal to $10^{-3}$ meter

MN mega newton - equivalent to $10^{6}$ newton

$\mathrm{N} \quad$ Newton - unit of force in SI - the force needed to accelerate one kilogram of mass at the rate of one meter per second squared

$\mathrm{Pa} \quad$ Pascal - pressure unit in SI, the equivalent of one newton per square meter

W Watt - standard unit of power (energy per unit time) in SI, the equivalent of one joule per second 


\section{Abstract}

Bio-inspired cellular materials have attracted considerable attention in the past few decades. Low thermal conductivity, high impact absorbance, wide range of porosity and permeability, sound and vibration insulation, and lots of other interesting properties all at lower densities compared to regular solid materials like metals, make these materials worthy of a closer investigation. As a well-known example, wood with its outstanding properties is a cellular structure, which is the result of millions of years of evolution that has perfected the building blocks of plants. Understanding the underlying physical phenomenon responsible for the properties of cellular materials will pave the way towards designing new materials with unprecedented properties. A widely used artificial cellular architecture, especially in aviation industries, is the honeycomb core of the sandwich composites, which is lightweight and stiff, with in-plane isotropic properties and low thermal conductivity.

With a similar inspiration, this thesis focuses on the thermal conductivity of $2 \mathrm{D}$ and $3 \mathrm{D}$ cellular metamaterials. With the help of CAD packages like Solidworks and ANSYS, different cell architectures are modeled and their effective thermal conductivities are obtained by adopting standard mechanics homogenization technique on a representative cell under periodic boundary conditions. A wide variety of 2D pore shapes based on a modified form of Gielis' superformula is analyzed to inspect the effect of different topological parameters. Furthermore, a case study in 2D is provided to explore how the concept of functionally graded cellular materials can be used to tune the heat flow and temperature within a part made of cellular materials.

3D thin-walled open lattices are also introduced based on the 2D cells with supershape pores. Effective thermal conductivity of these 3D architectures are compared with those of Shellular materials. Discrete conformal mapping is employed to introduce holes in Shellular architectures, making it possible to adjust effective properties of the Shellular materials. While the relative density of a conformally-perforated Shellular material (CPSM) is less than its Shellular counterpart, the existence of holes further increases the overall permeability of this new architected metamaterial. Analysis of the correlation between the effective thermal conductivities of the underlying 2D and 3D architectures of the P-type CPSM shows the effect of using cellular structure at different length scales in designing a new ultralight metamaterial. Effective thermal conductivities of CPSM are studied under the assumption that same physics are applicable at the 
involved length scales, in other words, underlying 2D and 3D cellular architectures are both at continuum level and Fourier heat conduction can be used to accurately model heat conduction through these materials. On the other hand, for structures at the nanoscale, different physics are involved in heat transfer and sophisticated methods such as Molecular Dynamics simulation are mostly used to study their properties. To show the length scale dependency of effective properties, macroscopic counterparts of some nano-architected metamaterials with face centered cubic (FCC) and simple cubic (SC) arrangements, designed based on carbon nanotube (CNT) nanotrusses, are created and their effective properties are obtained using standard mechanics homogenization. 


\section{RÉSUMÉ}

Les matériaux cellulaires bio-inspirés ont attiré une attention considérable au cours des dernières décennies. Une faible conductivité thermique, une forte absorbance aux chocs, une large gamme de porosité et de perméabilité, une isolation vibratoire et acoustique et de nombreuses autres propriétés intéressantes, toutes à des densités inférieures à celles de matériaux solides normaux tels que les métaux, rendent ces matériaux dignes d'une recherche plus approfondie. En tant qu'exemple bien connu, le bois, avec ses propriétés exceptionnelles, est une structure cellulaire, produit de millions d'années d'évolution qui ont perfectionné les éléments constitutifs des plantes. La capacité de comprendre le phénomène physique responsable pour les propriétés avancées des matériaux cellulaires permettra la conception de nouveaux matériaux aux propriétés sans précédent. Une structure cellulaire artificielle largement utilisée, en particulier dans les industries de l'aviation, est l'âme en nid d'abeilles des composites sandwich, qui est léger et rigide, avec des propriétés isotropes dans le plan et une faible conductivité thermique.

En suivant une inspiration similaire, cette thèse porte sur la conductivité thermique des métamatériaux cellulaires 2D et 3D. À l'aide de logiciels de CAD tels que Solidworks et ANSYS, différentes architectures de cellules sont modélisées et leurs conductivités thermiques effectives sont obtenues en adoptant la technique d'homogénéisation mécanique classique sur une cellule représentative dans des conditions aux limites périodiques. Une grande variété de formes de pores 2D basées sur une version modifiée de la superformula de Gielis est analysée pour examiner l'effet de différents paramètres topologiques. De plus, une étude de cas en 2D est fournie pour explorer comment le concept de matériaux cellulaires à gradations fonctionnelles peut être utilisé pour régler le flux de chaleur et la température dans une pièce constituée de matériaux cellulaires.

Des réseaux $3 \mathrm{D}$ à parois minces sont également introduits sur la base des cellules $2 \mathrm{D}$ à pores supershape. La conductivité thermique effective de ces architectures 3D est comparée à celle des matériaux Shellular. La mise en correspondance discrète est utilisée pour introduire des trous dans les architectures Shellular, ce qui permet d'ajuster les propriétés effectives des matériaux Shellular. Alors que la densité relative d'un matériau Shellular perforé de manière conforme (CPSM) est inférieure à son équivalent Shellular, la présence de trous augmente davantage la perméabilité globale de ce nouveau métamatériau architecturé. L'analyse de la corrélation entre les conductivités thermiques effectives des architectures 2D et 3D sous-jacentes du CPSM de type P 
montre l'effet de l'utilisation d'une structure cellulaire à différentes échelles de longueur dans la conception d'un nouveau métamatériau ultra-léger. Les conductivités thermiques effectives du CPSM sont étudiées en supposant que la même physique est applicable aux échelles de longueur impliquées, autrement dit, les architectures cellulaires $2 \mathrm{D}$ et $3 \mathrm{D}$ sous-jacentes sont à la fois au niveau du continuum, et la conduction thermique de Fourier peut être utilisée pour modéliser avec précision la conduction thermique à travers ces matériaux. D'autre part, pour les structures à l'échelle nanométrique, le transfert de chaleur implique différents phénomènes physiques et des méthodes sophistiquées telles que la simulation par dynamique moléculaire sont principalement utilisées pour étudier leurs propriétés. Pour montrer la dépendance des propriétés effectives sur l'échelle de longueur, des équivalents macroscopiques de certains métamatériaux nanoarchitecturés avec des structures cubiques à faces centrées (FCC) et cubiques simples (SC), conçus à partir d'une armature nanométrique de nanotubes de carbone (CNT), sont créés et leurs propriétés effectives sont obtenues à l'aide de l'homogénéisation mécanique classique. 


\section{ACKNOWLEDGEMENTS}

I would like to express my sincere gratitude to my supervisor, Prof. Abdolhamid Akbarzadeh, for his nice personality, his exemplary patience, his support and for providing guidance on all aspects of this work, from scientific details to aesthetics. Thank you for accepting and trusting me.

I am eternally grateful to McGill University, to the department of Bioresource engineering, and to the governments of Quebec and Canada for giving me the opportunity to live and study here.

I would also like to specially thank my family, Commander-in-Chief my lovely mom, my brilliant always-supportive sister Prof. Maryam, and my cheerful polymath brother Dr. Pejman. Without their sacrifices and selflessness, it could have never been possible for me to do an MSc in Canada.

Finally, I would like to thank all $\mathrm{AM}^{3} \mathrm{~L}$ lab members, Hamed, Shahin, Liang, Jiahao, Niousha, Hamidreza, Zhonghua, Chunyi, Minghan, Nicholas and Alain, whom I learnt a lot from during the past two years and shared unforgettable memories. Especial thanks to Nicholas for his help with French translation of the abstract. 


\section{Preface And Contribution of Authors}

In accordance with the McGill University guidelines for thesis preparation ${ }^{1}$, the present thesis is written in the article-based style by Armin Mirabolghasemi under the supervision of Prof. Abdolhamid Akbarzadeh, in the department of Bioresource Engineering at the beautiful Macdonald campus of McGill University.

At Advanced Multifunctional Multiphysics Metamaterials Laboratory $\left(\mathrm{AM}^{3} \mathrm{~L}\right)$ of McGill University, different properties and applications of architected cellular metamaterials are being investigated through numerical simulations and experimental analysis of 3D printed parts. While these studies are mostly focused on mechanical properties, Armin's research has centered on introducing novel architected cellular metamaterials and investigating their thermal properties, which are reported in the following publications and constitute the majority of this thesis.

\section{Journal articles}

- A. Mirabolghasemi, A.H. Akbarzadeh, D. Rodrigue, D. Therriault, "Thermal conductivity of architected cellular metamaterials", 2018 (under review)

- C. Zhang, A.H. Akbarzadeh, W. Kang, J. Wang, A. Mirabolghasemi, "Nano-architected metamaterials: Carbon nanotube-based nanotrusses", Carbon, 2018. 131: p. 38-46

\section{Conference papers}

- A. Mirabolghasemi, A.H. Akbarzadeh, D. Rodrigue, D. Therriault, "Thermal conductivity of advanced architected cellular materials", Proceedings of 2018 CSME International Congress, Toronto, ON, Canada, May 2018

- C. Zhang, A.H. Akbarzadeh, W. Kang, J. Wang, A. Mirabolghasemi, "CNT-based architected metamaterials: Thermo-mechanical characteristics", APS March Meeting 2018, Los Angeles, CA, USA, March 2018

For the first journal publication listed above as the main part of this thesis, the research, including literature review, CAD modeling, theoretical and numerical simulations, analysis of the result and writing, is conducted by Armin under direct supervision of Prof. Akbarzadeh, who provided scientific suggestions in the development of the work and helped polishing the written

\footnotetext{
${ }^{1}$ https://www.mcgill.ca/gps/thesis/thesis-guidelines/preparation
} 
manuscript. Prof. Rodrigue and Prof. Therriault also contributed to the conception of the work and were involved in technical editing of the manuscript.

For the second journal publication, Armin has performed CAD modeling and conducted numerical homogenization to investigate the effective thermal conductivity and mechanical properties of the macroscale counterparts of the CNT based nanotruss architectures. He has also contributed to data analysis and interpretation of results, by comparing the properties of the aforementioned macroscale cellular architectures with those of CNT based nanotrusses. Chunyi Zhang, the first author, has performed the studies at nanoscale and did the result analysis, under the supervisory of Prof. Akbarzadeh, Prof. Kang and Prof. Wang. Detailed description of Armin's collaboration in this article is presented in Chapter 4 of this thesis. ${ }^{2}$

Armin's contribution to two ongoing studies on the properties of Shellular and perforatedShellular materials, in collaboration with Shahin Eskandari and Jiahao Shi and under Prof. Akbarzadeh's guidance, is also presented in this thesis which includes designing and preparation of required CAD models of architectures and analysis of their effective thermal conductivities, while Shahin focuses on investigating dynamic behaviours of these materials and Jiahao performs nonlinear structural analysis to study these architectures under large deformation.

In addition to his main research topic, Armin has also contributed to the investigation of mechanical properties of architected cellular metamaterials in the following publications:

\section{Journal articles}

- H. Yazdani Sarvestani, A. H. Akbarzadeh, A. Mirabolghasemi, K. Hermenean, “3D printed meta-sandwich structures: Failure mechanism, energy absorption and multi-hit capability”, Materials \& Design, 2018. 160: p. 179-193

- H. Yazdani Sarvestani, A.H. Akbarzadeh, A. Mirabolghasemi, "Structural Analysis of Size-Dependent Functionally Graded Doubly-Curved Panels with Engineered Microarchitectures”, Acta Mechanica, 2018. 229: p. 2675-2701

\section{Conference papers}

\footnotetext{
${ }^{2}$ Permission has been granted from the publisher (Elsevier) to use materials from this article in the thesis.
} 
- H. Niknam, A.H. Akbarzadeh, A. Mirabolghasemi, D. Rodrigue, D. Therriault, "Engineered architected materials: Multidirectional functionally graded cellular solids", Proceedings of $14^{\text {th }}$ U.S. National Congress on Computational Mechanics, Montreal, QC, Canada, July 2017

- H. Yazdani Sarvestani, A.H. Akbarzadeh, A. Mirabolghasemi, S. Rankohi, "Engineered Graded Materials: Vibration of Doubly-Curved NanoPanels", CSCE Annual Conference, Vancouver, BC, Canada, May 2017 


\section{LITERATURE REVIEW AND GENERAL INTRODUCTION}

Cellular solids, a class of material that consists of clusters of cells with 2D or 3D walls and gaseous or empty pores, span a wide range of natural and artificial materials. Several classes of cellular solids have been defined based on their characteristics, such as open-celled and closedcelled cellular materials, for when pores are connected or separate. While the interconnected network of struts and shells in an open-cell structure, such as open-cell sponge, makes it permeable for fluids, in a closed-cell architecture such as cork, pores are enclosed by the solid phase and the material is impermeable [1]. Closed-cell materials are generally mechanically stronger than the open-cell architectures [2]. Cellular solids are also divided to Foam and lattices, in which the former has random cell architecture and/or arrangement throughout the material, contrary to the latter which is a periodic assembly of one unit cell. Sponge and honeycomb structures are two examples of the two groups, respectively. There are also other classifications based on wall thickness, relative density or even material's application [1]. In the context of this thesis, the focus would be on architected periodic cellular materials and the word cellular only refers to the lattice structures.

Although the use of natural cellular materials, such as wood and cork, dates back thousands of years and cellular geometries such as honeycomb have fascinated many throughout the history, it was not until the $20^{\text {th }}$ century that the manmade cellular materials became widely used. Foams for thermal insulation, metallic honeycomb core sandwich plates in aircrafts, and paper honeycomb core plasterboard panels can be named as some of the early applications of cellular materials, in which they were mostly used to reduce the weight without sacrificing the strength [1, $3,4]$. With new and growing industries, ideal materials today should have multiple functionality, from strength and durability to thermal/electrical conductivities, liquid or gas permeability, shock and vibration absorbance, and so on [5, 6]. In terms of thermal applications, although cellular materials are generally considered as lightweight insulators without load bearing capabilities, recent studies have shown their promising potential in other applications such as lightweight load bearing insulations for building materials [7], thermal conductivity enhancement for thermal storage units at high temperatures [8], thermal management in microelectronics [9] and thermal shock absorbers [10]. 
In order to design cellular materials with desired thermal properties, it is important to identify factors that play a role in transferring heat through the cellular media, and employ an accurate mathematical model to investigate the involved heat transfer physical phenomena. At atomic and nano scale, phonon and electron transport and their interactions with the material's architecture at that length scale define the heat transfer [11]. In continuum level, in addition to the selfexplanatory role of constituent materials' conductivities and the rather unsurprising effect of their relative amount, pore topology (i.e. size, shape, connectivity and orientation of the pores) is shown to significantly affect the overall thermal conductivity of a cellular architecture [1]. This thesis concentrates on the thermal properties of cellular materials at the macro scale.

Rule of mixtures is probably the oldest and arguably the simplest mathematical model for estimating the overall properties of cellular materials, which only takes into account thermal conductivities of constituent materials and their relative amount. Although predictions of this simple model is surprisingly good for certain cases, they generally deviate significantly from the actual properties as a consequence of ignoring the impact of underlying architectures. Empirical models dealing with a single or a family of simple cellular architectures were developed to address the lack of accuracy of the Rule of mixtures model, though they were inapplicable for other architectures $[1,12]$. One of the concepts that enabled accounting microarchitecture for estimating the effective thermal conductivity of cellular materials is the analogy between electric and heat conduction, which was the basis of thermal circuit modeling [13, 14]. However, similar to few other concepts, using this method only provides upper and lower bounds and not the exact effective thermal conductivity. A summary of popular closed form expressions for estimating the effective thermal conductivity of heterogeneous materials and their limitations can be found in reference [15]. On the other hand, during the last few decades advanced computational methods, such as homogenization techniques [16, 17] and lattice-based Monte-Calro approach [18, 19], have been developed which are capable of accounting for the microarchitecture in predicting the effective thermal conductivities of cellular materials. With the help of the new methods, in addition to mechanical properties, investigating thermal conductivity of a wide range of cellular architectures (such as cellular solids with convex or concave pores [20], metamaterials based on Gibson-Ashby cells [21], metallic hollow spheres structures [3, 22] and even nanostructured materials [23]) has been a compelling topic among material researchers, providing insights about the applications of cellular materials in the field of thermal management such as heat exchangers and lightweight heat 
insulations. Considering the relative simplicity of implementing the numerical standard homogenization technique, effective thermal conductivities of different architectures are obtained using this method throughout this thesis, in which adopting periodic boundary conditions guarantees the accuracy of the predictions.

Although theoretical and numerical study on thermal properties of cellular materials have been an active field of research in the past few decades, studies that explore the microarchitecture are mostly focused on truss-like lattices or cellular materials with simple pore topologies such as those based on rectangle and ellipse in 2D or cuboid and ellipsoid in 3D [20, 21, 24, 25]. In the present thesis, it is tried to study a wide range of cell architecture in $2 \mathrm{D}$ and $3 \mathrm{D}$, and where possible offer alternative mathematical approaches to better understand the underlying correlation between cell microarchitecture and its effective thermal conductivity.

Contents of this thesis are organized in five chapters. After a general introduction in this chapter, Chapter 2 as the main part of this thesis constitutes the following manuscript prepared and submitted for publication:

- A. Mirabolghasemi, A.H. Akbarzadeh, D. Rodrigue, D. Therriault, "Thermal Conductivity of Architected Cellular Metamaterials”, 2018 (under review)

in which, effective thermal conductivity of several 2D and 3D architected cellular materials are extensively studied using numerical homogenization. Novel architectures are introduced to better investigate the relation between pore topology and heat conduction in 2D cellular materials. Based on these 2D cells, 3D architectures are constructed and their homogenized thermal conductivity is obtained. A mathematical model is also developed to further examine correlation of the thermal conductivity of the 3D architectures and their underlying 2D cells. Moreover, selected Shellular materials are also compared with the aforementioned 3D cellular materials. A case study is also provided to demonstrate the potential use of functionally graded cellular materials to guide heat through a porous medium.

Chapter 3 addresses some shortcomings of macroscopic Shellular architectures by perforating them with a mapped 2D layout of pores in regular cellular arrangements. Conformal mapping is employed to minimize the area distortion through the mapping process and preserve cell topology as much as possible. It is shown that although $2 \mathrm{D}$ cell architecture is not preserved through the 
mapping, with enough number of pores effective thermal conductivity of this doubly porous cellular material can be precisely determined based on the overall thermal conductivities of the underlying $2 \mathrm{D}$ and $3 \mathrm{D}$ architectures. In collaboration with other researchers, mentioned in the preface section, dynamic responses and non-linear large deformation behaviour of the architectures made with the procedure discussed in this chapter are currently being studied.

In Chapter 3, it is assumed that all cell features are at continuum level, and as a result no length scale dependency is expected in the thermal behaviour of the perforated Shellular architectures. However, thermal conductivity of architectures at the nanoscale is generally different than their analogous macroscale counterpart. In Chapter 4, using the characteristic dimensions of two types of novel carbon nanotube-based nano-trusses, simplified macroscopic models are created to obtain their effective properties at continuum length scale by employing numerical homogenization on a representative cell with periodic boundary conditions. Calculated effective properties are used to show the aforementioned length dependency of thermal properties of selected truss-like cellular architectures. Chapter 4 is based on the contribution of the author of this thesis that have been appeared in the following journal publication:

- C. Zhang, A.H. Akbarzadeh, W. Kang, J. Wang, A. Mirabolghasemi, "Nano-architected metamaterials: Carbon nanotube-based nanotrusses", Carbon, 2018. 131: p. 38-46

Finally, in Chapter 5 concluding remarks and suggested future works are provided.

\section{MAJOR ASSUMPTIONS}

- A cellular material is assumed to be an assembly of perfectly periodic cells, without imperfections, and with architectural features at continuum length scale (unless otherwise stated)

- Following some criteria found in the literature, contributions of convection and radiation to overall heat transfer through the cellular media are assumed small and neglected. In addition, analysis of square cells with air inside their square pores, shows that for this architecture, air inside the pore can be ignored if thermal conductivity of the base solid material is several orders of magnitude larger than air, such as the case for metallic base cellular materials. For this assumption to be acceptable at relative densities below 0.1 , the cellular material shall be made 
from a highly conductive metal or technical ceramic. Although this analysis is done only on 2D square cells with square pores, for the rest of the manuscript, it is assumed that either the thermal conductivity of the gas inside the pores is negligible (such as poorly conductive gases at low pressures) or the pores are completely empty.

\section{RESEARCH OBJECTIVES}

The objectives of this thesis can be summarized as:

- To thoroughly analyze the effects of cell relative density and pore geometrical features (such as one directional scaling and rotation angle) on the effective thermal conductivity of 2D square cells with single confined pore with different topologies

- To investigate homogenized thermal conductivity of 3D cellular architected metamaterials

- To showcase the possibility of heat transfer manipulation inside a cellular medium using the concept of functionally graded cellular materials

- To introduce novel ultralight periodic cellular architectures and exploring their effective conductivities

- To demonstrate manufacturability of cellular architectures by means of additive manufacturing 


\section{CONNECTING Statement}

In Chapter 1, following a concise literature review, general scope and the framework of the thesis together with the objectives of the studies are presented. In Chapter 2, which constitutes the following article:

- A. Mirabolghasemi, A.H. Akbarzadeh, D. Rodrigue, D. Therriault, "Thermal Conductivity of Architected Cellular Metamaterials", 2018 (under review)

the effects of cell architecture on the effective thermal conductivity of cellular materials are thoroughly investigated by introducing novel 2D and 3D cellular architectures and implementing numerical standard homogenization. Findings are thoroughly discussed using a mathematical model and a case study, to provide better understanding of design aspects and potential use of cellular materials for thermal applications. 


\section{Thermal Conductivity of ArChitected Cellular Metamaterials}

Abstract

Periodic architected cellular materials, as a novel class of low-density materials, possess unprecedented multifunctional properties mainly due to their underlying microarchitecture. In this paper, we study the thermal conductivity of cellular metamaterials and evaluate their performance for thermal management applications. To understand the relations between the microarchitecture and the thermal response, we analyze the thermal conductivity of a wide range of cellular metamaterials with strategically developed microarchitectures from two-dimensional (2D) cells with supershape pores to three-dimensional (3D) thin-walled open lattices and Shellular materials. We implement standard mechanics homogenization on the periodic representative volume elements (RVEs) of these cellular metamaterials to examine the effect of pore architecture (relative density, pore shape, pore orientation and pore elongation) on their effective thermal conductivity. The numerical results show how the thermal conductivity of an isotropic material can be modified by pore introduction and how the pore architecture could lead to an anisotropic effective thermal conductivity tensor. To examine the impact of having 2D supershape cuts on 3D RVEs, thinwalled open lattices are designed as an assembly of thickened 2D supershape RVEs. A mathematical model is derived based on the effective thermal properties of the constituent 2D RVEs to predict the effective thermal properties of these lightweight cellular materials. Effective thermal conductivity these 3D cellular architectures are also compared with those of Shellular materials based on triply periodic minimal surfaces. Unlike the Shellular materials, which only cover a narrow region of thermal conductivity versus relative density chart, cellular materials with a wide range of anisotropic effective thermal conductivities can be engineered by using 2D supershape pores on 2D or 3D thin-walled cells. Finally, we show how the concept of architected functionally graded cellular materials can be used to tune the heat flow within cellular media guiding it in a specific direction to control the temperature inside advanced 3D printed materials. As a case study, the optimum spatial distribution of pore rotation angle is found to maximize or minimize the heat flow passing through different sides of a square-shaped porous slab.

Keywords - Architected cellular metamaterials, Effective thermal conductivity, Homogenization, Supershape pore, Thin-walled open lattice, Shellular materials, Functionally graded cellular materials. 


\subsection{INTRODUCTION}

Developing lightweight and structurally robust advanced materials with unprecedented multifunctional properties has been one of the main engineering challenges during the past few decades. Limited material and energy resources, economical restrictions and concerns over the prospect of global climate changes promote the design and manufacturing of durable lightweight materials with tunable multifunctional properties. The engineered materials with properties not available in natural materials are called metamaterials, among which bio-inspired architected cellular materials [26] are one of the cutting-edge lightweight and optimized materials that can simultaneously satisfy multiple functionalities from structural stiffness to thermal insulation or heat exchanging [5].

Cellular materials are divided into two categories: (1) Foams where a gaseous phase is randomly dispersed in a continuous solid medium, and (2) Periodic porous materials (lattices) which consist of a periodic architected cell [1]. Inspired by their excellent lightweight and mechanical properties, natural materials like bone, wood and cork led to the development of cellular materials as early as 1970 . Hexagonal honeycombs as sandwich cores, used in aviation industry, are one of the first applications of periodic cellular metals [3]. Driven by the high performance of cellular materials and recent developments in advanced manufacturing techniques such as additive manufacturing (3D printing) and laser cutting, advanced polymeric and metallic cellular materials of complex nano/microarchitectures can be designed and fabricated. A nonexhaustive list of applications of cellular materials found in the literature includes: lightweight structural elements in aircrafts and high-speed trains, energy-absorbing elements in automotive industry, thermal insulators, thermal energy storage devices, hydrogen storage tanks, and biomedical scaffolds for tissue engineering [1, 3, 8, 18, 27-32].

To exploit the multifunctional potential of cellular materials, a mathematical model capable of accurately predicting their effective properties is of crucial importance. Early efforts on this subject range from the simple volumetric averaging of properties of constituent materials, the socalled rule of mixtures method, to several empirical equations to predict the physical properties of cellular materials $[1,12]$. Investigations have clearly shown that cell microarchitecture, in addition to the constituent materials properties, plays a significant role in the emergence of the outstanding properties of cellular materials, such as negative Poisson's ratio in auxetic materials [33], as well 
as thermal and vibration insulation properties of ultralight metallic microlattices [34]. In this regard, since thermal conductivity, electrical conductivity and magnetic permeability are all mathematically described by Laplace equation, existing approaches used in the electric and magnetic fields have also been applied to predict the thermal properties of cellular materials [13, 14]. The introduction of the thermal-circuit method, based on the analogy between electrical and thermal conductivities, can be considered as a turning point in the theoretical modelling of thermal conductivity of cellular materials [14]. It is worth mentioning that most of the analytical models have been mainly developed for porous materials with random pore distribution [35], or for periodic cellular materials with simple pore topologies: i.e. cubic, circular, cylindrical and spherical pore shapes $[27,36]$, leaving cellular materials with complex periodic microarchitectures and potentially superior multifunctional properties unexplored. Although analytical upper and lower bounds, e.g., Hashin-Shtrikman bounds [37] or bounds obtained by the thermal-circuit method, are valuable for estimating the effective thermal conductivity with a minimum knowledge about the actual heat flow and temperature profile in cellular materials, advanced computational models, e.g., lattice-based Monte Carlo approach [19], micropolar modelling [38], as well as standard mechanics [16] and asymptotic [17] homogenization techniques have been introduced to precisely take into account the effect of microarchitecture of cellular materials to accurately predict their effective thermal properties.

Although the main focus of the literature on cellular materials has been devoted to their mechanical properties, the connection between cell microarchitecture and their effective thermal conductivity has also been the subject of compelling research over the past few decades. The analysis of the anisotropic thermal behavior of cellular metals [28], studies on thermal properties of new architected materials such as Shellular materials [39, 40], invention of thermal metamaterials to manipulate heat at the continuum level [41], and research on thermal properties of nanostructured cellular materials [23, 42, 43], are a few examples of the research in thermal analysis of architected cellular solids. However, to the best of the authors' knowledge, apart from cells with simple pore shapes, no in-depth study on the relation between topological parameters and the thermal conductivity tensor of periodic cellular materials with complex pore microarchitectures has been performed. As a result, a deeper understanding of the thermal properties of cellular materials is required to address the thermal requirements of emerging technologies such as lightweight electronics and catalysts. 
In this article, after introducing various cell architectures in Section 2.2, two methodologies for predicting the effective thermal conductivity of 2D cellular materials are discussed in Section 2.3: (1) Theoretical modelling using the thermal-circuit method [44] and (2) Computational modeling based on standard homogenization. In Section 2.4, after investigating the validity of assumptions made in previous sections, we first compare the aforementioned two methodologies to predict the effective thermal conductivity of 2D cellular materials with rectangular and elliptic pores. While the theoretical model can at best provide narrow bounds for the effective thermal conductivity of architected cellular materials with simple 2D microarchitectures, standard mechanics homogenization is used to accurately determine the thermal conductivity of cellular metamaterials with complex 2D pore topologies of supershape pores [45] (introduced in Section 2.2.1). In addition to an in-depth analysis on the effects of topological parameters (pore shape, pore scaling and pore rotation) on the effective thermal conductivity of these $2 \mathrm{D}$ architected cellular materials, a case study is conducted to highlight the importance of the off-diagonal terms of the anisotropic thermal conductivity tensor for the thermal analysis of cellular metamaterials. Thereafter, a mathematical model based on superposition is derived to predict the thermal conductivity of lightweight 3D thin-walled open lattices (systematically designed in Section 2.2.2) based on the effective thermal conductivity of 2D supershape architectures. Following the recent interests in periodic minimal surfaces in material engineering, the thermal conductivities of thinwalled metamaterials are compared with three Shellular materials which are lightweight microarchitectures based on triply periodic minimal surfaces (presented in Section 2.2.3). While the Shellular materials in this work exhibit isotropic effective thermal conductivities, the anisotropic thermal properties of thin-walled open lattices show possibilities for better use of cellular materials to optimize the thermo-mechanical performance of lightweight structures. Finally, in a case study conducted in Section 2.5, pore angle inside a 2D cellular medium is graded to optimize the heat flow passing through its different edges, showing how grading the cells throughout porous media provides new solutions for optimum thermal performance.

\subsection{CAD Design OF ARChitected Cellular Metamaterials}

To apply a computational homogenization method and explore the effects of pore topology and relative density on the effective thermal conductivity of cellular metamaterials, two- 
dimensional (2D) square representative volume elements (RVEs) with supershape pores (referred to as 2D supershape RVEs), 3D cubic thin-walled open lattices cut through by 2D supershape pores on opposite faces, and Shellular RVEs are examined.

\subsubsection{D SUPERSHAPE RVES}

As a powerful formula for creating a wide variety of pore topologies, Gielis' superformula [45], is selected and modified to generate alternative pore architectures, while scaling $(S)$ and rotation $(\beta)$ are considered to increase the possibilities of pore topologies. The boundary of this supershape pore in a Cartesian coordinate system is mathematically expressed as:

$$
\begin{aligned}
& {\left[\begin{array}{l}
x^{\prime} \\
y^{\prime}
\end{array}\right]=\left[\begin{array}{cc}
\cos \beta & -\sin \beta \\
\sin \beta & \cos \beta
\end{array}\right]\left([|\cos (m \alpha / 4)|+|\sin (m \alpha / 4)|]^{n}\left[\begin{array}{c}
S \cos (\alpha) \\
\sin (\alpha)
\end{array}\right]\right)} \\
& (-\pi \leq \alpha \leq \pi, m=1 \sim 8, n=-5 \sim 5, S=1 \sim 3.5(0.5 \text { increment }), 0 \leq \beta \leq \pi / 2)
\end{aligned}
$$

As introduced in Eq. (2.1), a pore gets elongated along the $x$ direction by increasing the scaling factor $S$, followed by a counter-clockwise rotation of $\beta$ degree. Using Eq. (2.1), the area of the pore

can be calculated by $4 S \int_{0}^{\frac{\pi}{4}}(\cos \gamma+\sin \gamma)^{2 n} d \gamma$ independent of the values of $\beta$ and $m$. When no scaling exists $(S=1), m$ defines the pore order of rotational symmetry, meaning that rotating the pore by an angle of $360 \% \mathrm{~m}$ does not change it.

To focus on 2D architected closed-cell metamaterials, one percent of the RVE's length is selected as the minimum allowable clearance between the pore and RVE's outer edges to avoid pore walls from touching the RVE's boundaries. For these 2D metamaterials, porosity $(\Phi)$ is defined as the ratio between the pore area and the whole cell area, which in the case of a unit square $\mathrm{RVE}$, is equal to the pore area. The relative density $\left(\rho_{r}\right)$ is defined as the ratio of the solid area to the whole cell area and is equal to: $1-\Phi$. For a unit square RVE with an enclosed 2D supershape pore, Eq. (2.1) can be rewritten in terms of the relative density $\left(\rho_{r}\right)$ via $\left[\begin{array}{l}x \\ y\end{array}\right]=$ $\left[\begin{array}{l}x^{\prime} \\ y^{\prime}\end{array}\right] \sqrt{\left(1-\rho_{r}\right) /\left(S A_{0}\right)}$, where $A_{0}$ for different $n$ values is reported in Table 2.1.

Table 2.1: Ao for different $n$ values.

\begin{tabular}{c|c|c|c|c|c|c|c|c|c|c|c}
\hline $\boldsymbol{n}$ & -5 & -4 & -3 & -2 & -1 & 0 & 1 & 2 & 3 & 4 & 5 \\
\hline $\boldsymbol{A}_{\boldsymbol{0}}$ & 0.527 & 0.686 & 0.933 & 1.333 & 2.000 & 3.142 & 5.142 & 8.712 & 15.187 & 27.078 & 49.140 \\
\hline
\end{tabular}


Figure 2.1 presents typical RVEs of the 2D architected cells for different topological parameters. Each pore topology, corresponding to each set of geometrical parameters introduced in Eq. (2.1), is modeled within ANSYS APDL (Fig. 2.A.1 in Appendix 2.A1) by creating keypoints and using spline curves. The area surrounded by the splines is formed, scaled, rotated and moved to the center of the RVE to be subtracted from the unit solid RVE and to create 2D supershape cellular metamaterials.

(a)
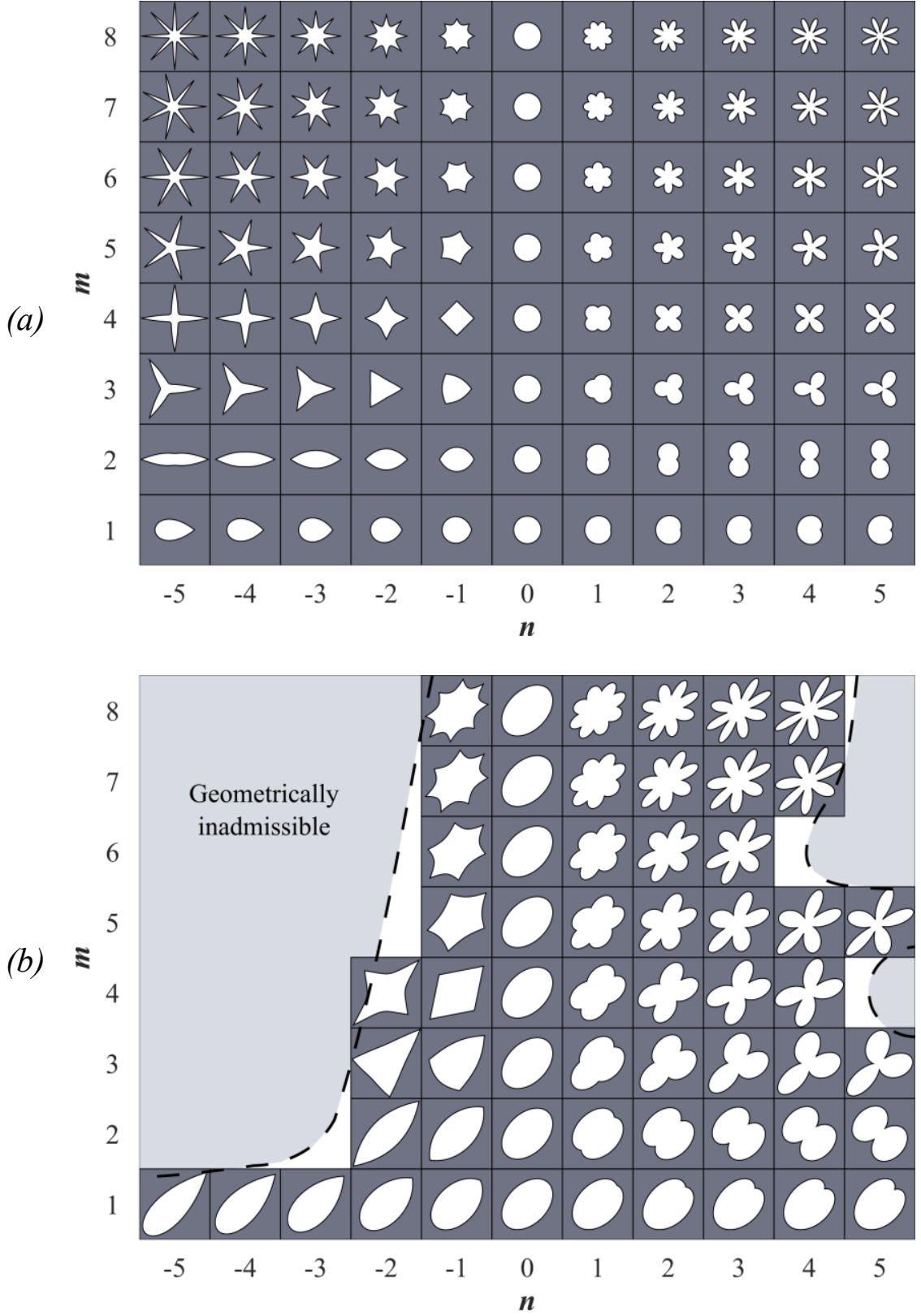

Figure 2.1: Sample RVEs of 2D architected cellular metamaterials for different topological parameters: (a) $\rho_{r}=0.88, S=1$ and $\beta=0^{\circ}$ and (b) $\rho_{r}=0.62, S=1.5$ and $\beta=45^{\circ}$. 


\subsubsection{D THIN-WALLED OPEN LATTICES}

Using closed 3D surface equations (such as 3D supershape formula) to generate 3D void shapes enables a parametric control over the pore geometry to create a variety of 3D RVEs for cellular materials. However, this method is generally unable of producing cells with low relative densities. To address this limitation, novel lightweight thin-walled open lattice architectures, based on 2D supershape pore topologies, are introduced and their effective thermal conductivities are compared with the thermal conductivity of $2 \mathrm{D}$ and $3 \mathrm{D}$ cellular materials. The $2 \mathrm{D}$ supershape pores are cut from each face of a hollow cubic RVE with a total relative wall thickness equal to $2 \sim 10 \%$ of the RVE's length ( $t_{r}=0.02 \sim 0.1$ ). To fulfill geometrical periodicity, opposite faces of RVE are cut by identical pore shapes. As illustrated in Fig. 2.2b, with three different 2D pore shapes on the faces of cubic RVE, the number of achievable 3D thin-walled open cells is much higher than their 2D closed-cell counterparts. Considering the fact that 3D computer-aided design (CAD) and finite element modelling are more computationally expensive than 2D RVEs, a tighter limit for the geometrical parameters of supershape pores is adopted $(1 \leq m \leq 4,-3 \leq n \leq 3$ and $S=1)$ and the pore's topological parameters are kept constant on all faces of the $3 \mathrm{D}$ thin-walled open lattices (Fig. 2.2c).

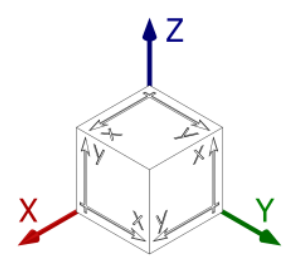

(a)

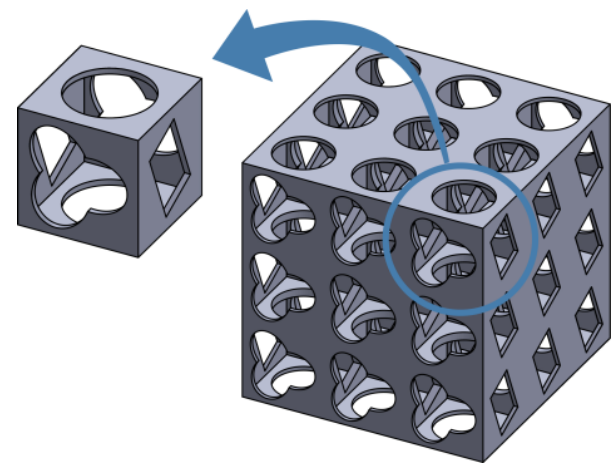

(b)

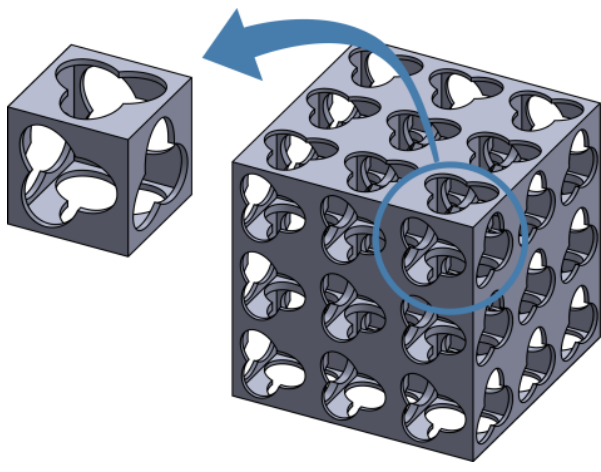

(c)

Figure 2.2: (a) Local coordinate system used for the creation of 2D supershape pores on each face of 3D RVE, (b) Possible thin-walled open lattice modeled in SolidWorks, and (c) Schematic view of a thin-walled open lattice considered in this study.

\subsubsection{D SHELLULAR RVES}

A Triply Periodic Minimal Surface (TPMS) is a non-self-intersecting surface in $\mathrm{R}^{3}$, which has a crystalline structure repeated in three independent directions, having a zero mean curvature at 
each point [46]. The presence of TPMS in natural materials, like biological membranes [47] and crystals [46], has inspired researchers to consider TPMS architectures in tissue engineering and biomimetic material design [48-51]. The Shellular term has been recently used in the literature to represent thin TPMS cellular shells. Among many known TPMSs, Schwarz's Primitive (P), Diamond (D), and Schoen's Gyroid (G) are selected here for analysis. These surfaces can be trigonometrically approximated using the following level surface equations [52]:

$$
\begin{array}{lll}
\boldsymbol{P}: & \cos x+\cos y+\cos z=f & (f=0,0.4,0.8) \\
\text { D: } & \sin x \sin y \sin z+\sin x \cos y \cos z+\cos x \sin y \cos z+ & (f=0) \\
& \cos x \cos y \sin z=f & (f=0) \\
\text { G: } \quad \cos x \sin y+\cos y \sin z+\cos z \sin x=f &
\end{array}
$$

We developed a MATLAB code to solve the level surface equations and determine the coordinates of several points on the surface. These points together with multiple cross-section curves, satisfying the surface equations, are firstly created inside SolidWorks using a Visual Basic code and then used to create a smooth surface. This part of the surface is then patterned to create the mid-surface of the Shellular RVE and the pattern is subsequently thickened to add the desired thickness (as shown in Fig. 2.A.2 in Appendix 2.A2). To focus on lightweight structures, five relative thicknesses $\left(t_{r}\right)$ from 2 to 10\% of RVE's length are considered for analysis. Figure 2.3 presents some selected Shellular architectures.

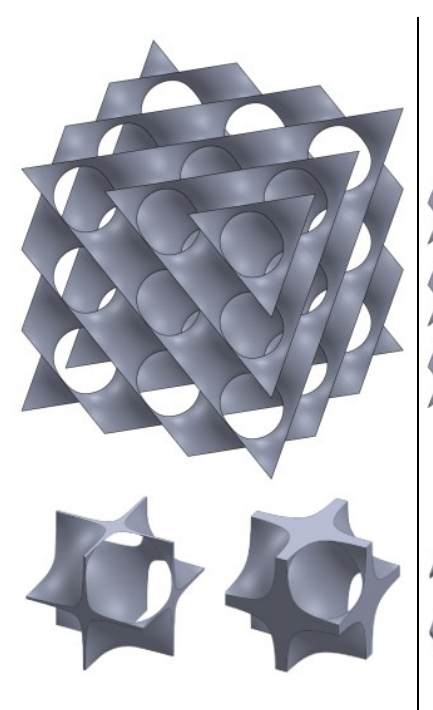

(a)

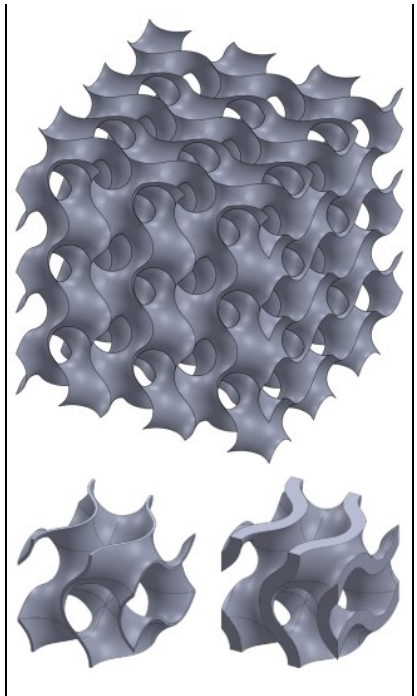

(b)

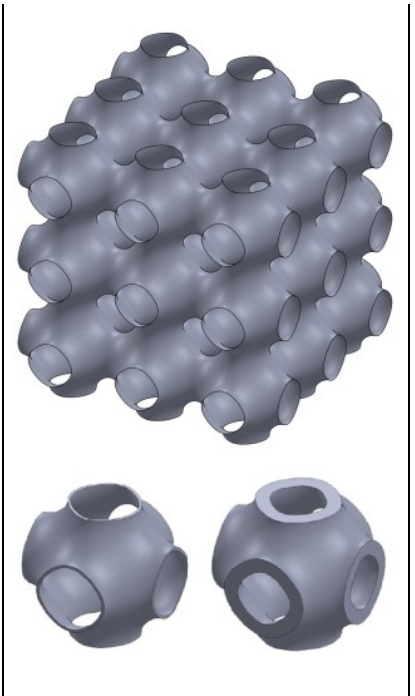

(c)

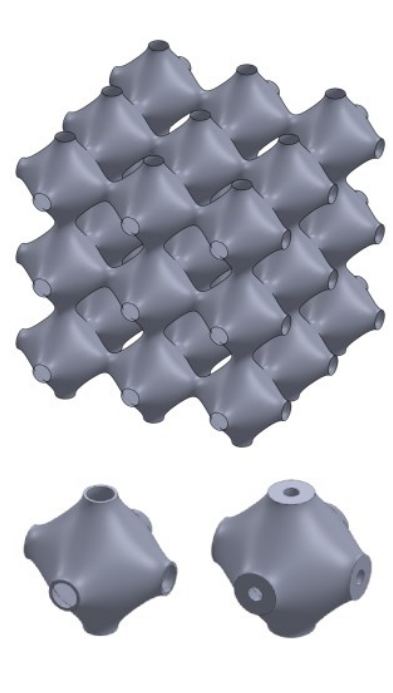

(d) 
Figure 2.3: $3 \times 3 \times 3$ TPMS cells together with two Shellular RVEs at 0.02 and 0.10 relative thicknesses: (a) Schwarz D, (b) Gyroid, (c) Schwarz P with $f=0$, and (d) Schwarz P with $f=$

0.8 .

\subsection{Predictive Methods}

Fourier's law assumes the following linear relation between the heat flux $(\overrightarrow{\mathrm{q}})$ and temperature gradient $(\vec{\nabla} \mathrm{T})$ through a symmetric thermal conductivity tensor $\left(\mathrm{K}^{\mathrm{eff}}\right)$ :

$$
\vec{q}=-K^{e f f} \vec{\nabla} T
$$

For a thermally isotropic homogenous solid material, $K^{e f f}$ tensor reduces to $K I$, where $I$ is the identity tensor and $K$ is the isotropic thermal conductivity. However, in the case of cellular materials, the overall thermal conductivity is generally anisotropic and depends on microarchitectural parameters of the cells [5].

Since the simple volumetric averaging approach is indifferent to cell architecture, it is unable to capture the anisotropic thermal properties of cellular materials leading to effective thermal conductivity overestimation. To address these shortcomings, several analytical and computational methods have been developed, among which the thermal-circuit method (or Resistor approach) has been widely used to predict the theoretical upper and lower bounds of thermal conductivity for a given cell topology [53]. More advanced numerical methods, e.g., computational homogenization, are required to exactly model the microarchitecture of cellular materials.

It should be noted that the contribution of heat transfer mechanisms other than conduction, i.e. radiation and convection, is assumed to be small and consequently neglected in this paper. For a cellular material made by a highly conductive matrix and empty voids or gaseous inclusions, this assumption is valid for small pore sizes working close to the ambient temperature [54-57].

\subsubsection{Analytical Modelling}

Using the analogy between thermal and electric fields, the thermal circuit method with parallel and series configurations is used to derive closed-form expressions for the upper and lower bounds of the thermal conductivity of cellular materials with rectangular or elliptic inclusion/pore. In this approach, the thermal gradient is analogous to electric voltage, the heat flow represents the electric 
current and the thermal resistance, being equal to the reciprocal of thermal conductivity for a unit cell, corresponds to electric resistance [44]. To establish this analytical model, Fig. 2.4 shows that the unit cell is divided into rectangular elements acting as thermal resistors while the heat flux is considered to flow along the $y$ direction and perfect thermal contact is assumed between the matrix (solid cell walls) and the filler (air for cellular materials).

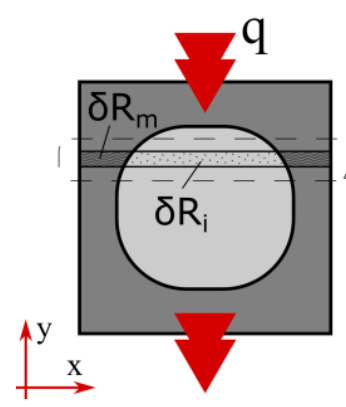

(a)

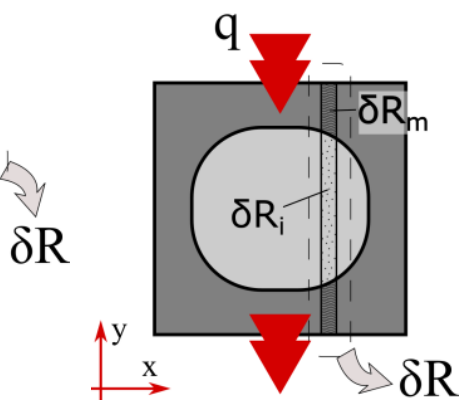

(b)

Figure 2.4: Schematic view of the thermal resistant elements for: (a) Horizontal iso-thermal

$$
\begin{gathered}
\text { lines where } \delta R^{y}=\left(\frac{1}{\delta R_{m}^{y}}+\frac{1}{\delta R_{i}^{y}}\right)^{-1} \text { and }, R^{y}=\sum \delta R^{y} \text { and (b) Vertical adiabatic lines with } \\
\delta R^{y}=\delta R_{m}^{y}+\delta R_{i}^{y} \text { and } R^{y}=\left(\sum \frac{1}{\delta R^{y}}\right)^{-1} .
\end{gathered}
$$

Closed-form thermal conductivity formulations are derived and presented in Table 2.2, where $k_{m}$ and $k_{i}$ are the thermal conductivity of the matrix and inclusion, respectively. In addition, $l$ is the dimension of the square RVE, $a$ and $b$ are the pore length and width along the $x$ and $y$ directions, respectively; $\lambda_{a}=a / l, \lambda_{b}=b / l, \lambda_{k}=K_{i} / K_{m}(<1), \theta=\operatorname{atan}\left(\sqrt{\left(\left(1-\lambda_{k}\right) \lambda_{a}\right)^{-2}-1}\right.$ and $A=$ $\lambda_{b}\left(1 / \lambda_{k}-1\right)$. It can be deduced that the 'Vertical adiabatic lines' expressions for both rectangular and elliptic geometries reduce to $K_{y y}^{e f f} / K_{m}=1-\lambda_{a}$ when $K_{i}=0$, which corresponds to cellular materials with empty (vacuum) pores. The relative density of the matrix can be calculated as $\left(\rho_{m}\right)_{r}=\left(l^{2}-a b\right) / l^{2}=1-\lambda_{a} \lambda_{b}$ and $\left(\rho_{m}\right)_{r}=\left(l^{2}-\pi a b / 4\right) / l^{2}=1-\pi \lambda_{a} \lambda_{b} / 4$, for cells with rectangular and elliptic inclusion/pore, respectively. As symmetry in the selected architectures of matrix and inclusion/pore dictates, the off-diagonal terms of the thermal conductivity tensor are zero $\left(K_{x y}^{e f f}=0\right)$ and the thermal conductivity in the $x$ and $y$ directions are equal. 
Table 2.2: Closed-form expressions for $K_{y y}^{e f f} / K_{m}$ of cells made by thermally isotropic and homogeneous matrix and filler material (inclusion), having rectangular and elliptic inclusion/pore shapes, under the assumptions of 'Horizontal iso-thermal lines' or 'Vertical adiabatic lines'.

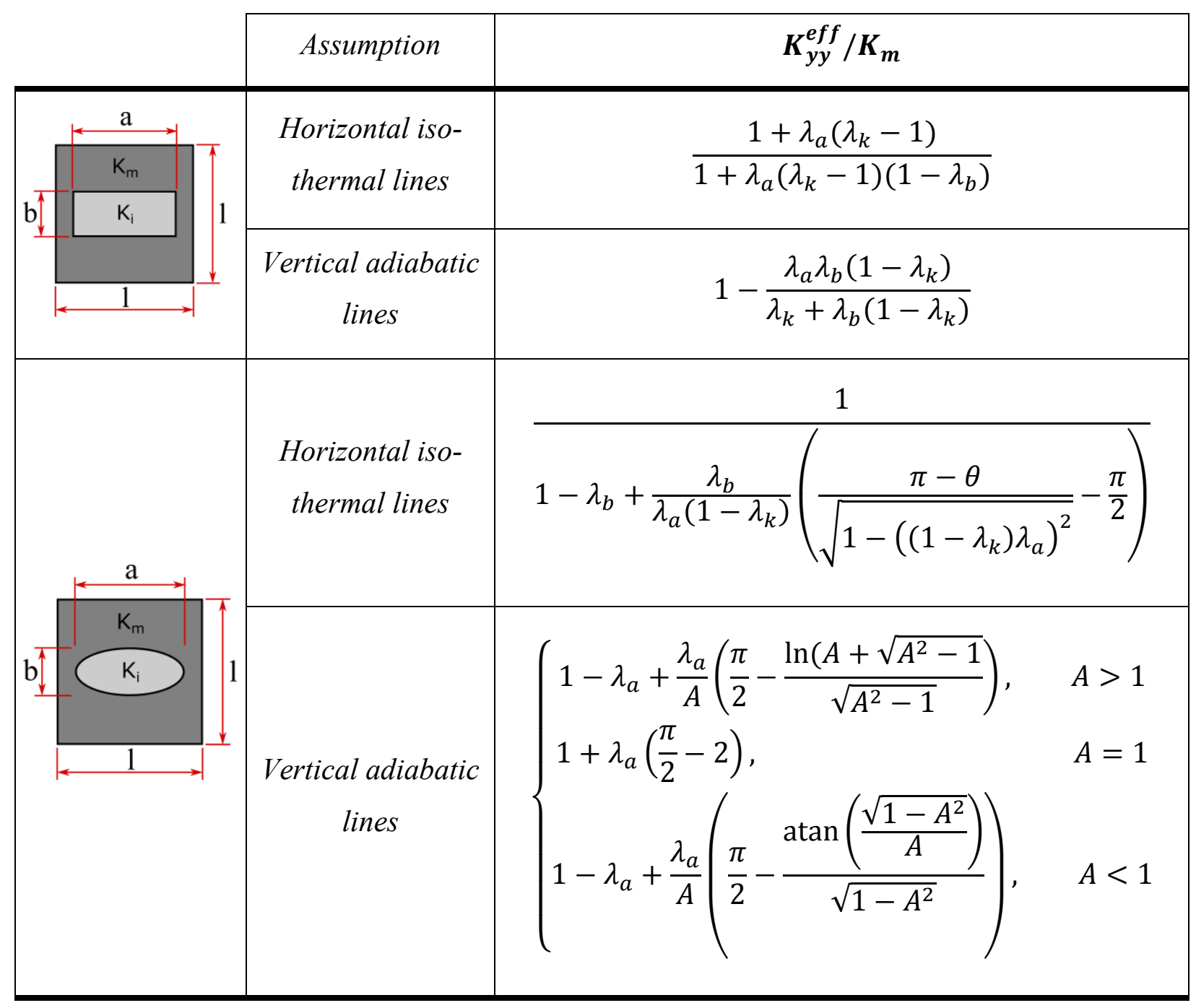

\subsubsection{Computational Standard Mechanics Homogenization}

Under the assumption that the RVE of a periodic cellular material is repeated in all three directions and the RVE is far from the boundaries, the following periodic boundary conditions (Eq. (2.4)), together with independent unit thermal gradients (Eq. (2.5)), are adopted on the boundaries [16]:

Periodicity in $x$ direction: 


$$
T\left(x_{0}, y, z\right)-T\left(x_{0}+l_{x}, y, z\right)=l_{x}\left(\frac{\partial T}{\partial x}\right)_{i}
$$

Periodicity in $y$ direction:

$$
T\left(x, y_{0}, z\right)-T\left(x, y_{0}+l_{y}, z\right)=l_{y}\left(\frac{\partial T}{\partial y}\right)_{i}
$$

Periodicity in $z$ direction:

$$
T\left(x, y, z_{0}\right)-T\left(x, y, z_{0}+l_{z}\right)=l_{z}\left(\frac{\partial T}{\partial z}\right)_{i}
$$

Independent unit thermal gradients:

$$
(\overline{\nabla T})_{i}=\left(\overline{\frac{\partial T}{\partial x}}, \overline{\partial T} \frac{\overline{\partial T}}{\partial y}, \frac{\overline{\partial z}}{\partial z}\right)_{i}= \begin{cases}(1,0,0), & i=1 \\ (0,1,0), & i=2 \\ (0,0,1), & i=3\end{cases}
$$

where $i$ is the thermal loading case number and $\mathrm{x}_{0}, \mathrm{y}_{0}$ and $\mathrm{z}_{0}$ are the locations of the three negative faces of the cubic RVE, while $\mathrm{l}_{\mathrm{x}}, \mathrm{l}_{\mathrm{y}}$ and $\mathrm{l}_{\mathrm{z}}$ represent RVE's dimensions along the $x, y$ and $z$ axes, and $\overline{\nabla T}$ is the average thermal gradient applied to the RVE's boundaries to calculate the microscopic thermal gradients inside the unit cell. Since the RVE is a unit square in 2D analysis and a unit cube in 3D analysis, Eqs. (2.4) and (2.5) can be further simplified to:

$$
\left(\begin{array}{l}
T\left(x_{0}, y, z\right)-T\left(x_{0}+1, y, z\right) \\
T\left(x, y_{0}, z\right)-T\left(x, y_{0}+1, z\right) \\
T\left(x, y, z_{0}\right)-T\left(x, y, z_{0}+1\right)
\end{array}\right)_{i}=\left(\begin{array}{l}
1 \\
0 \\
0
\end{array}\right)_{i=1},\left(\begin{array}{l}
0 \\
1 \\
0
\end{array}\right)_{i=2},\left(\begin{array}{l}
0 \\
0 \\
1
\end{array}\right)_{i=3}
$$

For 2D analysis, all terms in the $z$ dimension are discarded, leading to two independent thermal loading cases. The energy balance equation, i.e. energy equation combined with Fourier heat conduction, is solved over the RVE using the finite element method (FEM). The effective thermal conductivity of the cellular materials can be calculated by the volumetric averaging of the resultant heat flux based on the standard mechanics homogenization as [58]:

$$
\bar{K}_{i j}=\frac{1}{V_{R V E}} \int K_{i k} M_{k j}^{T} d V_{R V E} \quad(i, j, k=1,2,3)
$$

where $\bar{K}_{\mathrm{ij}}$ is the effective thermal conductivity tensor, $\mathrm{V}_{\mathrm{RVE}}$ represents the RVE volume, $\mathrm{K}_{\mathrm{ik}}$ is the local thermal conductivity tensor, and the $\mathrm{M}^{\mathrm{T}}$ tensor relates the average and local temperature gradients by $\nabla T=M^{T} \overline{\nabla T}$. 


\subsection{RESULTS AND DISCUSSION}

Starting with verification of the numerical homogenization scheme, the obtained effective thermal conductivities of periodic cubic RVEs with spherical pores in the well-known simple cubic (SC), face-centered cubic (FCC) and body-centered cubic (BCC) arrangements are compared with the findings in literature. Figure 2.A.3 (in Appendix 2.A3) demonstrates the good agreement between our numerical results with those reported in [20].

To show the effect of filler (inclusion) material on the overall thermal conductivity and to examine the validity of neglecting the thermal conductivity of the gas inside the pore of cellular materials, the homogenized thermal conductivity of a square cell with an empty square pore is benchmarked against the effective thermal conductivity of the same cell filled with air at room temperature. As shown in Fig. 2.5, for relative densities higher than $10 \%$, the air's thermal conductivity can be neglected without affecting the effective thermal conductivity when the thermal conductivity of the matrix is several orders of magnitude higher than the air, such as in metallic cellular materials.

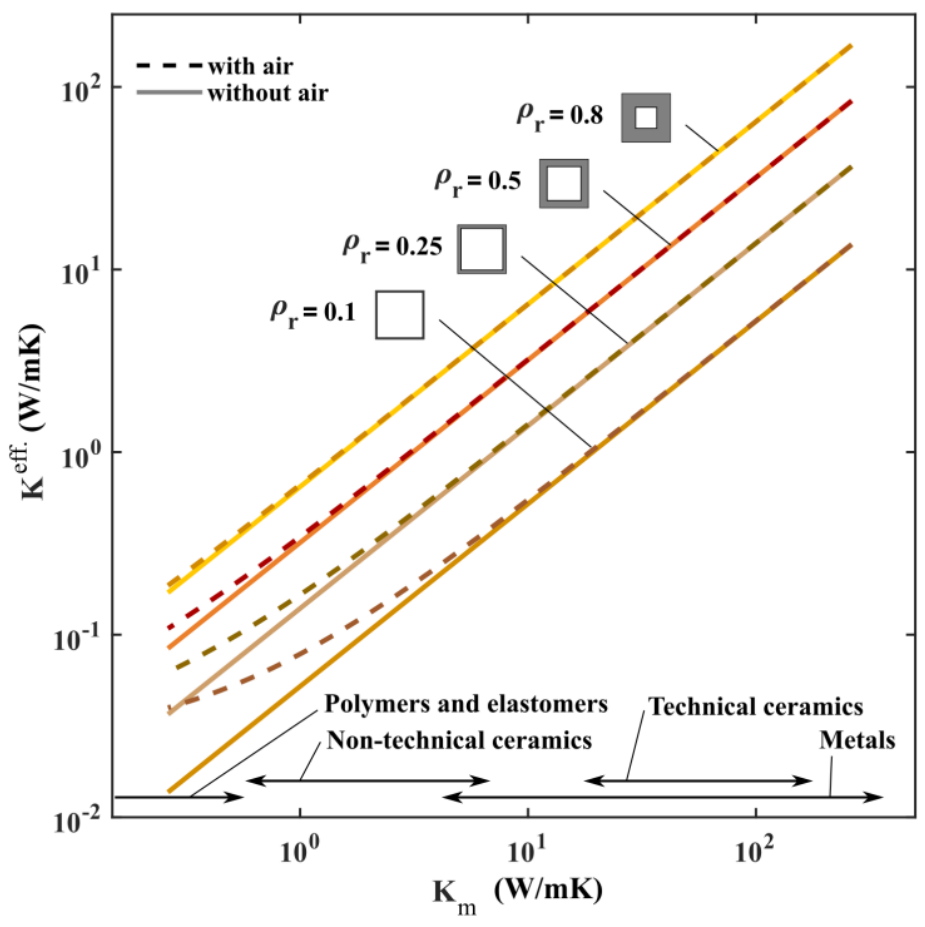

Figure 2.5: Effective thermal conductivity of air-filled $\left(k_{\text {air }}=0.0263 \mathrm{~W} / \mathrm{mK}\right)$ and empty $\left(k_{i}=0\right)$ cellular materials for a square pore shape versus the thermal conductivity of the solid matrix (log-log scale). 
Under the assumption of neglecting the air, the closed-form expressions presented in Table 2.2 for the cellular materials with rectangular and elliptic pore topologies are compared with the results obtained by standard mechanics homogenization. As shown in Fig. 2.6, for all pore's aspect ratios $\left(\frac{\lambda_{a}}{\lambda_{b}}=1,2\right.$ and 3$)$, the distances between the higher and lower bounds of the effective thermal conductivity of the cells with elliptic pores are larger than those for rectangular pores. Moreover, by increasing the relative density, the distance between the theoretical bounds increases. In this case, although the pore topology is accounted to derive the theoretical upper and lower bounds, the simplifying assumptions made in deriving the closed-form formula based on the thermal-circuit method make it impossible to accurately predict the effective thermal conductivity. Close to the smallest possible relative density for each pore aspect ratio, the effective thermal conductivity of cells with elliptic pores shows sharper drops compared to rectangular pores. This is partially because of the higher decreasing rate of the minimum wall thickness with decreasing relative density for the cells with elliptic pores.

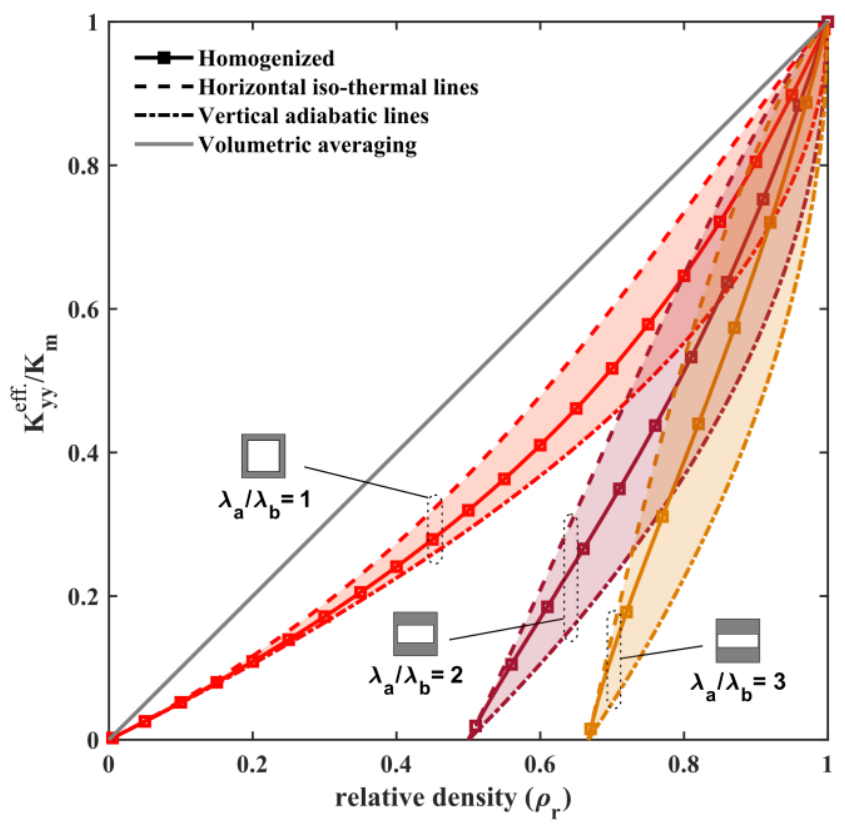

(a)

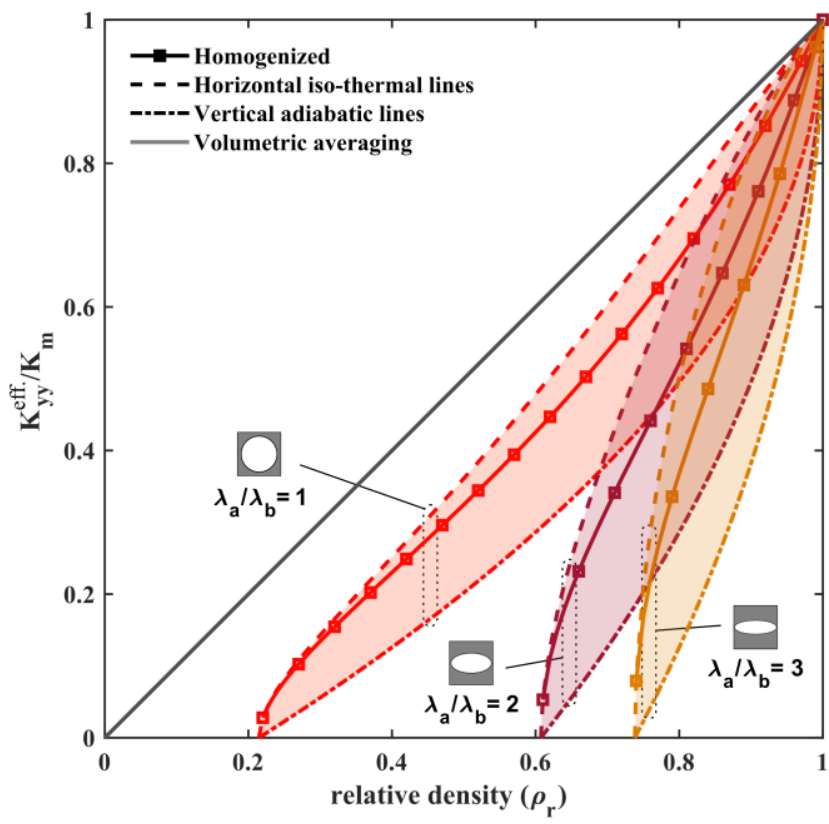

(b)

Figure 2.6: Theoretical upper and lower bounds along with computational homogenized values of the thermal conductivity along $y$ direction, normalized by the thermal conductivity of the solid matrix: (a) rectangular, and (b) elliptic pores. 
The standard mechanics homogenization is applied to predict the thermal conductivity of 2D architected cellular metamaterials with supershape pore topologies. We consider the variation of the pore's scaling $S$ between 1 and 3.5 (with 0.5 increment) and the pore's rotations in the range of $0^{\circ}$ to $90^{\circ}$ (with $7.5^{\circ}$ increment) to better show the effect of the pore shape and pore rotation on tuning the thermal properties of engineered porous materials. The in-plane effective thermal conductivity generally shows anisotropic properties in both $x$ and $y$ directions. As a result, the effective principal thermal conductivities ( $K_{1}$ as the maximum and $K_{2}$ as the minimum) are presented in Fig. 2.7 instead of $K_{x x}, K_{y y}$ and $K_{x y}$. Some representative relative densities are highlighted with different colors to show the achievable range of $K_{1}$ and $K_{2}$ by selected pore topologies. The large area of $K_{1}$ versus $K_{2}$ for each relative density allows the engineering of architected cellular materials while keeping the weight constant.

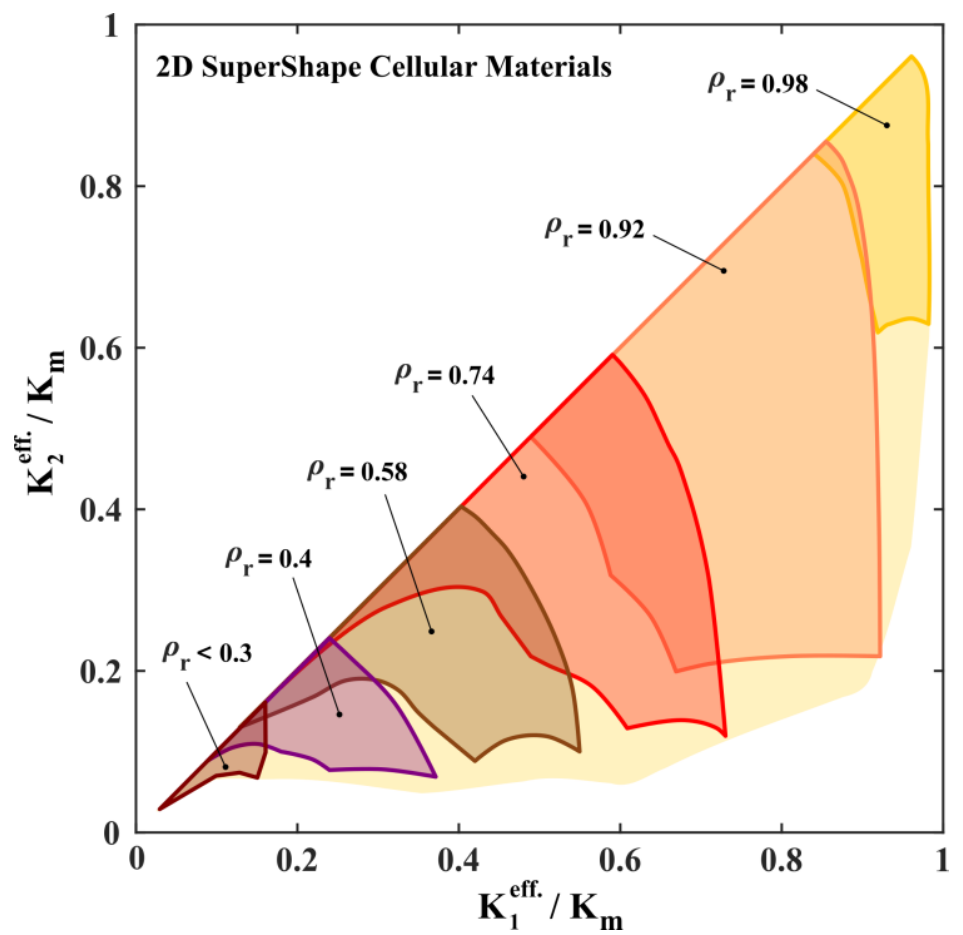

Figure 2.7: Normalized effective principal thermal conductivities predicted by standard mechanics homogenization for 2D supershape RVEs.

As mentioned earlier, we consider RVEs with enclosed 2D supershape pores which have wall thicknesses larger than 0.02 times the RVE's length. For this RVE, reducing the relative density pushes the boundaries of the pore towards the cell edges, which makes scaling and rotation impossible at small relative densities. This results in square-like pore topologies with isotropic in- 
plane effective thermal conductivities for relative densities smaller than 0.2. This behavior can be observed on the far left side of Fig. 2.7, in which $K_{1}^{e f f}$ and $K_{2}^{e f f}$ are getting closer with decreasing relative density, leading towards thermal isotropy. As an example, Fig. 2.8 exhibits two RVEs with relative densities below 0.2 .

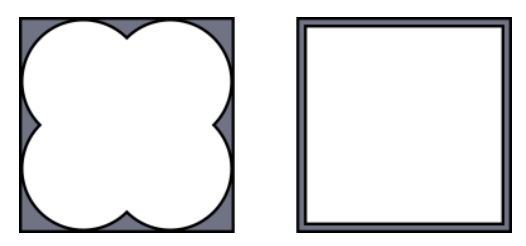

Figure 2.8: Two RVEs with relative densities below 0.2.

While decreasing the relative density usually leads to isotropic thermal conductivity for square RVEs with a supershape pore, other cell architectures might enforce anisotropic thermal conductivity even at low relative densities. Figure 2.9 shows an example for an RVE which has a pore size several times larger than the RVE's dimensions. While for thermally isotropic materials the ratio of the maximum to minimum principal thermal conductivities is equal to one, the architected cell presented in Fig. 2.9 exhibits a highly anisotropic effective thermal conductivity, with an in-plane thermal conductivity ratio of $K_{1}^{e f f} / K_{2}^{e f f}$ close to 60 .
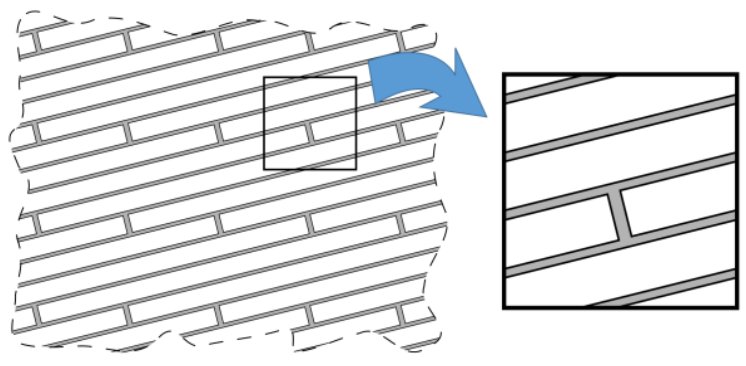

Figure 2.9: An example of an RVE with $\rho_{r}=0.132$ and a highly anisotropic effective thermal conductivity ( $K_{1}^{e f f} / K_{m}=0.122$ and $K_{2}^{e f f} / K_{m}=0.002$ ). Rectangular pore's specifications are: 19.1 aspect ratio and $14^{\circ}$ rotation angle.

The results presented in Fig. 2.10 show how the effective thermal conductivity of $2 \mathrm{D}$ supershape cellular metamaterials can be tuned by changing the shape parameters ( $m, n, S, \beta$ and $\rho_{r}$ ) of supershape pore. A detailed analysis of the results obtained by standard mechanics homogenization, presented in Fig. 2.10, shows that: 
- Narrower relative density range is possible generally with $n<0$, which is the main reason for the smaller region covered in the $K_{2}^{e f f}$ vs. $K_{1}^{e f f}$ plot for $n<0$ compared to $n>0$.

- $\quad K_{x y}^{e f f}$ for all supershape pore topologies with $\beta=0^{\circ}$ is zero, which can be justified by the fact that the defined 2D RVEs (supershape pore within square cells) are symmetric about the $x$ axis, which in turn makes $K_{x y}^{e f f}$ zero, identifying $K_{x x}^{e f f}$ and $K_{y y}^{e f f}$ as the principal thermal conductivities. Similarly for $\beta=45^{\circ}$, the RVEs are symmetric about one of the diagonals of the square cell, thus off-diagonal terms of the thermal conductivity tensor must be zero along this direction.

- With the same relative density, rotating the elongated pore in the $x$ direction $(S>1)$ by $45^{\circ}$ decreases the $K_{1}^{\text {eff }} / K_{2}^{\text {eff }}$ ratio. It should be noted that the pore dimensions remain unchanged by keeping the relative density constant. Therefore, rotating the pores by $45^{\circ}$ aligns them in the diagonal direction increasing the distance between the pores of the adjacent cells and providing more space for heat to flow.

- $\quad K_{1}^{e f f}$ and $K_{2}^{e f f}$ of cells with lower pore elongation $(S)$ and higher relative densities are less sensitive to pore rotation than cells with larger $S$ values or cells with smaller relative densities. For instance, for $S=1.5$ and relative densities above 0.95 , pore rotation does not change the principal effective thermal conductivities. However, this is not true for lower relative densities or larger $S$ values.

- Regardless of rotation angle, for $S=1$, all cases with $m=4$ or 8 or RVEs with $n=0$ (circular pore) are thermally isotropic. This is a result of the four-fold rotational symmetry of these supershape RVEs which leads to $K_{x x}^{e f f}=K_{y y}^{e f f}$ and $K_{x y}^{e f f}=0$.

- For $m=2$ and $n>0$, the supershape pore is initially elongated in the $y$ direction. For other cases, increasing $S$ generally leads to architected metamaterials with highly anisotropic effective thermal conductivity, at the cost of not being able to achieve lower relative densities. 


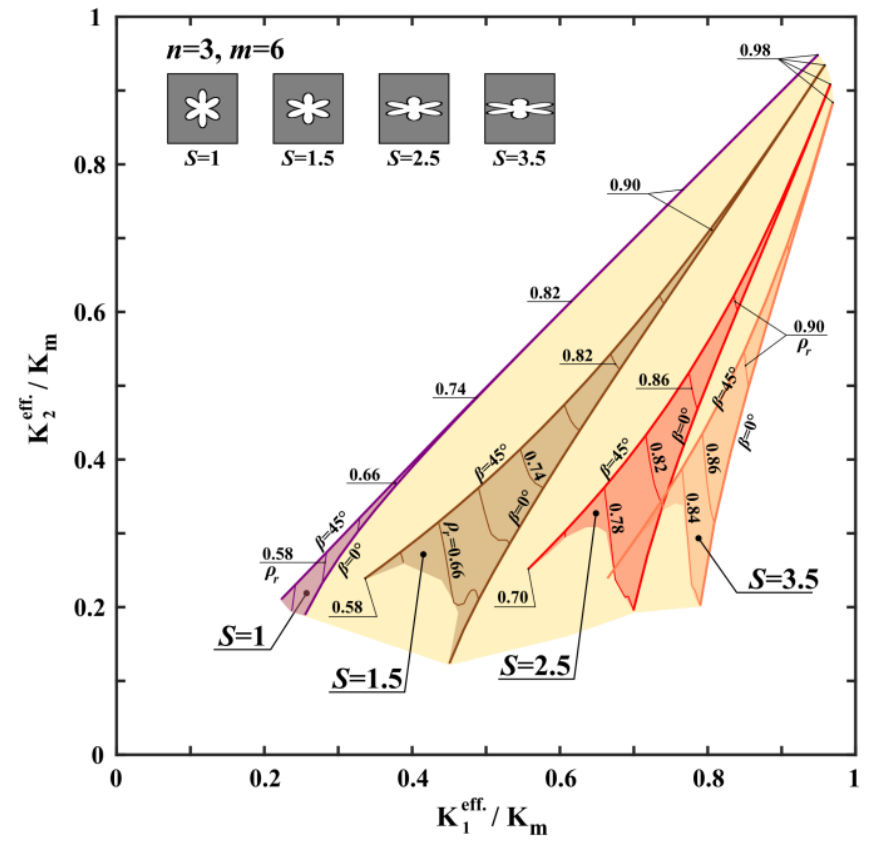

(a)

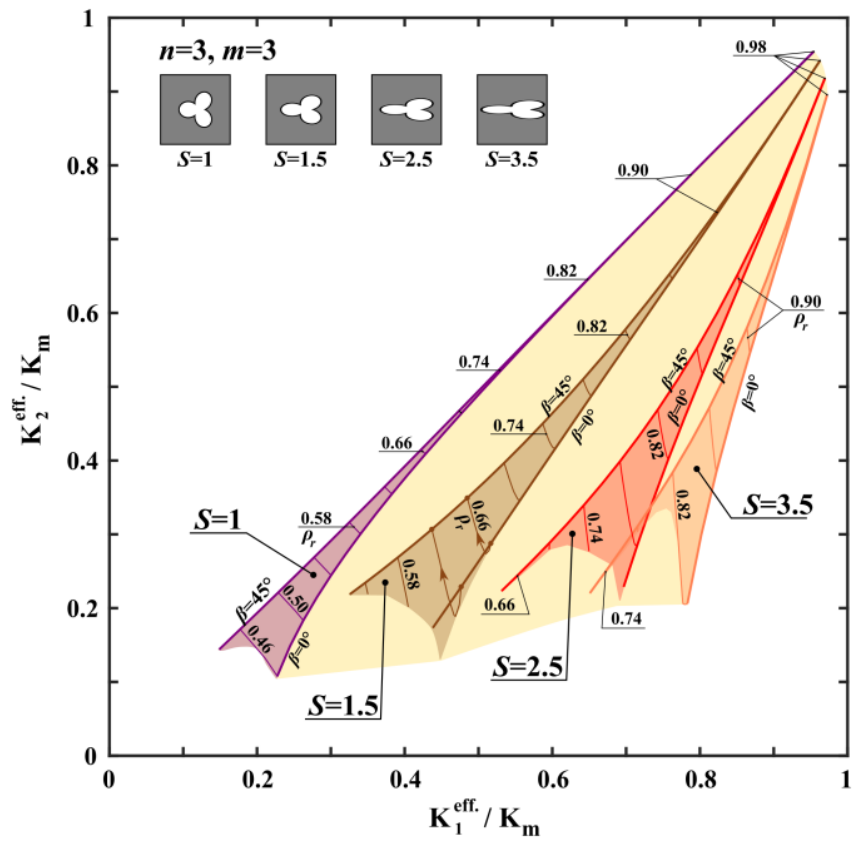

(b)

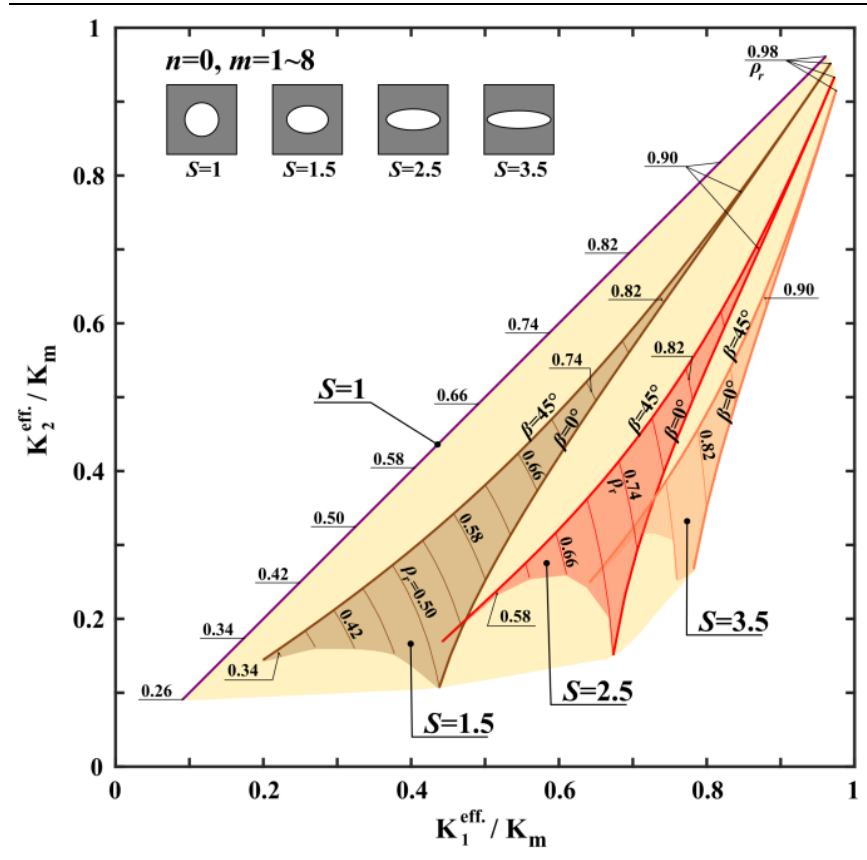

(c)

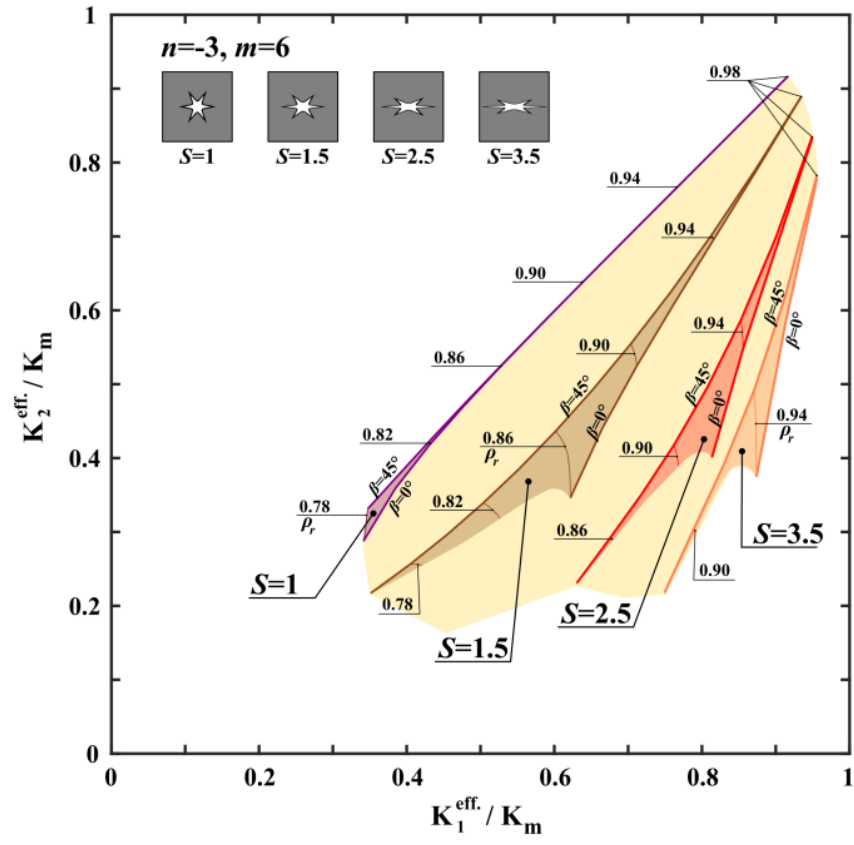

(d) 


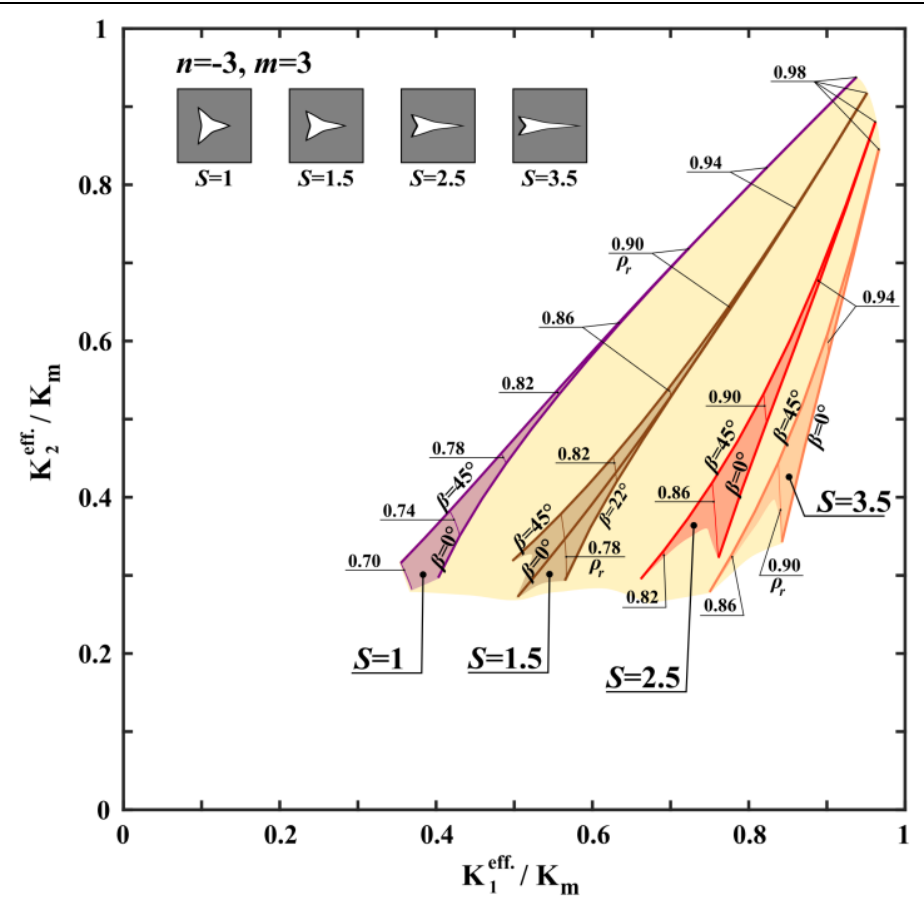

(e)

Figure 2.10: Effective principal thermal conductivities of $2 D$ supershape RVEs for different sets of topological parameters: a) $[n=3, m=6]$, b) $[n=3, m=3]$, c) $n=0, d)[n=-3, m=6]$, and e) $[n=-$ $3, m=3]$.

An interesting, but not-widely sought, thermal property of advanced architected materials is off-diagonal terms of the thermal conductivity matrix; i.e. $K_{x y}, K_{x z}$ and $K_{y z}$. While these offdiagonal terms are zero for thermally isotropic materials and applying temperature gradients in any direction only results in heat flow in that direction, applying temperature gradient along one direction on thermally anisotropic metamaterials can also result in heat flow in other directions. For instance, temperature gradient along the $x$ direction in thermally anisotropic 2D materials with non-zero $K_{x y}$ can also create heat flow in either positive or negative $y$ direction. Figure 2.11 shows $K_{x y}^{e f f}$ for all analyzed 2D supershape RVEs with respect to their relative density. Inspecting cases with higher $K_{x y}^{e f f}$ shows that highly elongated pores aligned along one of the RVE's diagonals lead to a significant increase of the magnitude of the off-diagonal thermal conductivity term. 


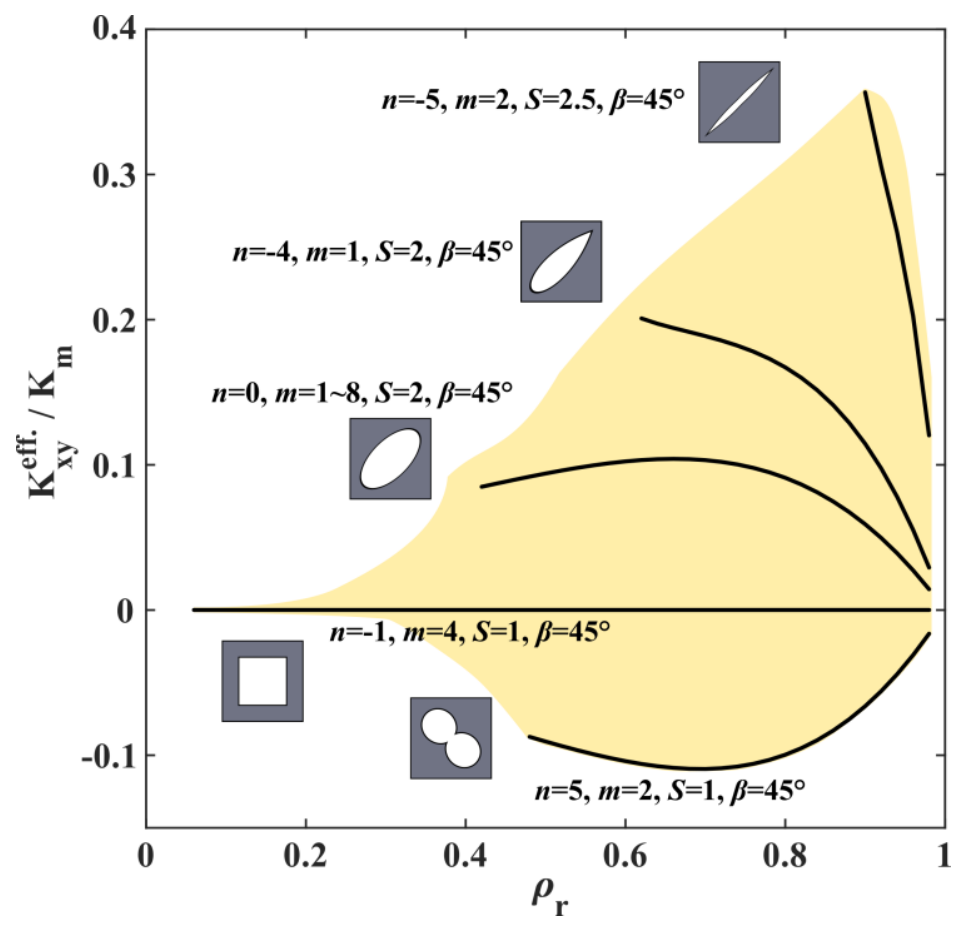

Figure 2.11: Effective off-diagonal term in the thermal conductivity tensor for $2 D$ supershape RVES with respect to the relative density obtained by standard mechanics homogenization.

It should be noted that by rotating a thermally anisotropic material with respect to a coordinate system, components of its thermal conductivity tensor change; hence, the materials' orientation (not to be confused with pore's rotation) can also be considered as an approach to control the thermal conductivity of an anisotropic material. To obtain the thermal conductivity tensor of the rotated material, coordinate transformation can be used [28, 41]. The Mohr's circle (Fig. 2.12) can also be used as an alternative.

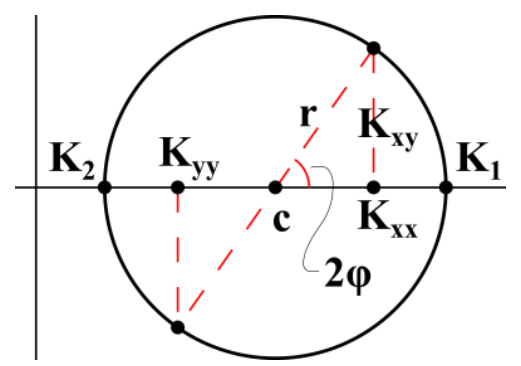

Figure 2.12: Using the Mohr's circle to calculate the thermal conductivity components for a rotated anisotropic material.

It is worth mentioning that with the considered minimum wall thicknesses of $2 \mathrm{D}$ cellular metamaterials, all 2D supershape RVEs in this research have non-zero thermal conductivities in 
all directions. As a result, the distance between the Mohr's circle center and the origin (c) in Fig. 2.12 is always larger than the Mohr's circle radius $(r)$ meaning that $K_{2}$ must be positive. Considering that $\max \left(K_{x y}\right)=r$, and $\max \left(K_{x x}, K_{y y}\right) \geq c$, the maximum of the $K_{x x}$ and $K_{y y}$ must always be higher than $K_{x y}$, while $\min \left(K_{x x}, K_{y y}\right)$ can be smaller, larger or equal to $K_{x y}$ depending on the rotation angle of the material and the magnitudes of $c$ and $r$. Interestingly, for $K_{x y}>K_{x x}$, applying a temperature gradient in the $y$ direction would make higher heat transfer in the $x$ direction, compared to applying the same temperature gradient in the $x$ direction. It can be easily shown that when $c /(r \sqrt{2})>1$, which by replacing $c$ by $\left(K_{1}+K_{2}\right) / 2$ and $r$ by $\left(K_{1}-K_{2}\right) / 2$, simplifies to $K_{1} / K_{2}<(\sqrt{2}+1) /(\sqrt{2}-1)$, for all rotation angles of the material, the minimum of $K_{x x}$ and $K_{y y}$ is always larger than $K_{x y}$. However, when $c /(r \sqrt{2}) \leq 1$, or equivalently $K_{1} / K_{2} \geq$ $(\sqrt{2}+1) /(\sqrt{2}-1)$, which is the case for large $K_{1}$ and small $K_{2}$ values, a rotation angle can be found at which $\min \left(K_{x x}, K_{y y}\right)<K_{x y}$. Inspecting this criteria among all the effective thermal conductivities of the 2D supershape RVEs, we found few cases, e.g., $[n=2, m=4, S=2, \beta=0$, $\left.\rho_{r}=0.6\right]$ and $\left[n=2, m=4, S=2.5, \beta=0, \rho_{r}=0.68\right]$, which have $K_{x y}^{e f f}$ marginally greater than their $K_{y y}^{e f f}$ when these architected materials are rotated by $-22.5^{\circ}$. High $K_{x y}$ values open up the possibility of controlling the net heat flow in the $x$ direction by tuning the temperature gradient in the $y$ direction. Table 2.3 presents a case study where the net heat flow in the $x$ direction has been increased (Case b) or reversed (Case $c$ ) by applying different temperature gradients along the $y$ direction of a cellular medium made of a $-45^{\circ}$ rotated supershape pore with $[n=2, m=4, S=2.5$, $\left.\beta=0, \rho_{r}=0.68\right]$. The cellular part is a unit square with its corners cut by $0.1 \mathrm{~m}$, while the thermal conductivity of the thermally isotropic base material is assumed to be $100 \mathrm{~W} / \mathrm{mK}$, with the aforementioned architecture, the effective thermal conductivity of the cellular medium is determined as: $K_{x x}^{e f f}=K_{y y}^{e f f}=37.2 \mathrm{~W} / \mathrm{mK}$ and $K_{x y}^{e f f}=-26.8 \mathrm{~W} / \mathrm{mK}$. For all cases, the left wall's temperature $\left(\mathrm{T}_{1}\right)$ is set at $6^{\circ} \mathrm{C}$, while the temperature of the right wall $\left(\mathrm{T}_{\mathrm{r}}\right)$ is $4^{\circ} \mathrm{C}$. In Case $a$, all the walls are insulated $(q=0)$ except the left and right walls. Positive temperature difference in the $y$ direction is applied in Case $b$ by setting the top wall's temperature $\left(\mathrm{T}_{\mathrm{t}}\right)$ at $10^{\circ} \mathrm{C}$ and the bottom wall's temperature $\left(\mathrm{T}_{\mathrm{b}}\right)$ at $0^{\circ} \mathrm{C}$. In Case $c$, the top and bottom wall temperatures of Case b are swapped so that the temperature difference applied in the $y$ direction is negative. Steady-state heat conduction simulation is conducted using ANSYS Fluent and temperature distribution, together 
with the effective heat flux passing through the left $\left(\mathrm{q}_{1}\right)$, right $\left(\mathrm{q}_{\mathrm{r}}\right)$, top $\left(\mathrm{q}_{\mathrm{t}}\right)$ and bottom $\left(\mathrm{q}_{\mathrm{b}}\right)$ walls of the part are presented in Table 2.3, in which as of convention, positive sign for heat flux stands for inward and negative sign indicates outward heat fluxes. The observed coupling between the heat fluxes in both $x$ and $y$ directions in Case $b$ and Case $c$, which is caused by the non-zero $K_{x y}^{e f f}$, highlights the importance of the off-diagonal components of the thermal conductivity tensor. Failing to consider this coupling might result in completely different temperature distribution and inaccurate conclusions. For instance, in Case $c$ although the imposed temperature difference in the $x$ direction is negative, the apparent heat flow in this direction is also negative. Referring to Eq. (2.3), this leads to deducing a negative thermal conductivity for the cellular part, if the aforementioned coupling is ignored.

Table 2.3: Thermal response of a square cellular part made by an anisotropic cellular architecture, subjected to a negative temperature gradient in the $x$ direction $\left(T_{l}>T_{r}\right)$ and different boundary conditions in the y direction. Thick solid black lines represent insulated walls.

\begin{tabular}{|c|c|c|c|}
\hline$\stackrel{\mathrm{y}}{\mathrm{x}}$ & Case a & Case b & 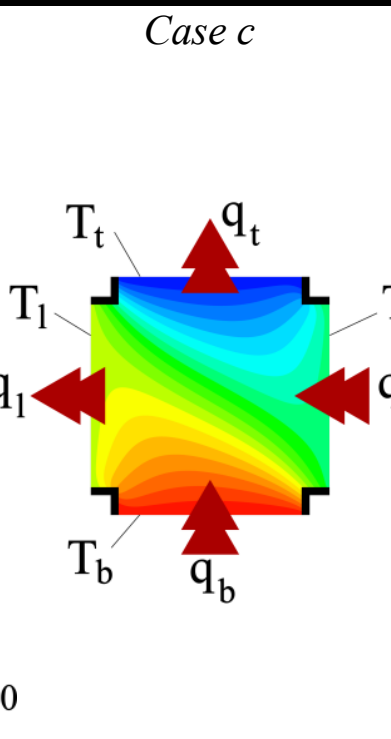 \\
\hline $\begin{array}{l}\text { Boundary } \\
\text { Conditions }\end{array}$ & $\begin{array}{l}\mathrm{T}_{1}=6^{\circ} \mathrm{C}, \mathrm{T}_{\mathrm{r}}=4^{\circ} \mathrm{C} \\
\mathrm{q}_{\mathrm{b}}=\mathrm{q}_{\mathrm{t}}=0 \mathrm{~W} / \mathrm{m}^{2}\end{array}$ & $\begin{aligned} \mathrm{T}_{1} & =6^{\circ} \mathrm{C}, \mathrm{T}_{\mathrm{r}}=4^{\circ} \mathrm{C} \\
\mathrm{T}_{\mathrm{b}} & =0^{\circ} \mathrm{C}, \mathrm{T}_{\mathrm{t}}=10^{\circ} \mathrm{C}\end{aligned}$ & $\begin{array}{c}\mathrm{T}_{1}=6^{\circ} \mathrm{C}, \mathrm{T}_{\mathrm{r}}=4^{\circ} \mathrm{C} \\
\mathrm{T}_{\mathrm{b}}=10^{\circ} \mathrm{C}, \mathrm{T}_{\mathrm{t}}=0^{\circ} \mathrm{C}\end{array}$ \\
\hline $\begin{array}{l}\text { heat fluxes } \\
{\left[\mathrm{W} / \mathrm{m}^{2}\right]}\end{array}$ & $q_{1}=43.2, q_{r}=-43.2$ & $\begin{array}{l}\mathrm{q}_{1}=280.2, \mathrm{q}_{\mathrm{r}}=-280.2 \\
\mathrm{q}_{\mathrm{b}}=-429.2, \mathrm{q}_{\mathrm{t}}=429.2\end{array}$ & $\begin{array}{c}\mathrm{q}_{1}=-123.9, \mathrm{q}_{\mathrm{r}}=123.9 \\
\mathrm{q}_{\mathrm{b}}=349, \mathrm{q}_{\mathrm{t}}=-349\end{array}$ \\
\hline
\end{tabular}


As shown in Fig. 2.13, by adding a small thickness to 2D supershape RVEs and assembling them in a predefined 3D arrangement, thin-walled open lattices are introduced. Using a finite element simulation in ANSYS APDL to conduct standard mechanics homogenization, effective thermal conductivity of these lightweight 3D architected RVEs is then determined.
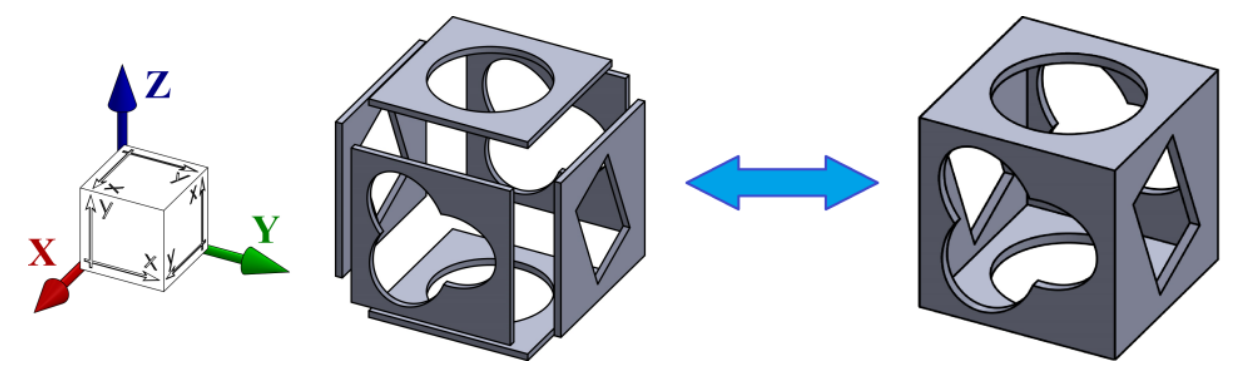

Figure 2.13: Assembling or disassembling a general thin-walled open lattice RVE.

To show the influence of pore topology of one side on the heat flow through the other sides of the thin-walled RVE, a simple mathematical model is derived based on superposition, in which heat flow through different RVE sides is assumed independent, and any heat flow or thermal resistance normal to all sides are neglected. Under these assumptions, all six RVE sides can be treated as separate entities as depicted in Fig. 2.13. Consequently, the effective thermal conductivity can be determined by summing up the contributions of these individual sides. Assuming that the effective in-plane thermal conductivities of the faces of the 3D RVE (which are 2D supershape RVEs) in their 2D local coordinate systems are given by $K_{z x f a c e}^{e f f} / K_{m}=$ $\left[\begin{array}{ll}a 3 & c 3 \\ c 3 & b 3\end{array}\right]_{\text {local }}, K_{y z \text { face }}^{\text {eff }} / K_{m}=\left[\begin{array}{ll}a 2 & c 2 \\ c 2 & b 2\end{array}\right]_{\text {local }}$, and $K_{x y f a c e}^{e f f} / K_{m}=\left[\begin{array}{ll}a 1 & c 1 \\ c 1 & b 1\end{array}\right]_{\text {local }}$, the overall thermal conductivity of the $3 \mathrm{D}$ thin-walled open RVE is calculated as:

$$
K_{3 D}^{e f f} / K_{m} \approx t_{r}\left[\begin{array}{ccc}
a 1+b 3 & c 1 & c 3 \\
c 1 & b 1+a 2 & c 2 \\
c 3 & c 2 & a 3+b 2
\end{array}\right]
$$

where the relative wall thickness $t_{r}$ is defined as the total wall thickness divided by RVE's length. Since the same pore topologies are applied to all sides of the thin-walled RVE, Eq. (2.8) can be further simplified as:

$$
K_{3 D}^{e f f} / K_{m} \approx t_{r}\left[\begin{array}{ccc}
a 1+b 1 & c 1 & c 1 \\
c 1 & b 1+a 1 & c 1 \\
c 1 & c 1 & a 1+b 1
\end{array}\right]
$$


This equation satisfies $K_{x x}=K_{y y}=K_{z z}$ and $K_{x y}=K_{y z}=K_{z x}$ for the introduced thin-walled RVEs, which arise from their three-fold rotational symmetry about their diagonals. Equation (2.9) also predicts a linear relation between the relative wall thickness and the effective thermal conductivity. Figure 2.14 compares the prediction of Eq. (2.9) with the results of 3D standard mechanics homogenization for some of the thin-walled RVEs. Since the discrepancies between the aforementioned methods are highest for 0.1 relative wall thickness, the homogenization results for other relative thicknesses are not presented. As shown in Fig. 2.14, the predicted results by Eq. (2.9) show good agreement with the 3D homogenization. However, because the heat flow and thermal resistance normal to each RVE face are neglected, small $K_{x x}$ overprediction can be seen as if the results are slightly shifted to the right.

(a)

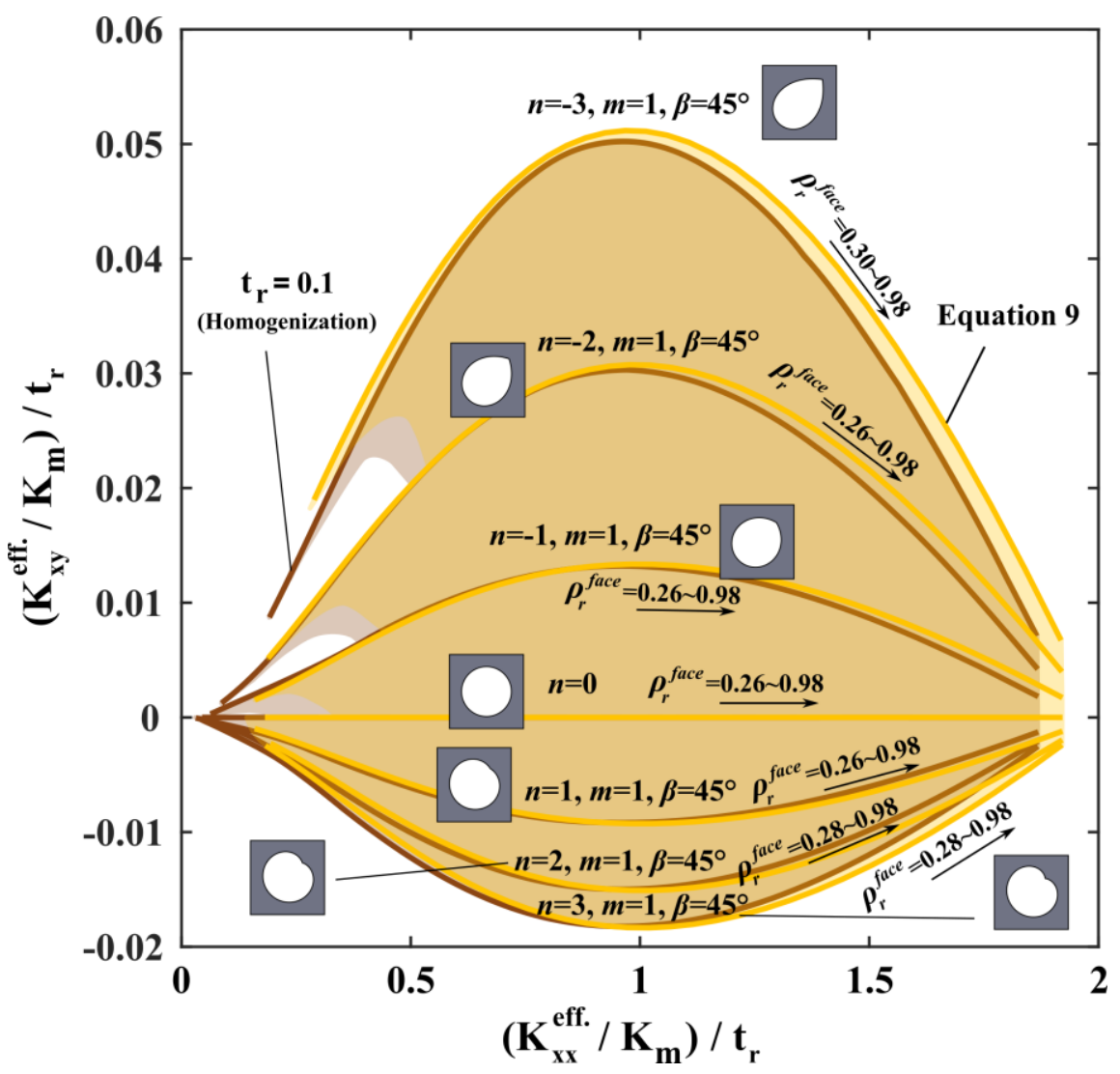




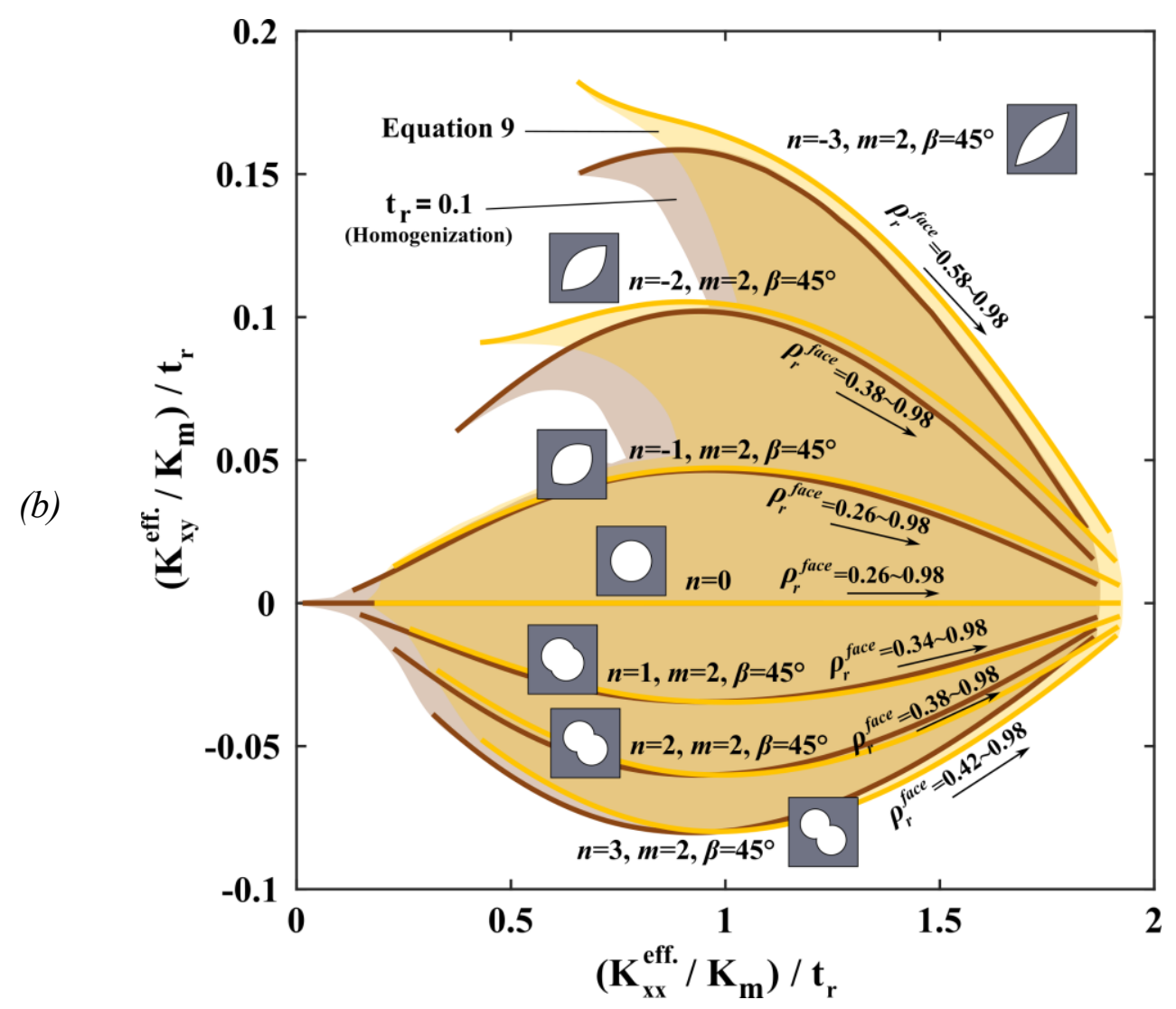

Figure 2.14: Effective $K_{x x}$ and $K_{x y}$ normalized by the base material's thermal conductivity times the relative wall thickness, for the thin-walled open lattices based on: (a) supershape parameter $m=1$ and (b) supershape parameter $m=2$. Only the face of the $3 D R V E$ in the $x-y$ plane is shown.

Although the superposition-based model shows good agreements with 3D standard mechanics homogenization, because it does not account for the influences of pore geometry of one face on the heat flow through the other faces, its prediction of the effective thermal conductivity of thinwalled open lattices might deviate noticeably from the 3D homogenization. An example is provided in Fig. 2.15, in which Eq. (2.9) predicts zero effective $K_{x y}$, while the actual value of this parameter changes with the relative density of 3D RVE for a supershape parameter set as $[n=-3$, $m=3, S=1, \beta=0$ ]. It should be noted that the assumptions made to neglect the heat transfer mechanisms other than conduction inside the matrix might lose their validity for lower relative densities of thin-walled open-cell lattices. 


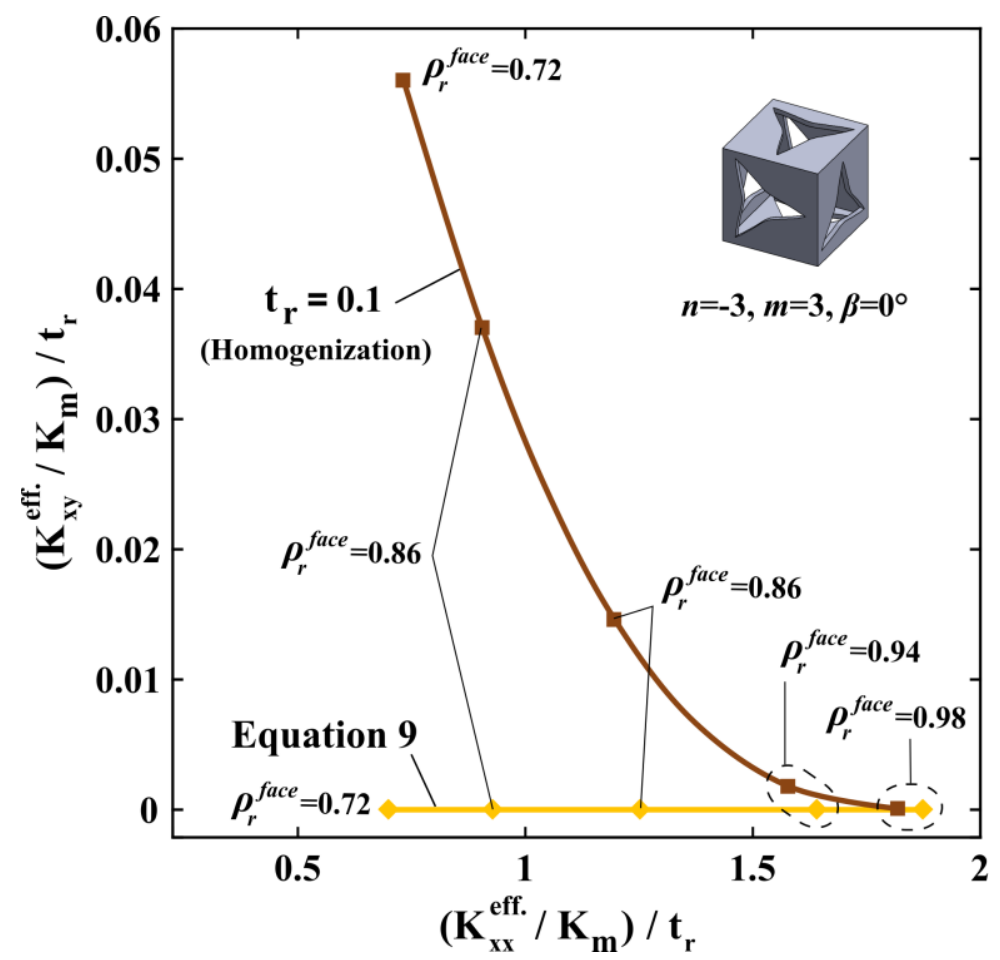

Figure 2.15: Deviation of the results of Eq. (2.9) from 3D standard mechanics homogenization for thin-walled open lattice with supershape parameters set as [n=-3, $m=3, S=1, \beta=0]$.

To show the versatility of the achievable effective thermal conductivity of thin-walled open lattices, their thermal conductivities are compared with P, D and G Shellular materials. The computational analysis shows that unlike most of the considered 2D and 3D architected cellular metamaterials, P, D and G Shellular materials are thermally isotropic and thus only one value is reported in Figs. 2.16 and 2.17. The G, D and P Shellular materials (when $f=0$ ) show almost equal thermal conductivity at each relative density, a phenomenon that is in agreement with the findings reported elsewhere [40]. Curve fittings of the effective thermal conductivities of these Shellular materials are provided in Table 2.4 (with the coefficient of determination $\mathrm{R}^{2}>0.9999$ ). As the power-law exponents for the relative density are all close to unity, it can be inferred that for relative densities below 0.25 , the thermal conductivity of G, D and P Shellular materials varies almost linearly with relative density. 


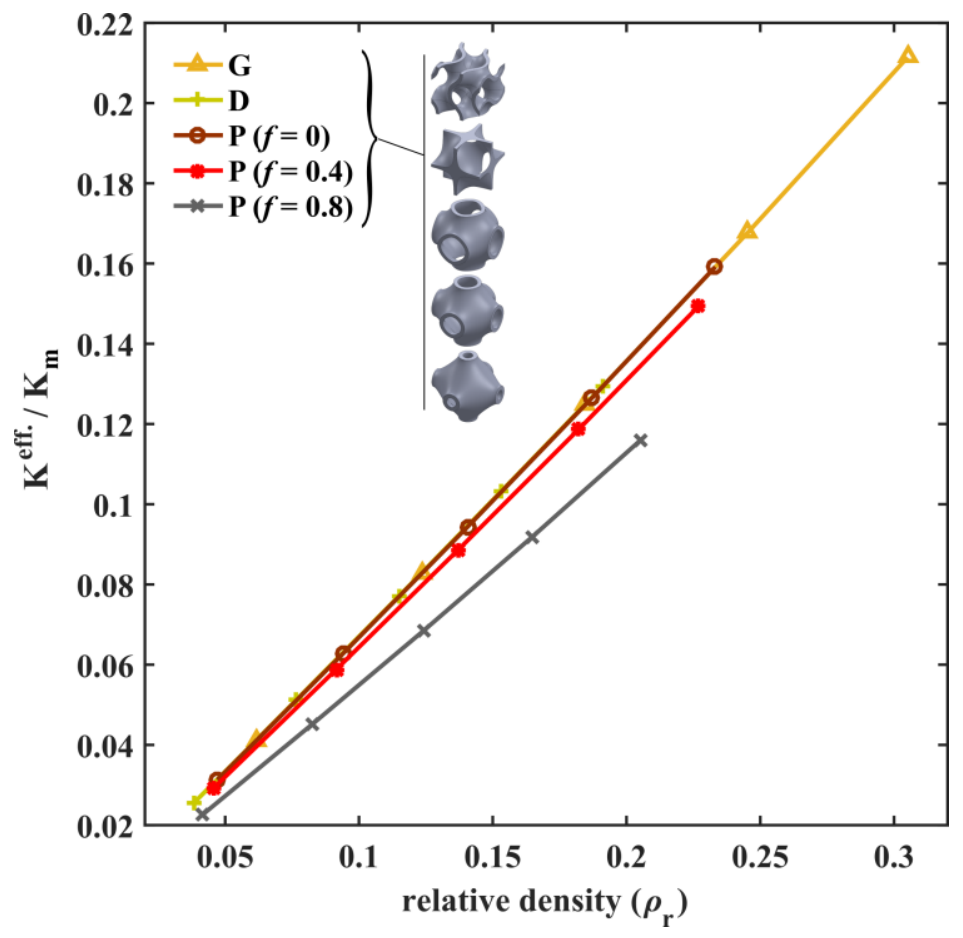

Figure 2.16: Effective thermal conductivity of Shellular materials, normalized by thermal conductivity of the solid matrix, with respect to relative density.

Table 2.4: Curve fittings of the normalized effective thermal conductivity of $P, D$ and $G$ Shellular materials in the form of $K^{\text {eff }} / K_{m}=C_{0}\left(\rho_{r}\right)^{n}$.

\begin{tabular}{|c|c|c|c|c|}
\hline \multirow{2}{*}{$\begin{array}{c}G \\
(f=0)\end{array}$} & \multirow{2}{*}{$\begin{array}{c}\text { P family } \\
(f=0)\end{array}$} & $f=0$ & $f=0.4$ & $f=0.8$ \\
\cline { 3 - 5 } & & $f=0)$ & $0.5808\left(\rho_{r}\right)^{1.0219}$ \\
\hline $0.7060\left(\rho_{r}\right)^{1.0219}$ & $0.6853\left(\rho_{r}\right)^{1.0089}$ & $0.6941\left(\rho_{r}\right)^{1.015}$ & $0.6700\left(\rho_{r}\right)^{1.0157}$ & 0.580 \\
\hline
\end{tabular}

Figure 2.17a shows the normalized effective thermal conductivity of P, D and G Shellular materials (with $f=0$ ) with respect to their relative wall thickness. As shown in this figure, for the same relative thickness, type $\mathrm{G}$ has the highest thermal conductivity among the three Shellular materials, while type D has the lowest thermal conductivity. Having the same unit cell dimensions, the G surface has more surface area than the other two Shellular materials, meaning that type G Shellular material has the highest relative density among the three Shellular materials for the same relative thickness. Considering relative density as one of the most influential parameters on the effective thermal conductivity, it is then reasonable for type G Shellular material to have higher 
effective thermal conductivity than P and D Shellular materials. With the same logic, as type D has the smallest surface area among the three selected surfaces, it has the lowest relative density for the same thickness and thus the smallest relative thermal conductivity. The effect of $f$ parameter on the overall thermal conductivity of type P Shellular materials is also examined. As shown in Fig. 2.16 and Fig. 2.17b, for the same relative density or the same relative thickness, increasing $f$ decreases the effective thermal conductivity of type $\mathrm{P}$ Shellular materials. This can be justified by the change in the spatial distribution of the material inside each cell. In other words, when $f=0$, more material is involved in the effective heat transfer throughout the Shellular medium compared to $f=0.8$.

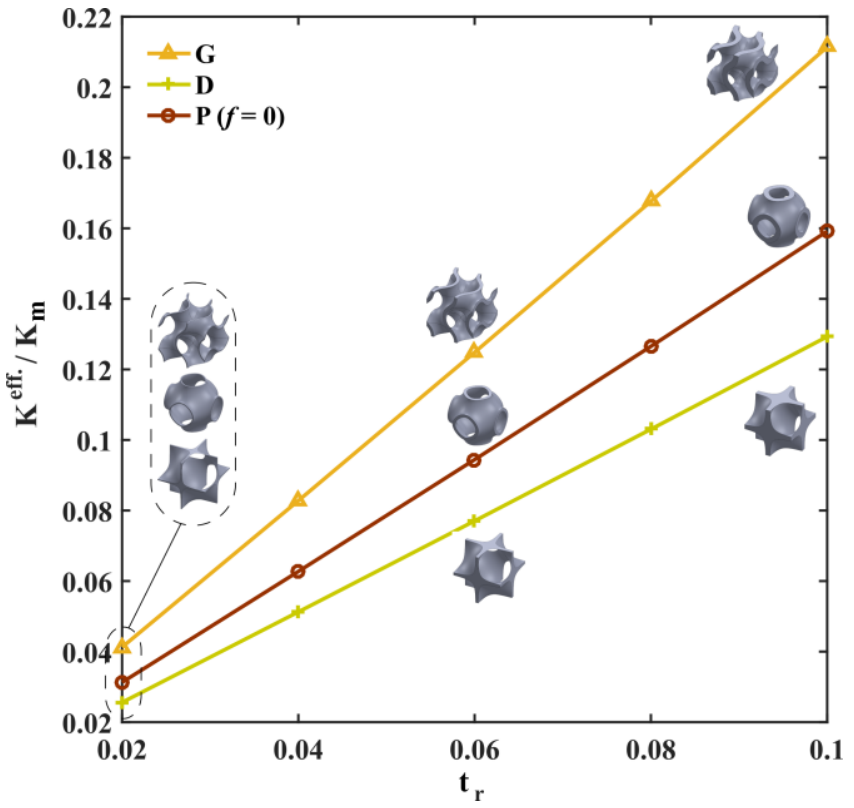

(a)

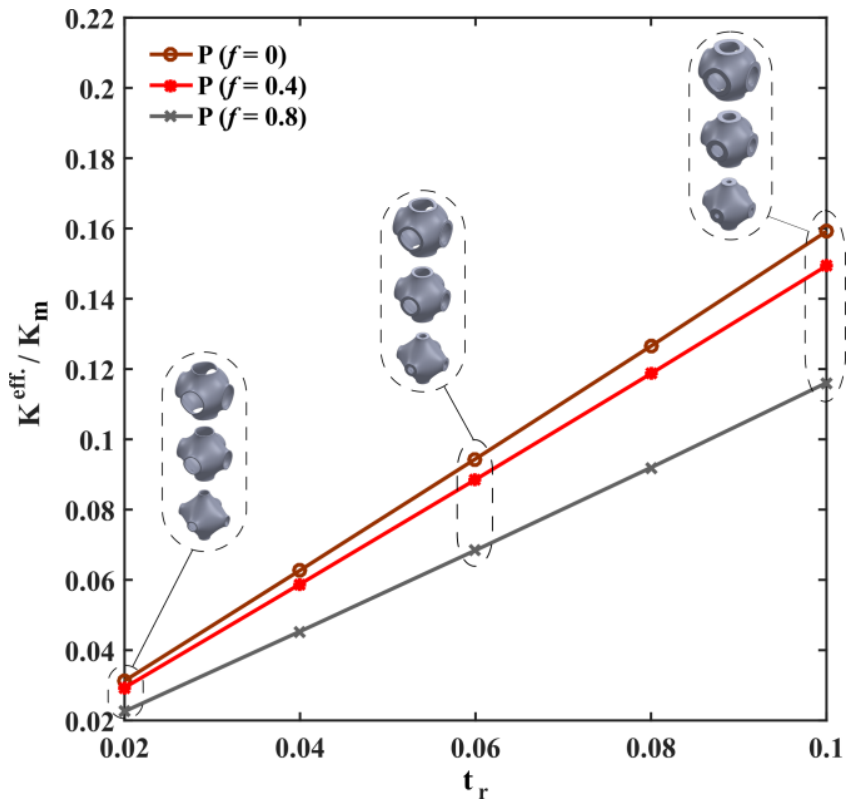

(b)

Figure 2.17: Normalized effective thermal conductivity of Shellular materials with respect to relative thickness: (a) $G, D$ and $P$ Shellular materials with $f=0$, and (b) P Shellular materials with different $f$ values.

Figure 2.18 presents the plots of $K_{1}^{e f f}-K_{2}^{e f f}$ for all 2D and 3D RVEs introduced in this paper. To compare the thermal conductivity of the 2D supershape, 3D thin-walled open lattices, and selected Shellular materials, $K_{1}^{e f f}$ and $K_{2}^{\text {eff }}$ are selected as the maximum and minimum principal effective thermal conductivity, respectively. It should be mentioned that for a 3D RVE made by thickening of a 2D supershape RVE, the effective out-of-plane thermal conductivity 
$\left(K_{z z}^{e f f}\right)$ follows the rule of mixtures since there is no topological change or heat transfer obstruction through thickness. The relation between $K_{1}^{e f f}, K_{2}^{e f f}$ and $K_{3}^{\text {eff }}$ for thin-walled open lattices with different $m$ and $n$ values is presented in Appendix 2.A4 (Fig. 2.A.4).

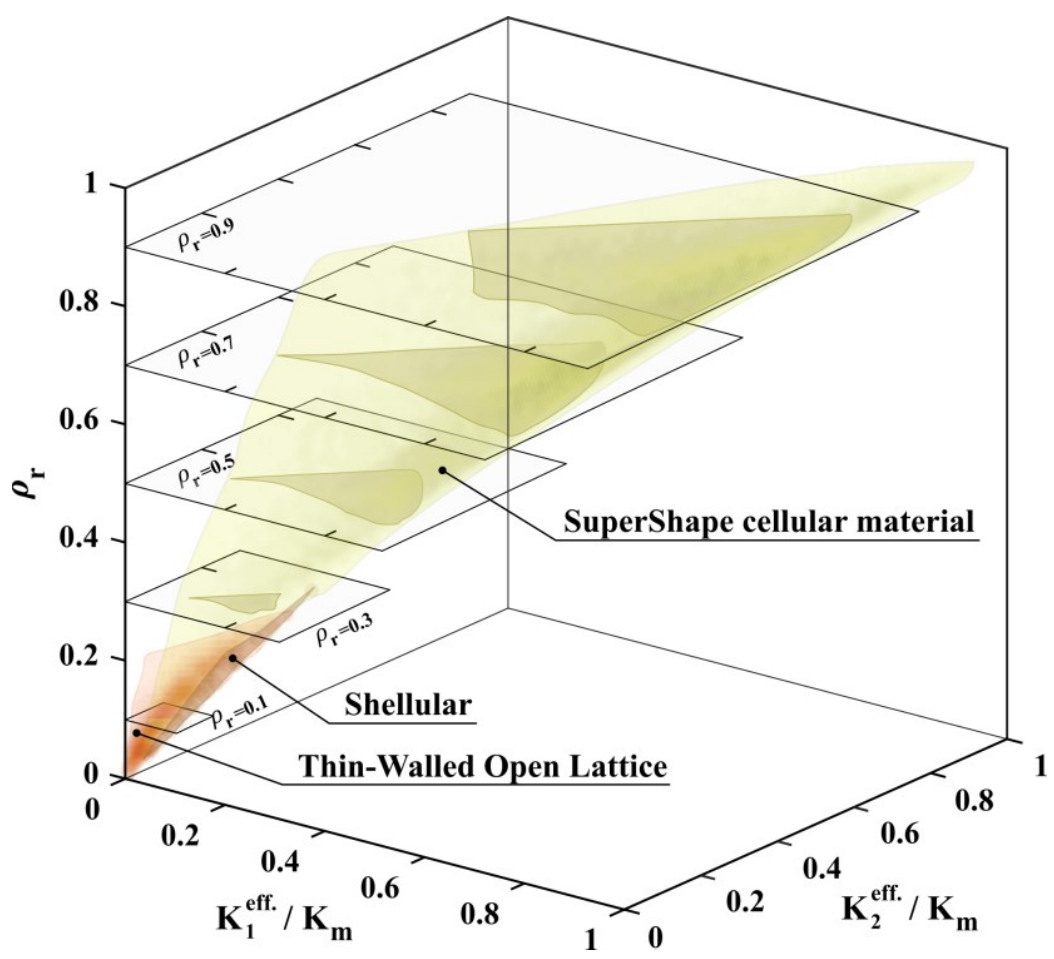

Figure 2.18: Maximum vs. minimum effective principal thermal conductivity of $2 D$ supershape cellular, 3D thin-walled open lattices, and Shellular materials for different relative densities.

Using a MarkerBot Z18, a fused deposition modelling (FDM) 3D printer, the manufacturability of the architected cellular metamaterials is verified. Figure 2.19 shows representative 3D printed unit cells made by layer-by-layer deposition of molten polylactic acid (PLA) using a $0.4 \mathrm{~mm}$ nozzle size, layer thickness around $0.1 \mathrm{~mm}$ and with 3D support structures where the part has overhang beyond $65^{\circ}$. Some common FDM 3D printing defects, such as rough surfaces, warpage, and shifted layers, are visible which can add extra thermal resistance when a 3D printed part is attached to another solid part. On the other hand, internal defects, such as internal delamination of layers and imperfect bonding, may significantly affect the overall thermal conductivity of the 3D printed part itself. While Fig. 2.19 confirms FDM 3D printing as a possible manufacturing process to produce cellular metamaterials, more precise additive manufacturing 
techniques, like stereolithography (SLA) or selective laser sintering (SLS), can be considered to reduce the manufacturing defects for the production of architected metamaterials.

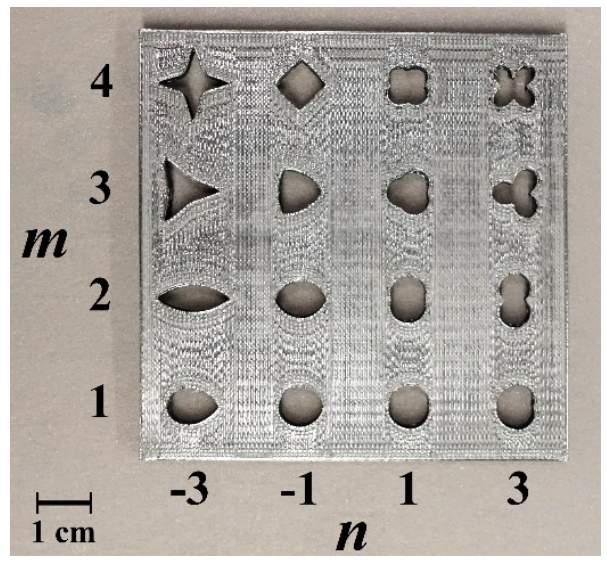

(a)

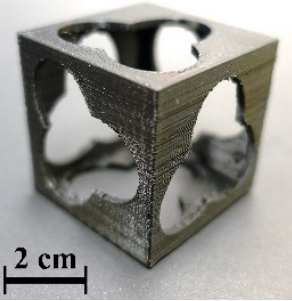

(b)

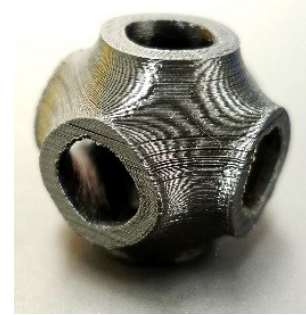

(e)

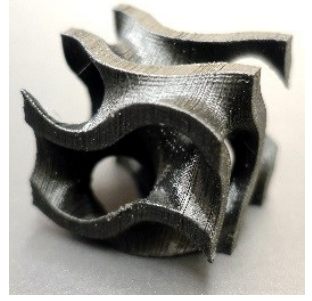

(c)

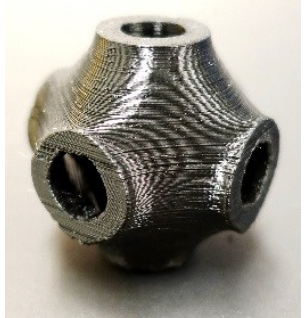

(f)

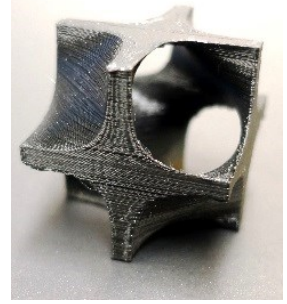

(d)

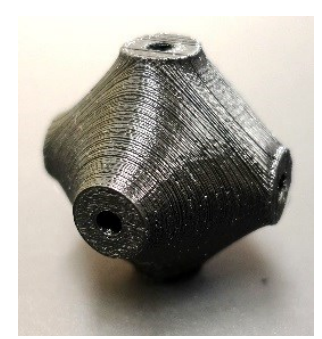

(g)

Figure 2.19: FDM 3D printed unit cells of some $2 D$ and $3 D$ RVEs presented in this paper: (a) 2D supershape RVEs, (b) Thin-walled open lattice, (c) Gyroid or G type Shellular $(f=0)$, (d) Diamond or D type Shellular $(f=0)$, (e) Primitive or P type Shellular with $f=0$, $(f)$ P type Shellular with $f=0.4$ and $(g) P$ type Shellular with $f=0.8$. All $3 D$ RVEs are $4 \times 4 \times 4 \mathrm{~cm}^{3}$.

\subsection{Functionally Graded Cellular Materials}

To explore the effect of tailoring the pore architecture across cellular metamaterials on tuning the thermal flow, topology and cell relative density of supershape pores can be graded according to the concept of functionally graded cellular materials (FGCMs) [59, 60]. Among all the possibilities, only the effect of pore rotation angle variation on the heat flux and temperature distribution is investigated here. Specific cell arrangements with similar pore shape but with different rotation angles are investigated to maximize or minimize the heat flow passing through the top $\left(\mathrm{Q}_{\mathrm{t}}\right)$ and right $\left(\mathrm{Q}_{\mathrm{r}}\right)$ edges of the cellular media while controlling the maximum temperature $\left(\mathrm{T}_{\max }\right)$ within these advanced cellular metamaterials. 


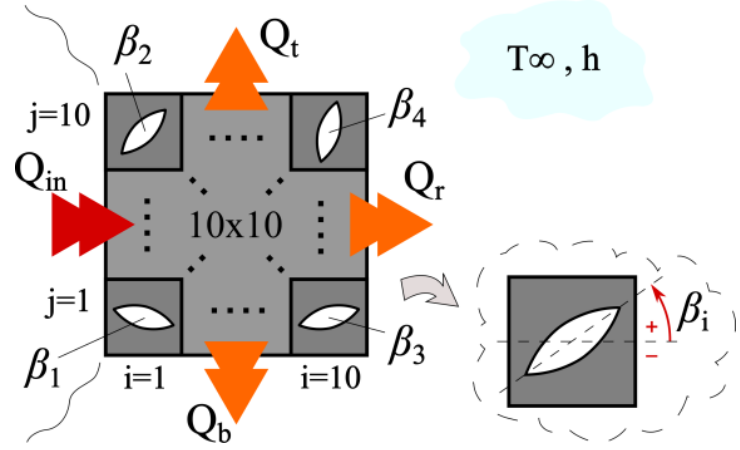

Figure 2.20: Schematic representation of an FGCM subjected to thermal loading.

As illustrated in Fig. 2.20, a $1 \mathrm{~m} \times 1 \mathrm{~m}$ 2D architected FGCM, consisting of $10 \times 10$ cells, is considered and $100 \mathrm{~W}$ heat is applied on the left side which leaves the cellular medium from the other three edges through heat convection with an ambient temperature of $0^{\circ} \mathrm{C}$ and a heat convection coefficient of $1 \mathrm{~W} / \mathrm{m}^{2} \mathrm{~K}$ (close to free convection in dry air). The base material of the FGCM is assumed isotropic with a thermal conductivity of $1 \mathrm{~W} / \mathrm{mK}$ (such as some non-technical ceramics). The topology of the empty pores within the FGCM is formed by the following sets of parameters in the supershape formula: $n=-2, m=2, S=1.5, \rho_{r}=0.8$. The pore angles of rotation $\left(\beta_{i}\right)$ at the four corners of the FGCM medium are independently tailored between $-90^{\circ}$ to $90^{\circ}$ with $\Delta \beta_{i}=22.5^{0}$. The rotation angle of the pores within the FGCM is linearly interpolated by:

$$
\begin{aligned}
& \beta(i, j)=\frac{\beta_{4}-\beta_{3}-\beta_{2}+\beta_{1}}{\left(n_{y}-1\right)\left(n_{x}-1\right)}(i-1)(j-1)+\frac{\beta_{3}-\beta_{1}}{\left(n_{x}-1\right)}(i-1)+\frac{\beta_{2}-\beta_{1}}{\left(n_{y}-1\right)}(j-1)+\beta_{1} \\
& \left(1 \leq i \leq n_{x}, 1 \leq j \leq n_{y}\right)
\end{aligned}
$$

where $n_{x}$ and $n_{y}$ are the number of cells in the $x$ and $y$ directions, respectively. Here, $n_{x}=n_{y}=$ 10.

The numerical results for the heat flux at the top $\left(\mathrm{Q}_{\mathrm{t}}\right)$ and right $\left(\mathrm{Q}_{\mathrm{r}}\right)$ edges, as well as the maximum temperature within the FGCM ( $\left.\mathrm{T}_{\max }\right)$, are illustrated in Fig. 2.21a, 2.21b and 2.21c, respectively. As a side note, nine cases exist where all the pores have the same rotation angle within the medium, representing periodic architected cellular materials that are not graded. Numerical results for these architected cellular cases are also separately presented in Fig. 2.22. 


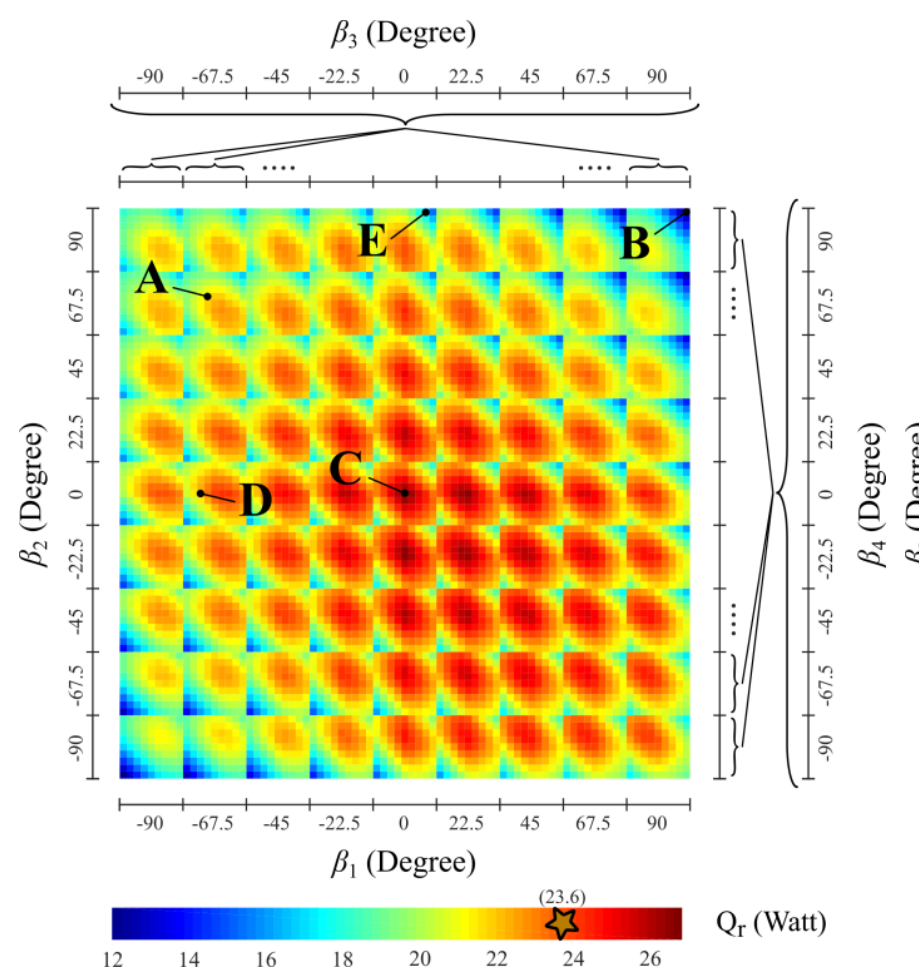

(a) $\beta_{3}$ (Degree)

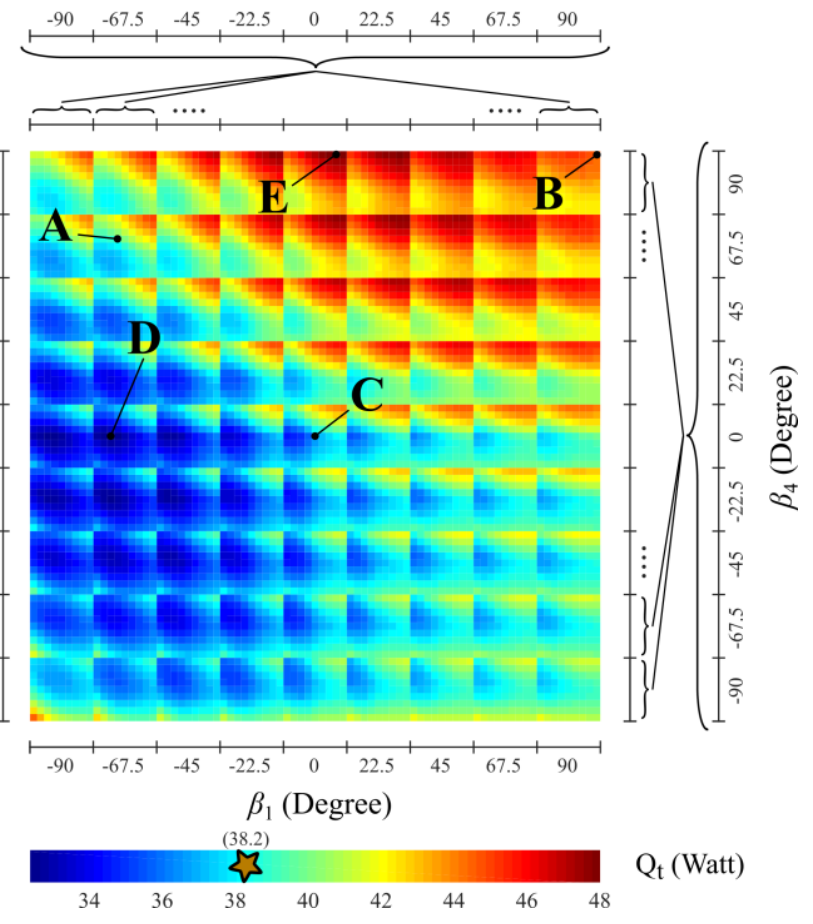

(b)

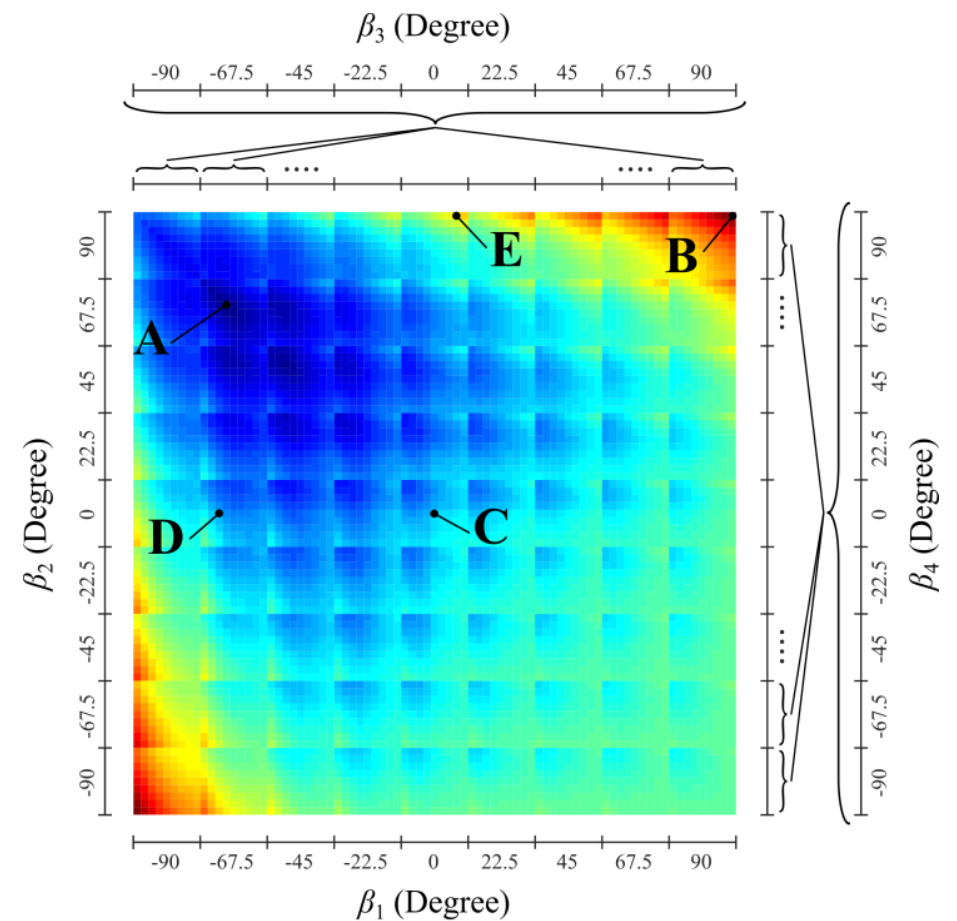

$\sum^{(82.2)}$

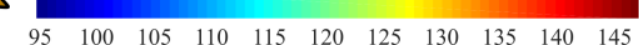

Temperature

$\left({ }^{\circ} \mathrm{C}\right)$

(c) 
Figure 2.21: Numerical simulation results of the square FGCM introduced in Fig. 2.20 for different $\beta_{1}$ to $\beta_{4}$ values. (a) Heat flow rate through the right edge ( $Q_{r}$ ), (b) Heat flow rate through the top edge $\left(Q_{t}\right)$, and (c) Maximum temperature throughout the FGCM. Stars on the color bars indicates $Q_{r}, Q_{t}$ and $T_{\max }$ for the solid part under the same boundary conditions. For points A E, please refer to Table 2.5 .

Within the FGCM, each pore can be considered as a wall guiding the heat to flow along its elongation direction, preventing it from transferring in the normal direction. Using this analogy, having all pores with zero rotation angle $\left(\beta_{1}=\beta_{2}=\beta_{3}=\beta_{4}=0^{\circ}\right)$ would guide more heat towards the right edge creating the case having maximum $\mathrm{Q}_{\mathrm{r}}$ (point $\mathrm{C}$ in Fig. 2.21). Similarly, when all the pores are $90^{\circ}$ rotated $\left(\beta_{1}=\beta_{2}=\beta_{3}=\beta_{4}=90^{\circ}\right.$ or $\left.-90^{\circ}\right)$, more heat is being blocked from going towards the right side resulting in the minimum $\mathrm{Q}_{\mathrm{r}}$ (point $\mathrm{B}$ in Fig. 2.21). For these two cases, the FGCM is symmetric about the $x$ axis. Therefore, as $\mathrm{Q}_{\mathrm{t}}$ and $\mathrm{Q}_{\mathrm{b}}$ are equal, they do not correspond to minimum and maximum $\mathrm{Q}_{t}$; but correspond to the minimum and maximum of $\mathrm{Q}_{t}+\mathrm{Q}_{b}$ (which is equal to $100-\mathrm{Q}_{\mathrm{r}}$ ). In other words, to maximize $\mathrm{Q}_{t}$ instead of minimizing $\mathrm{Q}_{\mathrm{r}}$, summation of $\mathrm{Q}_{\mathrm{r}}$ and $\mathrm{Q}_{b}$ shall be minimized. This can be achieved by arranging the pores in directions that maximize the heat flow towards the top edge, while minimizing it in the other two directions. Points $\mathrm{E}$ and $\mathrm{D}$ in Fig. 2.21 represent the maximum and minimum achievable $\mathrm{Q}_{\mathrm{t}}$. Introducing voids (or pores) into a solid media decreases its local thermal conductivity, which in turn translates into an increase in the maximum temperature. To control the increased maximum temperature, heat should encounter minimum resistance in its flowing direction towards the top, right and bottom edges. The functionally graded cellular arrangement corresponding to the minimum increase in $T_{\max }$ is indicated by point A in Fig. 2.21. Results for points A to E are summarized in Table 2.5. 


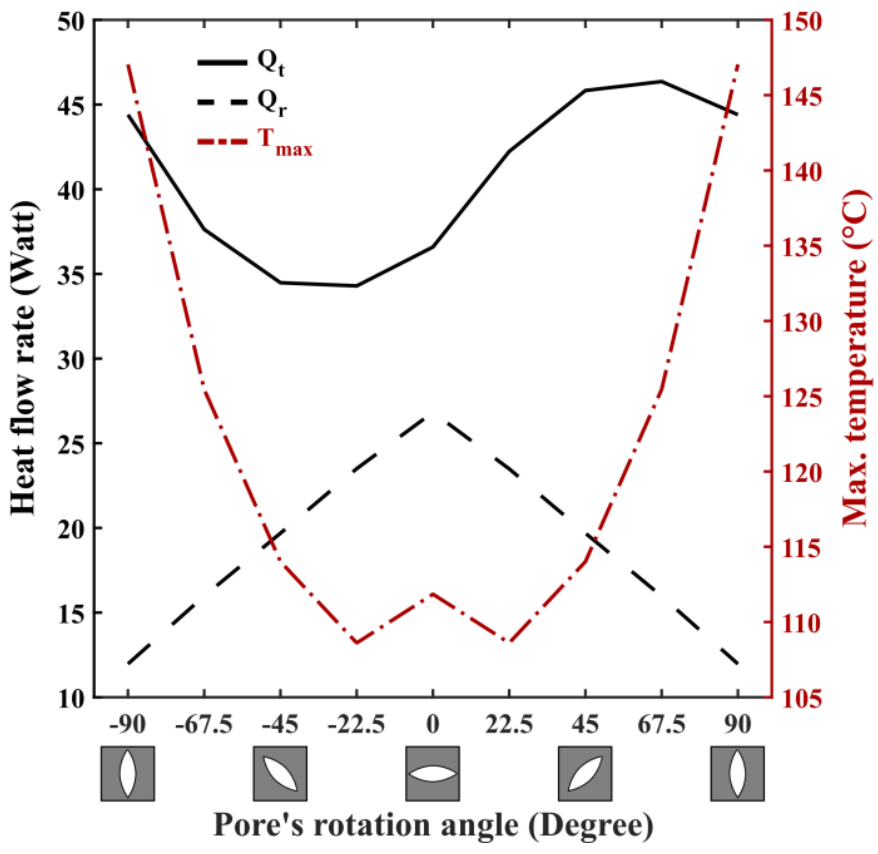

Figure 2.22: Heat flow rates through the top and right edges together with the maximum temperature as a function of pore's rotation angle, in the part made by uniform cellular materials $\left(\beta_{1}=\beta_{2}=\beta_{3}=\beta_{4}\right)$.

To demonstrate how tailoring the pore topology can tune the thermal performance of architected cellular media, numerical thermal results are compared with a corresponding fully solid medium. It can be seen that while the weight of both functionally graded and uniform cellular media is $20 \%$ lower than their solid counterparts, $\mathrm{Q}_{\mathrm{t}}$ can be increased by more than $25 \%$ using graded pore angles, compared to a $21 \%$ increase achievable by uniform cellular materials. As presented in Table 2.5, when minimizing the heat flux through the top edge of a cellular medium is of design interests, using an appropriate FGCM makes it possible to decrease $\mathrm{Q}_{\mathrm{t}}$ by $15 \%$, while a uniform cellular material can only reduce $\mathrm{Q}_{\mathrm{t}}$ by at most $10 \%$. In addition, it is possible to limit the unavoidable rise of the maximum temperature within the architected cellular medium and have $\mathrm{T}_{\max }$ equal to $115 \%$ of those of the solid medium value using the graded pore angles, while for uniform cellular media minimum $\mathrm{T}_{\max }$ is $32 \%$ higher than the solid medium. 
Table 2.5: Temperature contours of architected and graded cellular media along with the minimum and maximum values for $Q_{r}, Q_{t}$ and $T_{\max }$. The results of a solid medium are also provided as a baseline for comparison.

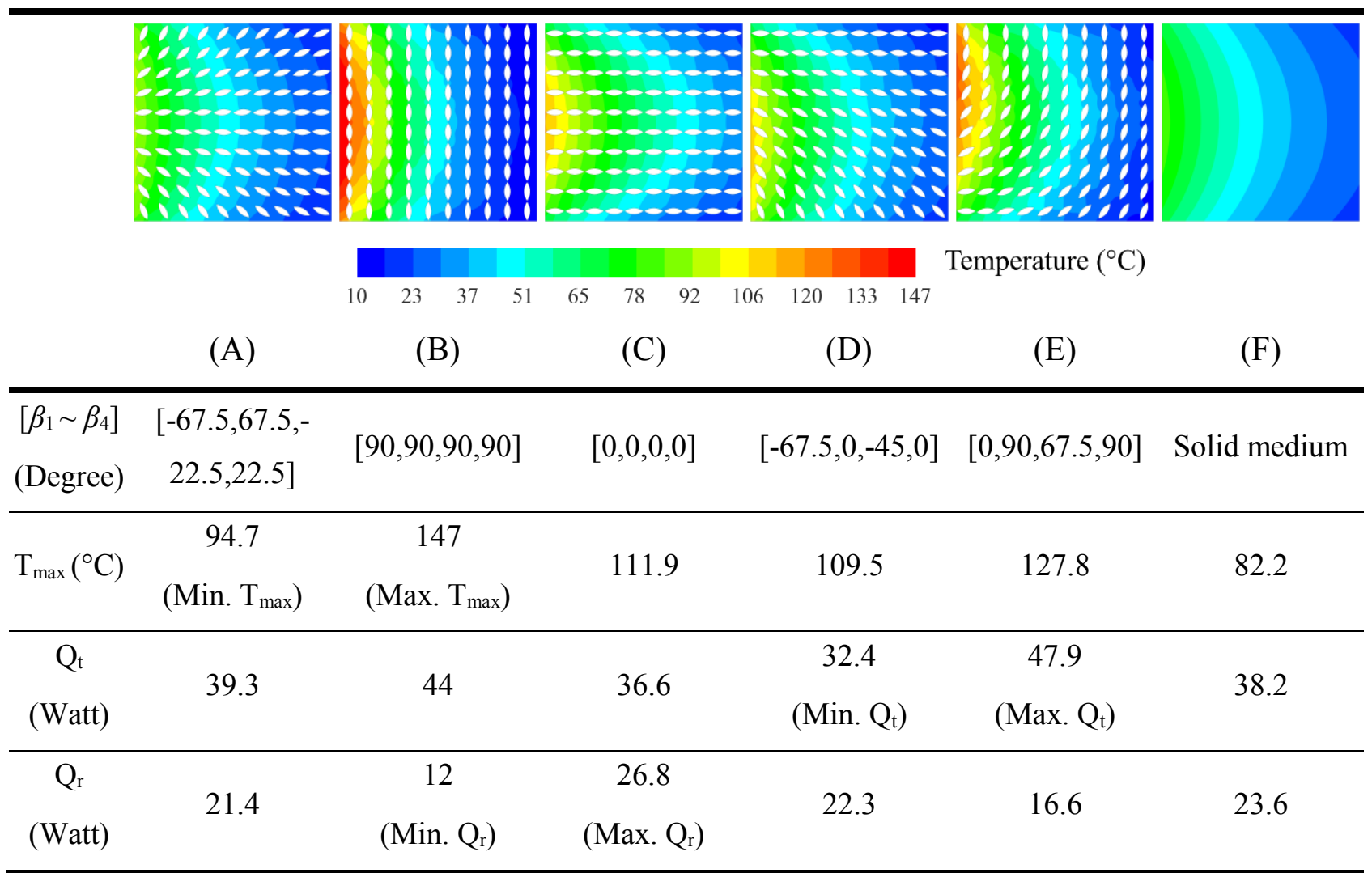

This section focused on the effect of grading pore rotation angles throughout an FGCM on the thermal flow and temperature distribution inside the material to show the advantages that architected FGCMs can offer for designing advanced materials to control/tune heat flow in desired directions and limiting the maximum temperature within cellular media. For structural applications of architected cellular metamaterials where thermal and mechanical properties are both crucial, in addition to the pore's rotation angle, other topological parameters of the cell microarchitecture (e.g., pore topology, relative density, elongation and even material composition) can also be spatially tailored throughout the FGCMs to achieve the highest multifunctional efficiency, for example in lightweight, structurally durable and highly-efficient thermal insulators. 


\subsection{CONCLUding REMARKS}

Theoretical and computational methodologies have been conducted in this paper for predicting the effective thermal conductivity of architected cellular metamaterials. We firstly examine the validity of neglecting the thermal conductivity of air inside the pores of cellular materials by determining their effective thermal conductivity using derived closed-form expressions and numerical homogenization. We then used computational prediction of standard mechanics homogenization for analyzing the effect of cell microarchitecture on effective thermal conductivity of 2D cellular metamaterials with supershape pore architectures of different pore shape, scaling and rotations. The results presented a wide range of achievable anisotropic effective thermal conductivity for different relative densities. While the results in this paper and in the available literature are mostly focused either on the effective thermal conductivity in the $x$ and $y$ directions, or the effective principal conductivities, we also explored the importance of offdiagonal terms in the thermal conductivity tensor using a case study. Then, the thermal conductivity of novel lightweight thin-walled open lattices, introduced based on 2D supershape pores, was examined. A mathematical model based on superposition was derived to calculate the effective thermal conductivity of thin-walled open lattices using the properties of 2D cellular materials with supershape pores. Comparing the predictions of this model with the numerical homogenization revealed that pores of one side of the cell alters the heat transfer through the other sides, further highlighting the importance of the cell architecture on the effective thermal conductivity of cellular materials. Different TPMS-based Shellular lightweight materials were also analyzed to compare their thermal conductivity with those of thin-walled open lattices. For the range of relative density investigated $\left(\rho_{r}<25 \%\right), \mathrm{G}, \mathrm{D}$ and $\mathrm{P}$ type Shellular materials with $f=0$ were found to have similar homogenized thermal conductivities. For the P type Shellular family, increasing the $f$ value from 0 to 0.8 decreased the effective thermal conductivity. Finally, using a case study in 2D, it was shown how the concept of architected functionally graded cellular materials can be used to tune the heat flow and temperature within a cellular medium. While the methodology presented in this paper sheds light on the engineering of thermal conductivity of

advanced cellular metamaterials, manufacturable by additive manufacturing technology, this study needs to be extended to also consider the other properties of cellular materials such as stiffness, electrical conductivity and permeability. This information will enable designers to develop engineered lightweight multifunctional structures in multiple length scales. 


\section{ACKNOWLEDGEMENT}

A.H. Akbarzadeh acknowledges the financial support of McGill University and Natural Sciences and Engineering Research Council of Canada (NSERC) through NSERC Discovery Grant RGPIN-2016-290471. The authors also acknowledge financial support from the Research Center for High Performance Polymer and Composite Systems (CREPEC).

\section{APPENDICES}

\section{A1 MODELING STEPS OF 2D RVES IN ANSYS APDL}

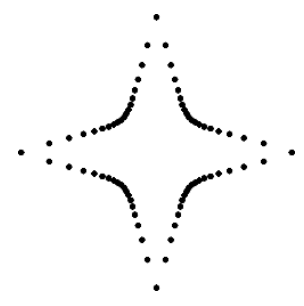

(a)

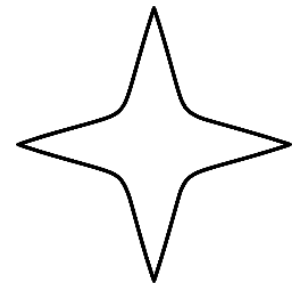

(b)

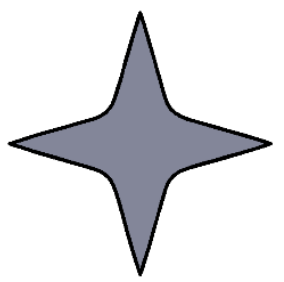

(c)

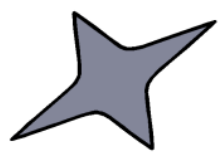

(d)

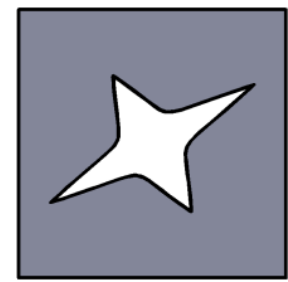

(e)

Figure 2.A.1: Modeling steps of 2D RVEs in ANSYS APDL: (a) keypoints, (b) spline segments passing through the keypoints, (c) pore area confined by the splines, without scaling and rotation, (d) pore area scaling and rotation and (e) subtraction of the pore area from a solid unit $R V E$.

\section{A2 STEPS FOR 3D MODELING OF A 3D SHELLULAR RVE}

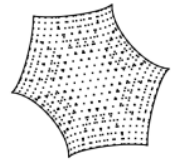

(a)

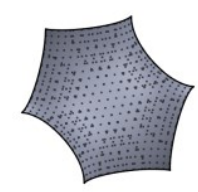

(b)

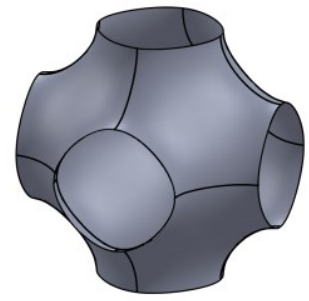

(c)

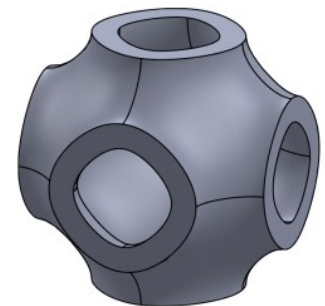

(d)

Figure 2.A.2: Steps for 3D modeling of a 3D Shellular RVE: (a) points and curves satisfying the level surface equation, (b) smooth surface passing through the points and curves to create the fundamental region, (c) using transformation to create the unit cell from the fundamental region and (d) adding thickness to the middle surface. 


\section{A3 VERIFICATION OF THE NUMERICAL HOMOGENIZATION RESULTS}

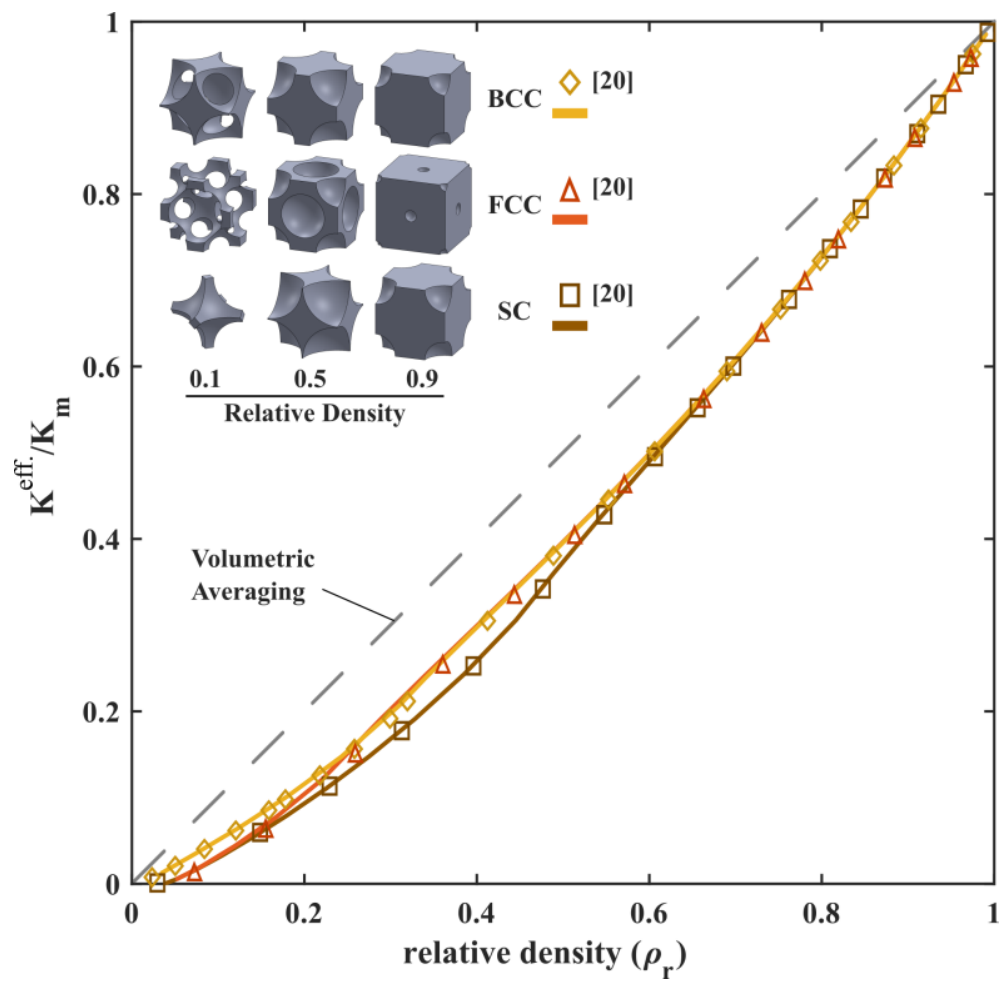

Figure 2.A.3: Obtained effective thermal conductivities based on the discussed numerical homogenization scheme (solid lines) and the values reported in [20] for the same periodic RVES

To verify and check the validity of the discussed numerical standard mechanics homogenization technique, the obtained effective thermal conductivities of periodic cubic RVEs with spherical pores in simple cubic (SC), face-centered cubic (FCC) and body-centered cubic (BCC) arrangements are compared with those presented in [20], calculated using a solver based on explicit jump immersed interface method (readers are referred to reference [20] for more details). Despite using different methods to calculate effective thermal conductivities, very good agreement between the results is demonstrated in the Fig. 2.A.3.

It should be noted that by increasing the pore size larger than a specific value, solid parts of the RVEs become isolated and the periodic material loses its continuity. As a result, the effective thermal conductivity lines do not cross the origin in Fig. 2.A.3. The minimum relative density of the CAD models for the analysis are about 0.04 for the SC and FCC arrangements and 0.02 for the BCC. 
2.A4 EFFECT OF $M$ AND $N$ ON THE RELATION BETWEEN EFFECTIVE PRINCIPAL THERMAL CONDUCTIVITIES OF THIN-WALLED OPEN LATTICES

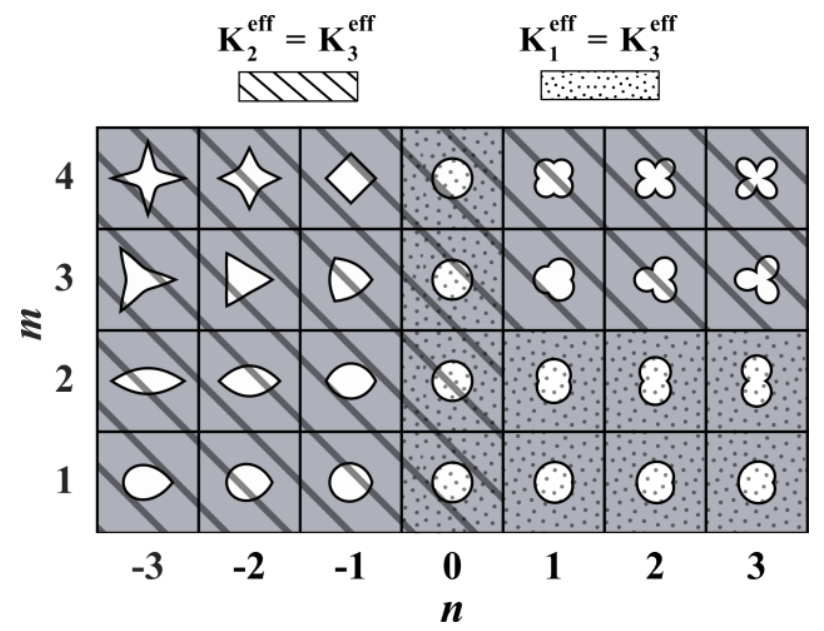

Figure 2.A.4: Relation between effective principal thermal conductivities of thin-walled open lattices for different $m$ and $n$ values of porous faces and $0^{\circ} \leq \beta \leq 90^{\circ}$.

As a result of the three folded rotational symmetry, the effective thermal conductivity tensor of thin-walled open lattices in $x-y-z$ coordinate system is presented only by two values $\left(K_{x x}^{e f f}\right.$ and $\left.K_{x y}^{e f f}\right)$ in the form of:

$$
K^{e f f}=\left[\begin{array}{lll}
K_{x x}^{e f f} & K_{x y}^{e f f} & K_{x y}^{e f f} \\
K_{x y}^{e f f} & K_{x x}^{\text {eff }} & K_{x y}^{\text {eff }} \\
K_{x y}^{\text {eff }} & K_{x y}^{\text {eff }} & K_{x x}^{\text {eff }}
\end{array}\right]
$$

Calculating the eigenvalues of this matrix gives the three effective principal thermal conductivities, one equals to $K_{x x}^{e f f}+2 K_{x y}^{e f f}$ and the other two equal to $K_{x x}^{e f f}-K_{x y}^{e f f}$. Therefore for $K_{x y}^{\text {eff }}>0$, the maximum $\left(K_{1}^{e f f}\right)$ and minimum $\left(K_{2}^{\text {eff }}\right)$ effective principal thermal conductivities are $K_{x x}^{e f f}+2 K_{x y}^{e f f}$ and $K_{x x}^{e f f}-K_{x y}^{e f f}$ respectively, and the third effective principal thermal conductivity $K_{3}^{\text {eff }}$ is equal to $K_{2}^{\text {eff }}$. For $K_{x y}^{\text {eff }}<0$ on the other hand, $K_{1}^{\text {eff }}=K_{3}^{\text {eff }}=$ $K_{x x}^{e f f}-K_{x y}^{e f f}$ and $K_{2}^{e f f}=K_{x x}^{e f f}+2 K_{x y}^{e f f}$. Figure 2.A.4 shows the relation between $K_{1}^{e f f}, K_{2}^{e f f}$ and $K_{3}^{e f f}$ for thin-walled open lattices. It is assumed that $K_{1}^{e f f}$ is the maximum and $K_{2}^{e f f}$ is the minimum effective principal thermal conductivity. 


\section{Connecting Statement}

Chapter 2 provided in-depth analysis of effective thermal conductivity of some $2 \mathrm{D}$ and 3D cellular architectures. As one of the findings, it is shown that with the introduction of supershape holes on the faces of a hollow cube, it would be possible to design cellular materials with a wider range of thermal conductivity compared to Shellular materials. In the next chapter, to expand the range of effective thermal conductivity of Shellular architectures, the possibility of systematically introducing holes on these materials is evaluated. It is shown that 2D holes in cellular arrangement can be mapped on a subsection of the TPMS using conformal mapping. Holes are then cut from this subsection and the rest of the TPMS unit cell is created using transformation of the perforated part. By thickening the unit cell of the perforated TPMS, the RVE of a conformally perforated Shellular material is built. Effective thermal conductivities of some architectures created by this method are investigated as case studies.

Chapter 3 is a part of ongoing studies on different properties of Shellular and perforatedShellular materials, in collaboration with Shahin Eskandari, Jiahao Shi, and Prof. Abdolhamid Akbarzadeh at $\mathrm{AM}^{3} \mathrm{~L}$ laboratory of McGill University. 


\section{Thermal Conductivity of Conformally Perforated Shellular Metamaterials}

\subsection{INTRODUCTION}

Using P, D and G-type Shellular architectures, low relative density cellular materials can be created. These structures consist of a shell-like solid phase that forms the architecture and divides the space into two disjoint sub-spaces (labyrinths) [39, 46, 61, 62]. As a TPMS is continuous, the interconnection between the two sub-spaces are cut by the solid material. Furthermore, there is no parameter in their mathematical model to change in order to modify their underlying architecture and subsequently their effective properties at a given relative thickness or relative density. To consider these points, Shellular architectures can be upgraded by introducing perforations on their surfaces. Adding holes, not only further reduces the relative density, but also makes the two disjoint sub-spaces interconnected, which in turn would further increase the permeability and open up the door to potential new applications in ultralight advanced structural materials, thermal insulation, tissue engineering scaffolds, catalyst supports, filtration and battery electrodes.

Introducing holes on the Shellular architecture can be done by any arbitrary approach, as long as the integrity of the solid structure is intact. Similarly, pore shape and arrangement as well as the number of holes (perforations) can also be adjusted as desired. In this article, inspired by the idea of hierarchical architectures [63], holes arranged in a 2D periodic cellular layout are selected to be mapped on and cut from the TPMS. The idea is to make a 3D Shellular architecture perforated based on a $2 \mathrm{D}$ cellular layout and investigate the relation between the properties of the resulting material and the two incorporated 2D and 3D cellular architectures. To keep the connection with the previous works (please refer to Chapter 2 of this thesis), 2D cells are selected to be square. Knowing that the cell shape and pore topology determine the effective properties of a cellular material, it is obvious that distortions through the mapping shall be kept as small as possible to have a simple and meaningful correlation between the properties of the 3D perforated Shellular material and the 2D cellular layout used for perforating the TPMS. However, mapping a 2D flat region to a 3D curved surface (and the reverse operation) unavoidably distorts it, which indicates the importance of selecting an appropriate mapping method. In the current manuscript, discrete conformal mapping $[64,65]$ is selected as an appropriate method for transformation between the $3 \mathrm{D}$ and 2D layouts. 
In the following sections, after reviewing the steps in CAD modeling of conformally perforated Shellular materials (abbreviated as CPSM), the effective thermal conductivity of selected CPSM is obtained using standard homogenization (Section 3.4). The aforementioned hypothetical relation between the properties of the underlying $2 \mathrm{D}$ and $3 \mathrm{D}$ architectures are then assessed. The manuscript is concluded with a short summary of the incorporated methods and major findings.

\subsection{CONFORMAL MAPPING}

Conformal mapping, also known as angle-preserving transformation, is a transformation that preserves local angles (magnitude and orientation), except at some probable points on the boundary, hence a 2D circle mapped on a 3D surface will look like a circle, or corners of a rectangle mapped on a $3 \mathrm{D}$ surface will still be $90^{\circ}$. Using this mapping method, although the $2 \mathrm{D}$ rectangular cells will unavoidably deform when mapped on the 3D surface, the area and angle distortions are tentatively kept to be minimum $[64,65]$.

In this research, mapping is done using the method provided in reference [64], in which a discrete triangulated surface is first mapped onto the unit disk by solving the Laplace equation, and then, the unit disk is mapped to the unit square by solving the generalized Laplace equation introduced in that reference. The height of the unit square is finally optimized to achieve conformality. The Riemann mapping theorem guarantees the existence of a conformal map from any simply-connected open surfaces to the unit disk and consequently a rectangle with unit width. For detailed discussion about conformal mapping, readers are referred to reference [64]. The resultant $2 \mathrm{D}$ rectangle's height depends on the choice of the corner points. While any point on the boundary of the 3D surface can be selected as a corner, it is preferable to choose points at which the boundary of the 3D surface has an angle closest to $90^{\circ}$. This would further decrease the area and angle distortion associated with mapping, especially on the boundary of the surface. Figure 3.1 shows the selected portion of the RVE of the Primitive, Diamond and Gyroid TPMS, denoted as fundamental region since the whole corresponding RVE can be constructed from this portion using rotation, translation or reflection transformations. 
(a)

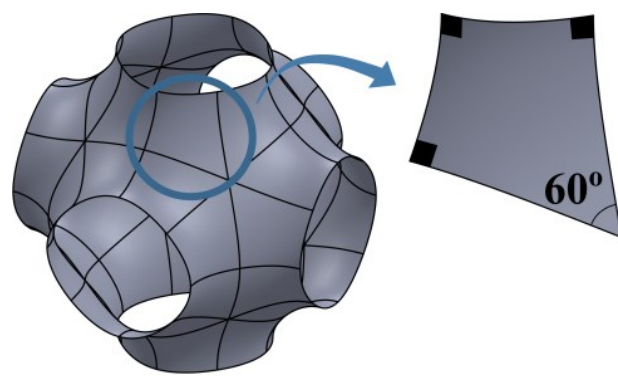

(b)

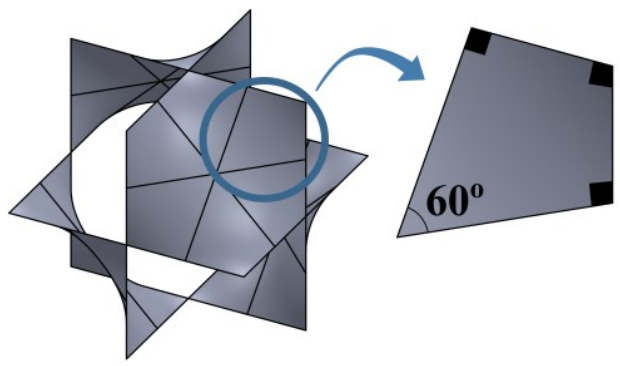

(c)

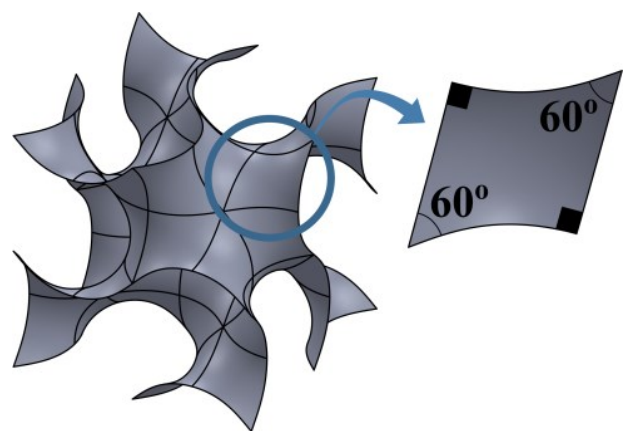

Figure 3.1: Fundamental regions of (a) Primitive, (b) Diamond, and (c) Gyroid TPMS.

By choosing corners of the fundamental regions shown in Fig. 3.1, for P and D surfaces the height of the mapped 2D rectangle is also equal to unity, allowing a $2 \mathrm{D}$ cellular layout made of square cells to be mapped on them. However, height of the conformally mapped fundamental region of the $\mathrm{G}$ surface is not unity, making it impossible to contain $n \times n$ square cells. Defining $a$ and $b$ as the longer and shorter edges of the mapped rectangle and $l_{a}$ and $l_{b}$ as their lengths, the aspect ratio $\left(l_{a} / l_{b}\right)$ of the 2D mapped fundamental region of G-type TPMS is found to be 1.134.

Alternatively for the case of G-type CPSM, instead of square RVEs, rectangular 2D cells can be mapped to the 3D fundamental region. Needless to say, dividing its width and height to the same number of cells $(n)$ would create small rectangular cells with the same aspect ratio. However, if the shorter edge $b$ is divided into $n$ segments (with segment's length $d b$ equals to $l_{b} / n$ ) and the longer edge $a$ is divided by the nearest integer number to $l_{a} / d b$, increasing $n$ to more than 5 would reduce the aspect ratio to smaller than 1.05 . The effect of further increasing the $n$ number on the aspect ratio of the rectangular cells is presented in Fig. 3.2. As shown in this figure, in general by increasing $n$, the aspect ratio gets closer to unity meaning that with small enough cells it would be possible to get practically square RVEs mapped to the Gyroid surface as well. 


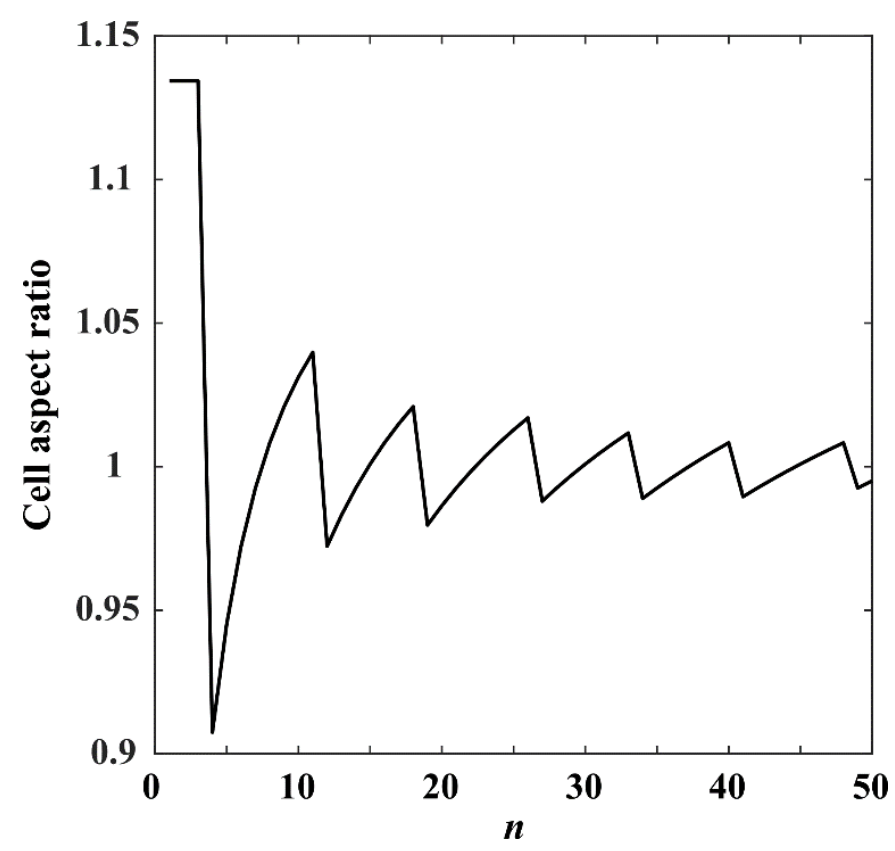

Figure 3.2: Effect of increasing the number of divisions on the smaller edge of the rectangular mapping of the fundamental region of G-type TPMS on the cell aspect ratio, while the longer edge is being divided by the nearest integer number to $l_{a} / d b$

Although increasing the number of cells with the aforementioned method seems like a promising method that enables mapping square cells on the G-type TPMS, it makes the CAD modeling and FEM used for homogenization computationally expensive. As a result, and to only focus on the perfectly square 2D RVEs similar to our previous works, G type CPSM is not included among the architectures analyzed in this manuscript.

\subsection{CAD MOdELLING}

Steps for CAD modeling of CPSM are presented in Fig. 3.3. The first step is to model the fundamental region of the selected TPMS. Here, the previously modeled RVEs of the TPMS (Section 2.2.3) are trimmed so that the remaining part is the fundamental region. Thereafter, saving the file to STL format creates the triangulated surface. Exporting high resolution STL file and having a finer triangulated surface would increase the accuracy of mapping. The STL file is then imported to MATLAB, and checked to remove probable duplicate entities. Next, using the method provided by [64] (briefly discussed in Section 3.2), it is conformally mapped to a 2D rectangular region. The $2 \mathrm{D}$ rectangle is then covered by the $2 \mathrm{D}$ unit cells with the desired pore topology, made 
by several points. To find the position of the corresponding points on the 3D surface, locations of the 2D points on each triangular facet are found with respect to the corners of that triangle, and knowing the corresponding triangular facet on the $3 \mathrm{D}$ triangulated surface, it is possible to calculate the location of each point mapped back to the 3D surface. After finding the corresponding locations of the points of the 2D pores on the 3D surface, these points are created in the original CAD model of the smooth fundamental region in SolidWorks. By connecting the points around each pore with one or more splines and cutting the surface with them, 3D pores are introduced to the surface. It is important to note that one closed spline is enough to create smooth pore topologies without a sharp corner (e.g., circle); however, to preserve the corners of pore shapes with sharp angles (e.g., square) multiple connected splines shall be used. Using linear, circular and mirror transformations of the perforated fundamental region, the unit cell of the perforated TPMS is made. Finally the assembly of at least $2 \times 2 \times 2$ of this unit cell is thickened and subsequently cut, to make the final unit cell of CPSM. Doing this, assures that the concluding RVE has flat sides and is periodic in three dimensions. To reduce the file size and ease the importing process of the CAD models into ANSYS (or COMSOL), generated RVEs are saved to Parasolid .x_t format.

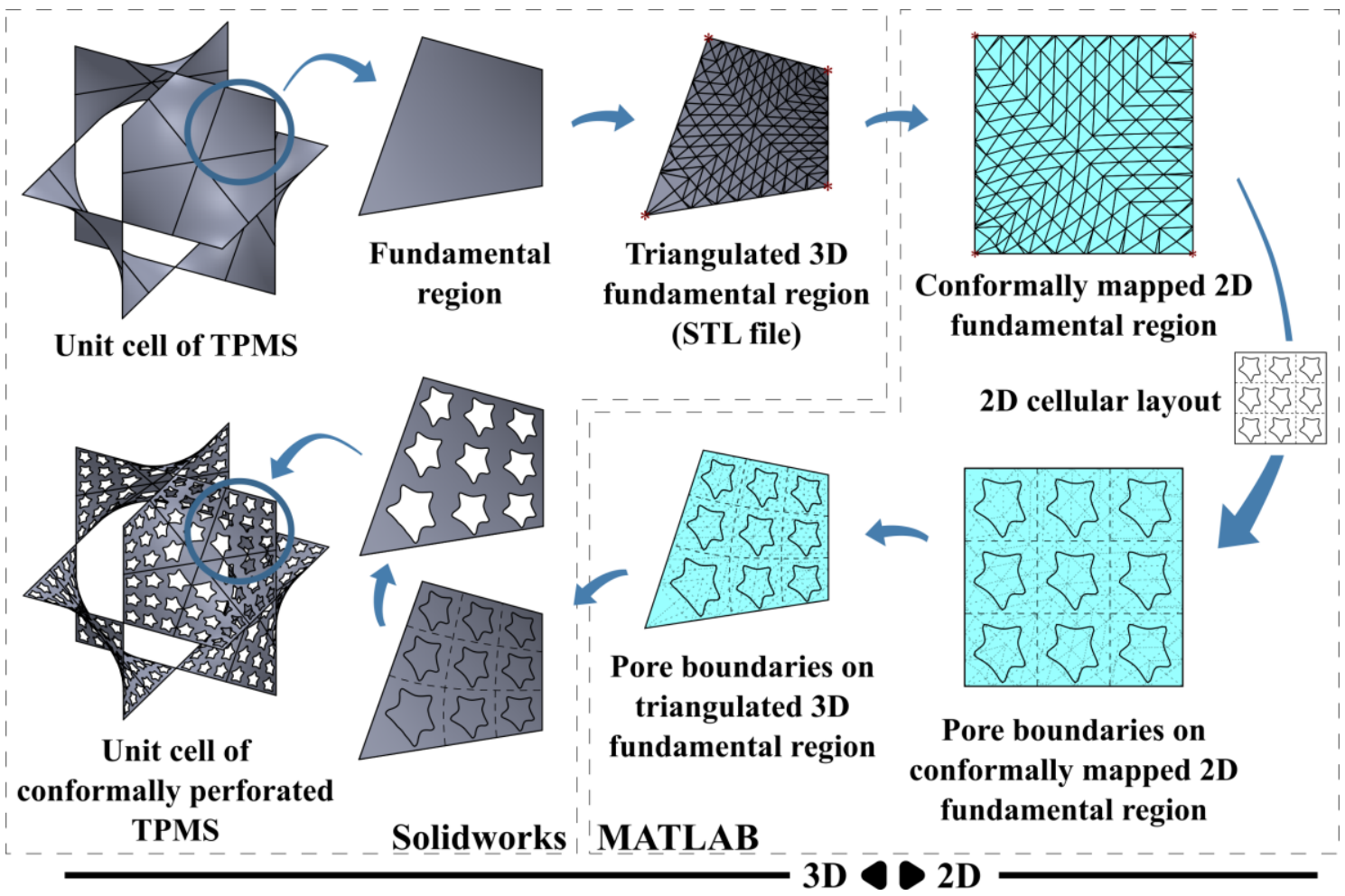

Figure 3.3: Steps for CAD modeling of conformally perforated TPMS. 
The manufacturability of CPSM is tested by 3D printing sample P, D and G-type CPSMs using a Makerbot Replicator FDM 3D printer and PLA fillament, with a 0.4mm nozzle size and $0.25 \mathrm{~mm}$ layer height setting. Images of the 3D printed samples are provided in Fig. 3.4. While some defect and imperfections are noticeable, the surface finish and overall quality of the part can be increased by fine tuning the 3D printing parameters which are out of the scope of the present study.

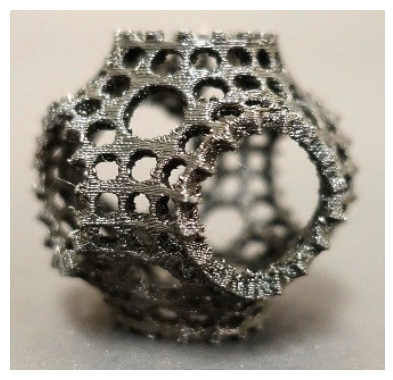

(a)

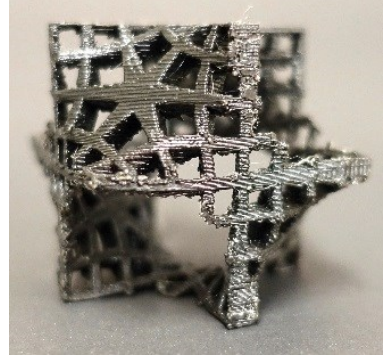

(b)

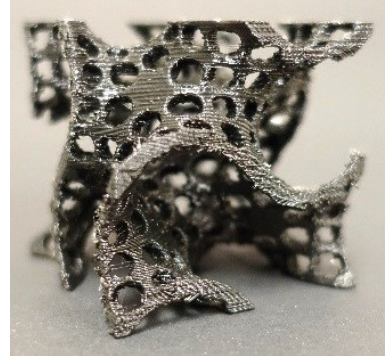

(c)

Figure 3.4: Sample FDM 3D printed CPSMs based on layouts of 2-by-2 2D cells of 0.5 relative density: (a) P-type CPSM with shifted semi-circular pores, (b) D-type CPSM with semi-square pores and (c) G-type CPSM with semi-circular pores.

\subsection{HoMOgENIZATION}

After modeling the RVEs of the CPSM, effective properties of the cellular material made from these architected cells can be predicted using homogenization. In this work, to accurately calculate the effective thermal conductivity of these metamaterials, periodic boundary conditions are applied to the RVEs and numerical standard homogenization [16] is employed.

In this method, it is assumed that under the same temperature gradient applied to the boundaries of the cells, temperature gradient at any point on the RVE of the cellular architecture $(\nabla T)$ and the corresponding point on the RVE of the equivalent homogenized material are related by a tensor, denoted as $M^{T}$. Knowing that the temperature gradient at any point on the RVE of the homogenized material is equal to the average temperature gradient on that cell $(\overline{\nabla T})$, the aforementioned relation can be mathematically written as $\nabla T=M^{T} \overline{\nabla T}$ for any point on the RVE of the cellular material. To calculate $M^{T}$ tensor, three independent unit thermal gradients are applied to the boundaries of the cubic RVE with unit dimensions: 


$$
\left(\begin{array}{l}
T(-0.5, y, z)-T(0.5, y, z) \\
T(x,-0.5, z)-T(x, 0.5, z) \\
T(x, y,-0.5)-T(x, y, 0.5)
\end{array}\right)_{i}=\left(\begin{array}{l}
1 \\
0 \\
0
\end{array}\right)_{i=1},\left(\begin{array}{l}
0 \\
1 \\
0
\end{array}\right)_{i=2},\left(\begin{array}{l}
0 \\
0 \\
1
\end{array}\right)_{i=3}
$$

where $i$ is the thermal loading case number, $x, y$ and $z$ are on the boundaries of the RVE and the center of the unit cubic RVE is assumed to be located at the origin.

Under each loading case Fourier heat conduction simulation is performed inside ANSYS APDL environment and the resulting thermal gradients at each element is calculated and subsequently stored using this FEA package. $M^{T}$ tensor is then constructed based on the stored thermal gradients. The effective thermal conductivity of the cellular material made from the chosen RVE is then calculated by:

$$
\bar{K}_{i j}=\frac{1}{V_{R V E}} \int K_{i k} M_{k j}^{T} d V_{R V E} \quad(i, j, k=1,2,3)
$$

in which, $\bar{K}_{i j}$ is the effective thermal conductivity tensor, $V_{R V E}$ represents the RVE volume, and $\mathrm{K}_{\mathrm{ik}}$ is the local thermal conductivity tensor.

\subsection{RESULTS AND DISCUSSION}

The main objective of this study is to investigate the possibility of upgrading Shellular materials' architecture by introducing a mapped layout of holes on them, and showing that with this upgrade it would be possible to tune their effective thermal conductivities. Figure 3.5 provides a variety of CPSM architectures, to demonstrate the potentials of the presented method for modeling CPSMs with any arbitrary pore shapes. While Fig. 3.5 shows that P, D and G-type CPSMs can be modeled using this method, in this manuscript only P-type CPSM with 2D square cells containing circular and square pores are considered to showcase and investigate the hypothetical relation between their effective thermal conductivities and those of their underlying 2D and 3D architectures. Characteristics of the selected underlying architectures and their effective properties, presented in Chapter 2, are listed in Table 3.1. 
(a)

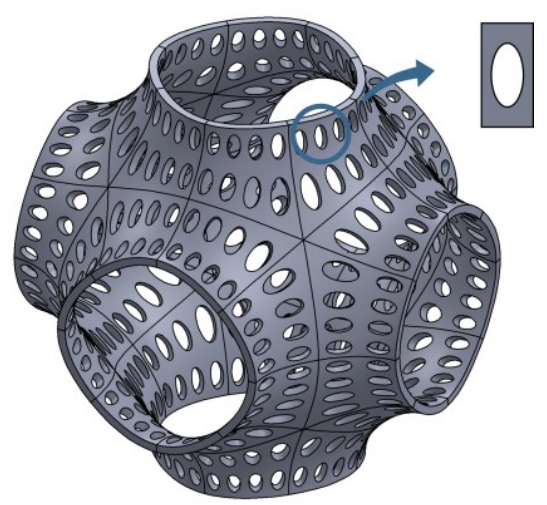

(c)

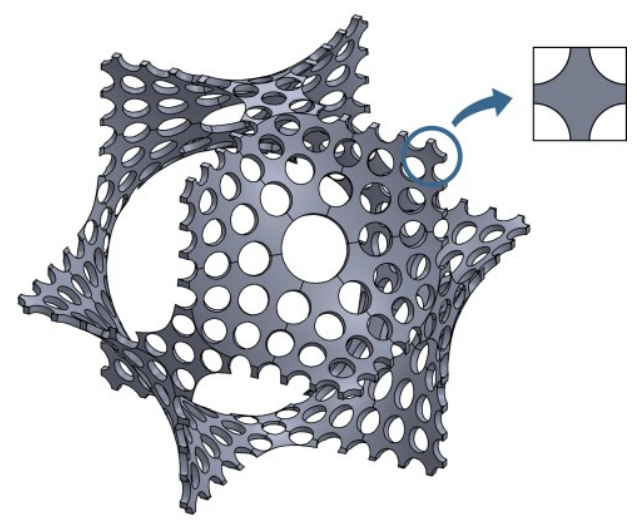

(b)

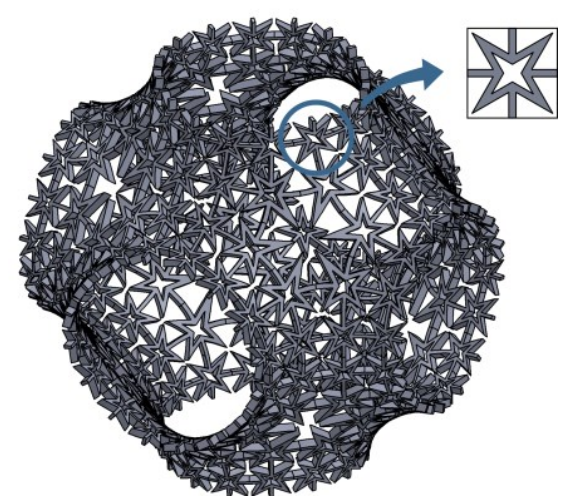

(d)

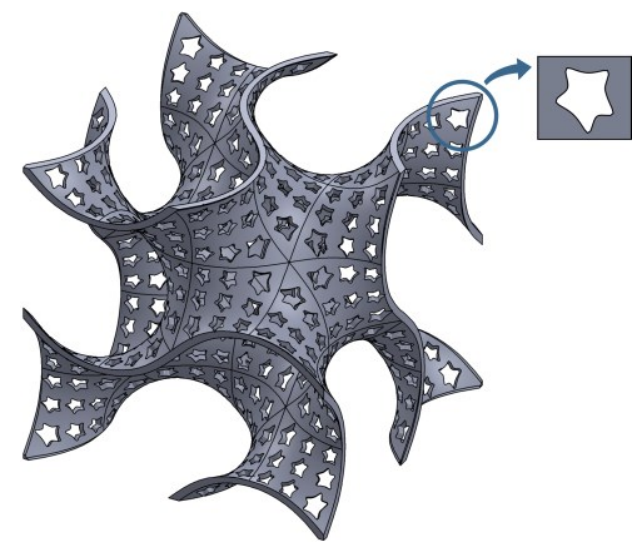

Figure 3.5: Representative CAD models of CPSMs based on: (a) P-type Shellular and 2D rectangular cell with an elliptic pore, (b) P-type Shellular and $2 D$ re-entrant square cell, (c) Dtype Shellular and 2D square cell with shifted circular pore, and (d) G-type Shellular and 2D rectangular cell with an arbitrary pore shape. Relative thicknesses of all models are 0.02.

To merely investigate the contribution of the base material's heat conduction to the overall thermal conductivity of the CPSM, gaseous inclusion is neglected (that is, vacuumed pore or pore with low thermal conductivity gases at low pressure). It is also assumed that the material is approximately at ambient condition and temperature difference throughout the CPSM is small, so that the convection and radiation heat transfer mechanisms can be ignored [54-57]. Under these assumptions, effective thermal conductivity of the selected P-type CPSM are numerically calculated using the standard homogenization method discussed in Section 3.4. 
Table 3.1: Characteristics of the underlying architectures of the selected P-type CPSM

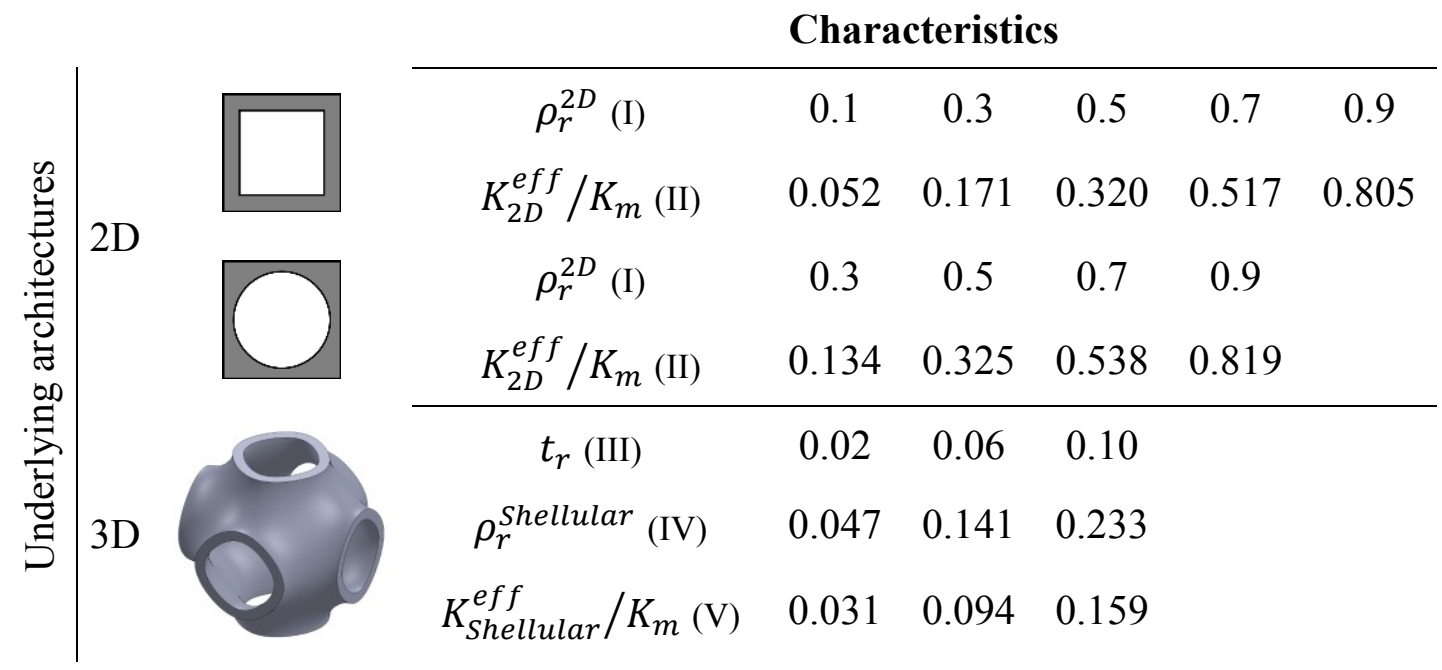

I. Relative density of the underlying $2 \mathrm{D}$ architecture

II. Effective thermal conductivity of the underlying 2D architecture, normalized by thermal conductivity of the base material

III. Relative thickness (equals to Shellular's thickness divided by the RVE's length)

IV. Relative density of the underlying 3D Shellular architecture

V. Effective thermal conductivity of the underlying 3D P-type Shellular architecture, normalized by thermal conductivity of the base material

With the existing rotational symmetries, it is evident that both of the selected 2D architectures and the 3D P-type Shellular materials are thermally isotropic. Assuming that the area distortion through the mapping process is small, cell topology and relative density before and after mapping would be similar, suggesting that the in-plane conductivity of the 2D and their corresponding mapped 3D cells shall be approximately equal and the slightly deformed version of the 2D square cell shall also be thermally isotropic. The area distortion inside the fundamental region is found to be small, though it may not be small close to the boundaries (e.g., as shown in Fig. 3.1a, there is a $60^{\circ}$ corner on the fundamental region of the $\mathrm{P}$ surface, which is being mapped to a $90^{\circ}$ corner). However, by increasing the number of mapped cells on the fundamental region, the ratio of the more distorted cells close to boundary with respect to the inner cells will decrease, meaning that the overall thermal property of the fundamental region should converge to that of the 2D cell. With the considerations about the area distortion and assuming that within the thickened surface the heat flow component normal to the shell at any point is significantly smaller than the heat flow tangent to it, it could be theorized that with enough number of mapped cells, effective thermal conductivity 
of the selected CPSMs should reach to the multiplication of the effective thermal conductivity of the underlying $2 \mathrm{D}$ and $3 \mathrm{D}$ architectures. To assess this theory, using the homogenization results and the data provided in Table 3.1, the effect of increasing the number of holes on the effective thermal conductivity of the selected CPSM is examined in Fig. 3.6. In this regards, as previously shown in Fig. 3.3, 2D $n \times n$ layouts of circular holes are mapped on the fundamental region of the P-type TPMS and cut to make the CPSM.

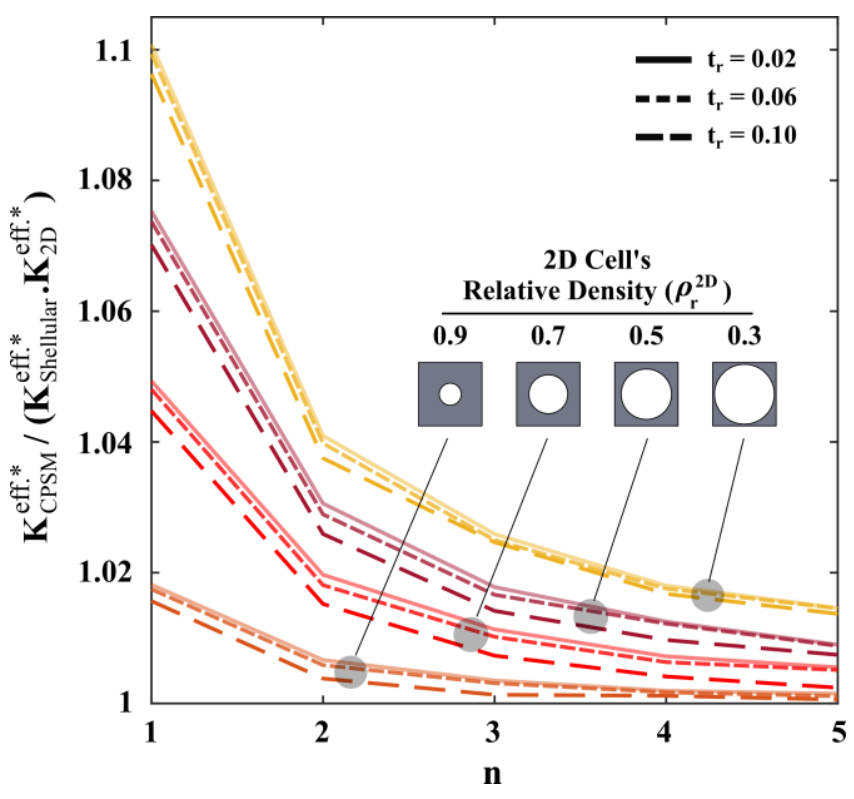

(a)

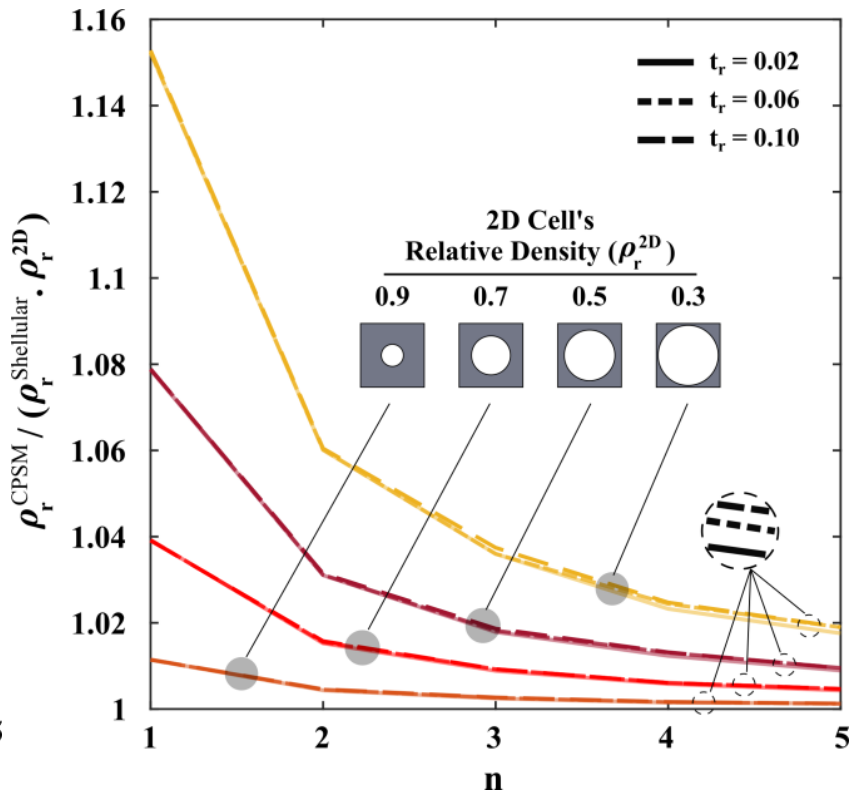

(b)

Figure 3.6: Dependency of properties of the selected CPSMs (described in Table 3.1) on the number of mapped holes on the fundamental region of the underlying P-surface, (a) normalized effective thermal conductivity and (b) relative density. Effective thermal conductivities denoted with a star are normalized by the conductivity of the base material

As shown in Fig. 3.6, numerical results support the theory mentioned in the previous paragraph, in which by increasing the number of circular holes that are conformally mapped to the fundamental region of the P-surface, the overall thermal conductivity of the CPSM converges to that of a hypothetical P-type Shellular with the same thickness made out of a cellular material with square cells and circular holes. Having only $3 \times 3$ cells conformally mapped on the fundamental region, the error associated with the predictions of the theory with respect to the detailed homogenization method is found to be less than 3 percent. With this in mind, to reduce the 
computational cost, effective thermal conductivity of a CPSM based on a $3 \times 3$ cellular layout of 2D square cells with square pores and P-type Shellular with 0.02 relative thickness are also obtained using standard mechanics homogenization to compare with the anticipated values from the multiplication of the effective thermal conductivity of the underlying 2D and 3D architectures.

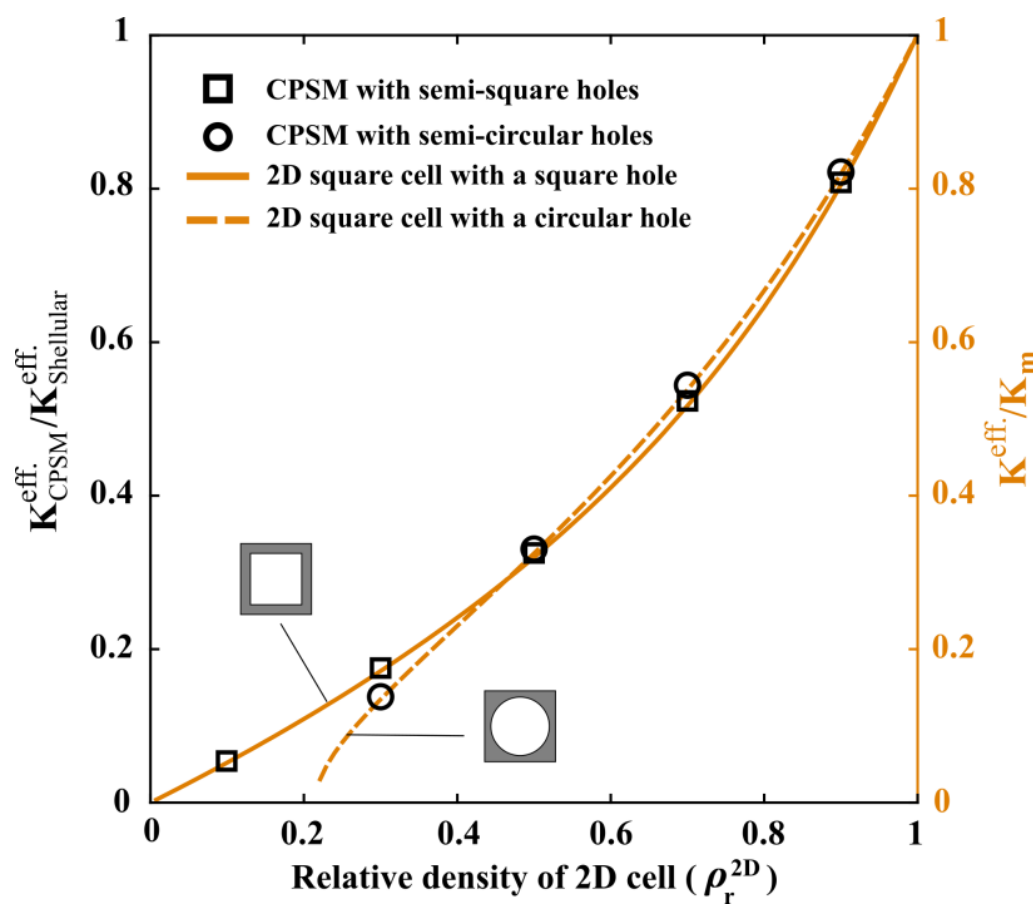

(a)

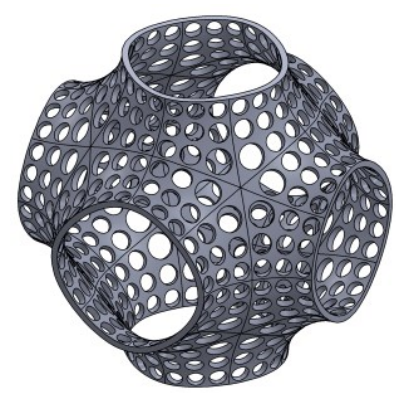

(b)

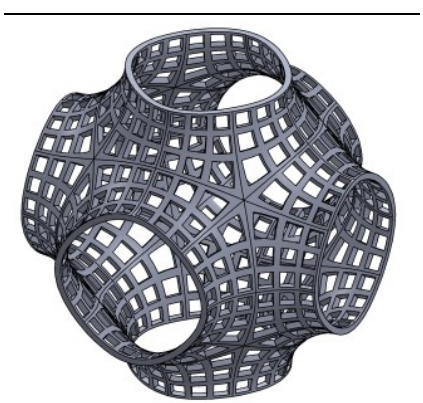

(c)

Figure 3.7: (a) Overall thermal conductivity of P-type CPSMs (with 0.02 relative thickness) with respect to relative density of its underlying $2 D$ architectures; (b) and (c) show the RVEs of the Ptype CPSM with semi-circular and semi-square holes with $2 D$ relative densities equal to 0.5 .

As presented in Fig. 3.7, effective thermal conductivity of the CPSMs normalized by the effective thermal conductivity of the underlying P type Shellular architecture with 0.02 relative thickness, is plotted with respect to the relative density of the underlying $2 \mathrm{D}$ architectures. Results shown in Fig. 3.7 clearly show that the normalized effective thermal conductivities of CPSMs for both semi-circular and semi-square perforation cases match the homogenization results for $2 \mathrm{D}$ cells with perfect circular and square pores, further confirming the validity of the theory and its assumptions for at least when the underlying 2D cellular architecture of the CPSM is thermally isotropic. 


\subsection{CONCLUding REMARKS}

Deeming the fundamental regions of a 3D TPMS as a deformed 2D surface, it is hypothesized that it should be possible to warp a 2D cellular layout to make the fundamental region of the $3 \mathrm{D}$ perforated TPMS. To minimize the cell distortion, conformal mapping (which locally preserves the angles through the transformation) is selected to transform the $2 \mathrm{D}$ cellular layout into the $3 \mathrm{D}$ fundamental region of TPMS. The conformally perforated Shellular architectures created by this methodology are imported to ANSYS in order to perform numerical standard mechanics homogenization and to accurately predict their effective thermal conductivities. Case studies on the P-type CPSM with 2D layout of cells with circular and square pores, show that by increasing the number of cells on the 2D layout thermal properties of the CPSM change as if the mapped 2D cells on the 3D surface are perfectly periodic. This will significantly reduce the time required to obtain the effective thermal properties of this type of ultralight architectures.

The objective of this work was to show the possibility of adjusting the thermal conductivity of Shellular materials by introducing holes on them. Further studies are required to investigate the overall thermal conductivity of different CPSMs with $2 \mathrm{D}$ cells that may not be necessarily thermally isotropic. 


\section{CONNECTING Statement}

In Chapter 3, a methodology is presented to imprint a 2D sub-structure on Shellular materials which is used to create semi-hierarchical metamaterials with $2 \mathrm{D}$ cells as the base material and a 3D Shellular as the 3D architecture. A few case studies are performed to investigate the effective thermal conductivity of the new ultralight perforated Shellular materials using standard mechanics homogenization. Although compared to the Shellular material, the underlying 2D cellular structure is at a smaller length scale, both of them are assumed to be at continuum level and the heat transfer simulations performed on them are based on Fourier heat conduction. While this assumption is generally effective at micro to macro scales, material behaviour at the nanoscale can be quite different. In Chapter 4, effective properties of macroscopic analogues of some truss-like cellular nano-architectures based on carbon nanotube are obtained using standard mechanics homogenization and are compared with the properties of their nanoscale counterparts predicted by molecular dynamics (MD) simulation.

Chapter 4 is a detailed report of the contribution of the author of this thesis in the following manuscript published in the Carbon journal:

- C. Zhang, A.H. Akbarzadeh, W. Kang, J. Wang, A. Mirabolghasemi, "Nano-architected metamaterials: Carbon nanotube-based nanotrusses", Carbon, 2018. 131: p. 38-46 


\section{Thermal Conductivity of FCC ANd SC Periodic Truss Materials}

\subsection{INTRODUCTION}

Thanks to the ever growing computing power of modern computers, the idea of cellular materials has been recently brought to the nano meter dimensions to design cellular nano architectures at atomic length scales [23, 66, 67]. Studies on material at this size have shown that properties can be quite different compared to those of the same material at continuum level. Colloids of gold nano particles at different nano dimensions exhibiting different colors from red to blue [68], or extraordinary structural and thermal properties of carbon nano tubes [69-71] are two examples which clearly show significantly different material properties at the nanoscale compared to the normal properties seen at the macro scale. These dissimilarities are attributed to different physics governing the structural and thermal behavior of materials at different length scales [23].

In the present study, simplified macroscopic models of carbon nanotube-based nanotrusses with SC and FCC cellular architectures are created and while Diamond, as a material with properties comparable to those of carbon nanotube (CNT), is chosen as the base material for the cell architectures at the macro scale, their effective properties are obtained by performing standard mechanics homogenization on their representative unit cells under periodic boundary conductions. Effective thermal conductivity and modulus of these truss architectures at the nano and macro scales are firstly compared with each other, which shows a lower thermal conductivity and a higher shear modulus of the FCC architecture. Properties of the FCC nanotrusses and Diamond trusses are then compared with other materials to show the advantages that these architectures at different length scales can provide for designing materials with unprecedented multifunctional properties.

\subsection{METHODOLOGY}

Building upon the introduced architected SC and FCC CNT-based nano-trusses in reference [23], simplified macroscopic CAD models are created to numerically calculate their effective properties using ANSYS, a commercial finite element analysis package. In the following sections, the steps in preparing the CAD model of the cell architectures at the macro scale and the implemented homogenization scheme are discussed. 


\subsubsection{CAD Modeling OF FCC ANd SC Periodic Truss ArChitectures}

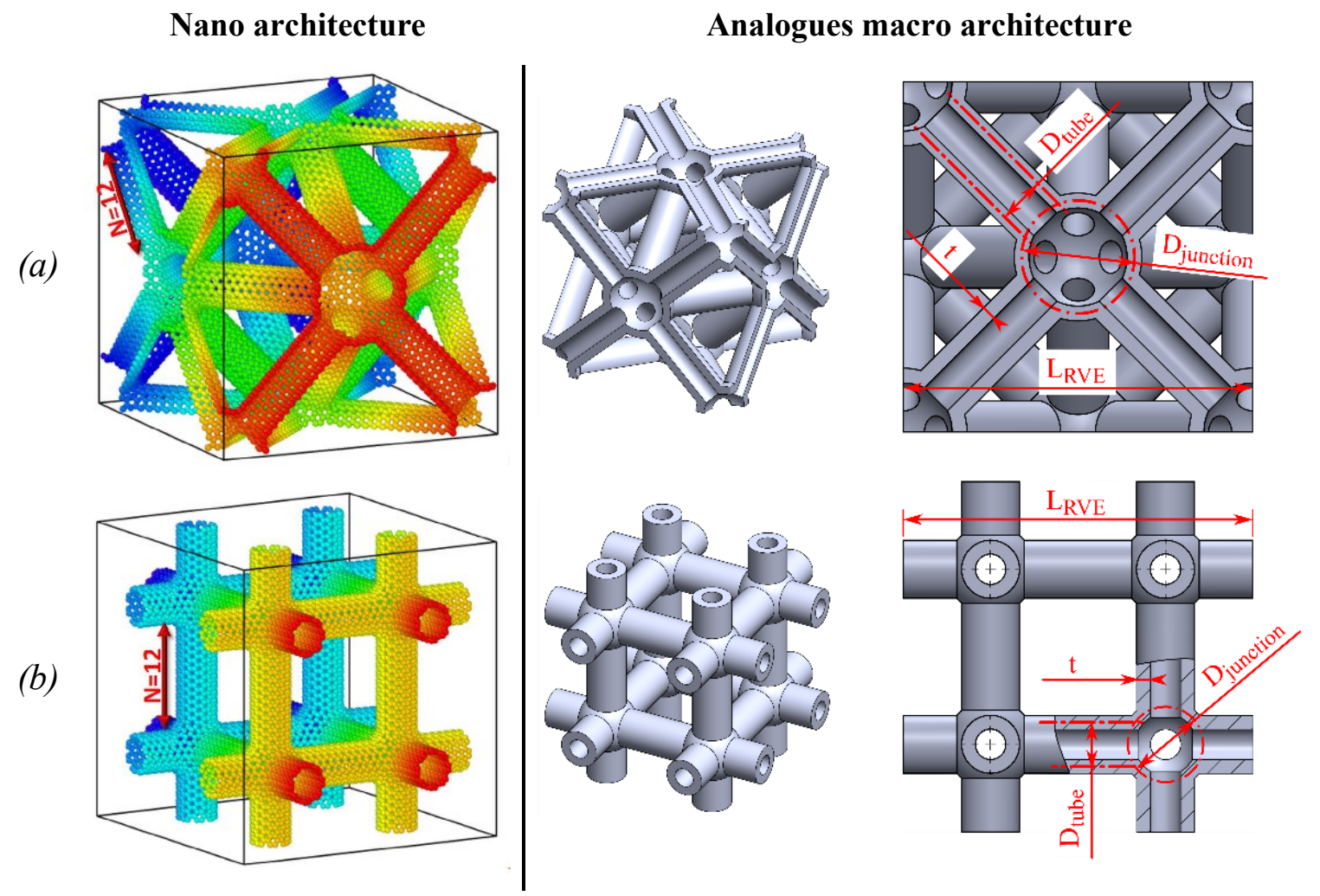

Figure 4.1: (a) FCC and (b) SC truss-like architectures at the nano and macro scales.

Presented in Fig. 4.1, important architectural dimensions that relate the simplified macro scale and the real nano scale architected SC and FCC CNT-based nanotruss materials introduced in reference [23] are first identified. Nano scale dimensions, obtained from the molecular dynamics (MD) model, are then normalized by the length of their corresponding RVE. These relative dimensions, listed in Table 4.1, are defined as:

$$
\begin{aligned}
& t_{r}=t / L_{R V E} \\
& D_{r}^{\text {tube }}=D_{\text {tube }} / L_{R V E} \\
& D_{r}^{\text {junction }}=D_{\text {junction }} / L_{R V E}
\end{aligned}
$$

where, $L_{R V E}, t, D_{\text {tube }}$ and $D_{\text {junction }}$ are geometrical dimensions specified in Fig. 4.1. As shown, compared to the nano-architecture, in the simplified macro scale model CNT parts are replaced by hollow cylinders, and junctions are approximated by hollow spheres. Furthermore, Graphene's layer thickness is considered as the thickness of all members. 
Table 4.1: Relative dimensions of the selected nanotruss architectures

\begin{tabular}{|c|c|c|c|c|c|}
\hline \multicolumn{3}{|c|}{ SC } & \multicolumn{3}{|c|}{ FCC } \\
\hline$\underbrace{0}_{+}$ & 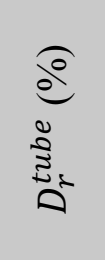 & 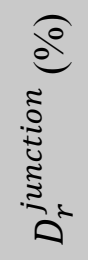 & $\overbrace{+2}^{0}$ & 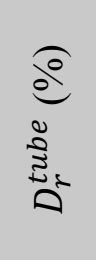 & 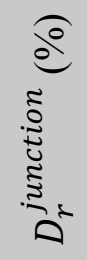 \\
\hline 8.0 & 43.8 & 25.6 & 6.8 & 52.2 & 21.8 \\
\hline 5.5 & 30.0 & 17.6 & 5.4 & 40.9 & 17.1 \\
\hline 3.9 & 21.5 & 12.6 & 4.2 & 32.3 & 13.5 \\
\hline 3.2 & 17.6 & 10.3 & 3.6 & 27.5 & 11.5 \\
\hline 2.7 & 14.8 & 8.7 & 3.2 & 24.0 & 10.1 \\
\hline 2.4 & 12.8 & 7.5 & 2.8 & 21.3 & 8.9 \\
\hline
\end{tabular}

* $\mathrm{N}$ is the tube length parameter used in the MD simulation in [23]

Using the dimensions listed in Table 4.1, the solid models are created in SolidWorks and saved to parasolid format $(. x+t)$ in order to later being imported to ANSYS APDL environment. Relative densities of these CAD models, as measured in Solidworks, are listed in Table 4.2.

Table 4.2: Relative densities of the simplified macroscopic Diamond-truss architectures

\begin{tabular}{|c|c|c|c|c|c|c|c|}
\hline & \multicolumn{6}{|c|}{$\mathbf{N}$} \\
\hline & & 3 & 7 & 12 & 16 & 20 & 24 \\
\hline \multirow{2}{*}{$\begin{array}{c}\text { Relative density } \\
(\%)\end{array}$} & SC & 38.3 & 23.8 & 14.1 & 10 & 7.4 & 5.7 \\
\hline & FCC & 36 & 28 & 20.2 & 15.8 & 12.6 & 10.3 \\
\hline
\end{tabular}

\subsubsection{NUMERICAL HOMOGENIZATION}

Assuming that a cellular material is a perfectly periodic assembly of a single representative volume element (RVE), by employing periodic boundary conditions and using standard homogenization technique, analysis of only one RVE would be enough for obtaining effective thermal conductivity of the cellular material [16]. In this regards, after CAD modeling and having the RVE in ANSYS APDL environment, periodic mesh is created so for each point on one face of the cubic RVE, there is a corresponding point at the projection of the original point on the opposite face. Periodicity and temperature gradient can then be adopted to the cell boundaries by adding temperature constraints to these pairs. 
Following the standard mechanics homogenization approach, three independent unit temperature gradients along the coordinate axes are applied to the cell in three load cases and Fourier heat conduction is numerically solved for each case. The resultant microscopic temperature gradients inside the RVE are then used to construct the $M^{T}$ tensor which relates the applied thermal gradients on the cell boundaries (equal to the average thermal gradient in a homogenized cell under the same boundary load) and the local thermal gradients at each point by $\nabla T=M^{T} \overline{\nabla T}$. Effective thermal conductivity of the RVE is then calculated using:

$$
\bar{K}_{i j}=\frac{1}{V_{R V E}} \int K_{i k} M_{k j}^{T} d V_{R V E} \quad(i, j, k=1,2,3)
$$

in which $\bar{K}_{i j}$ represents effective thermal conductivity tensor, $K_{i k}$ is thermal conductivity tensor of the base material at each point, and $V_{R V E}$ is the volume of the RVE.

In case of structural analysis, temperature gradients are replaced by unit strains and six independent load cases are considered to calculate the $M^{C}$ tensor which relates local and average strains on the RVE by $\varepsilon_{i j}=M_{i j k l}^{C} \bar{\varepsilon}_{k l}$. Homogenized structural properties are then calculated using:

$$
\bar{C}_{i j k l}=\frac{1}{V_{R V E}} \int C_{i j m n} M_{i j k l}^{C} d V_{R V E} \quad(i, j, k, l, m, n=1,2,3)
$$

where $\bar{C}_{i j k l}$ is the effective stiffness tensor and $C_{i j m n}$ is the local stiffness tensor.

\subsection{RESULTS AND DISCUSSION}

To be able to compare effective properties of the macro scale FCC and SC periodic truss materials with their nano scale counterparts, Diamond, which has comparable properties to those of CNT, is assumed as the solid constituent. Consequently, they are named FCC and SC Diamondtrusses. Material properties of the Diamond are listed in Table 4.3. Contributions of radiation, convection and thermal conduction of the gas inside the cells are also neglected.

Table 4.3: Material properties for Diamond [72]

\begin{tabular}{c|c|c|c}
\hline $\begin{array}{c}\text { Density } \\
\left(\mathrm{kg} / \mathrm{m}^{3}\right)\end{array}$ & $\begin{array}{c}\text { Thermal conductivity (k) } \\
\mathrm{W} / \mathrm{mK}\end{array}$ & $\begin{array}{c}\text { Young's modulus (E) } \\
\text { GPa }\end{array}$ & Poisson's ratio $(v)$ \\
\hline 3520 & 2000 & 1220 & 0.2 \\
\hline
\end{tabular}


Figure 4.2 demonstrates effective thermal conductivity of SC and FCC Diamond truss cellular materials obtained using homogenization approach. For both cell architectures, the rotational symmetries dictate isotropic effective thermal conductivities, therefore, only one trend line is shown for each architecture. The higher thermal conductivity of the SC Diamond truss architecture at the examined densities can be justified by the shorter and thicker pathways for the heat to transfer from one side of the cell to the opposite side. On the other hand, for the FCC Diamond truss architecture, inclined and thin truss links present more resistance for the heat to pass through.

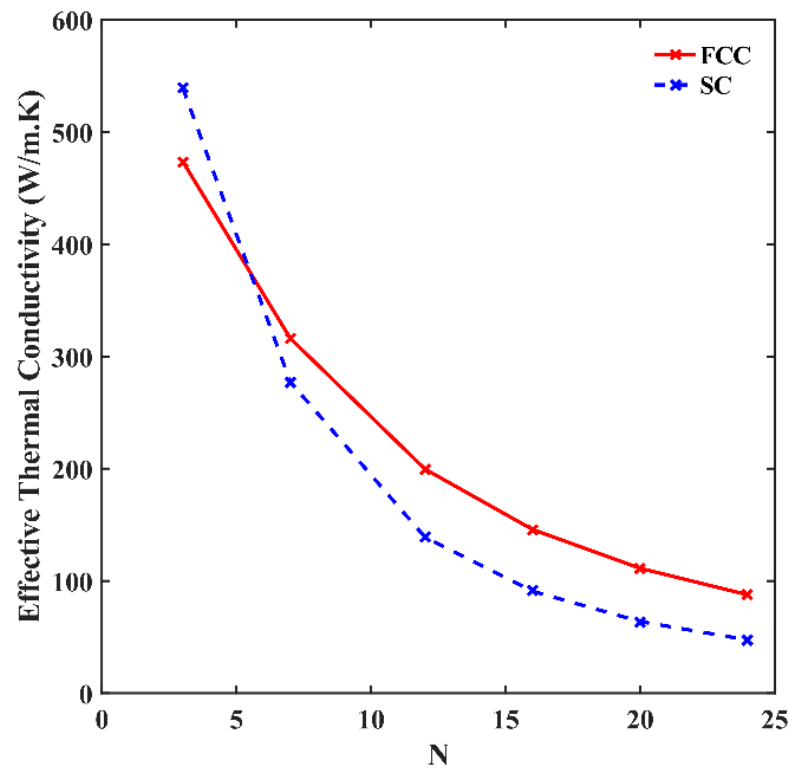

(a)

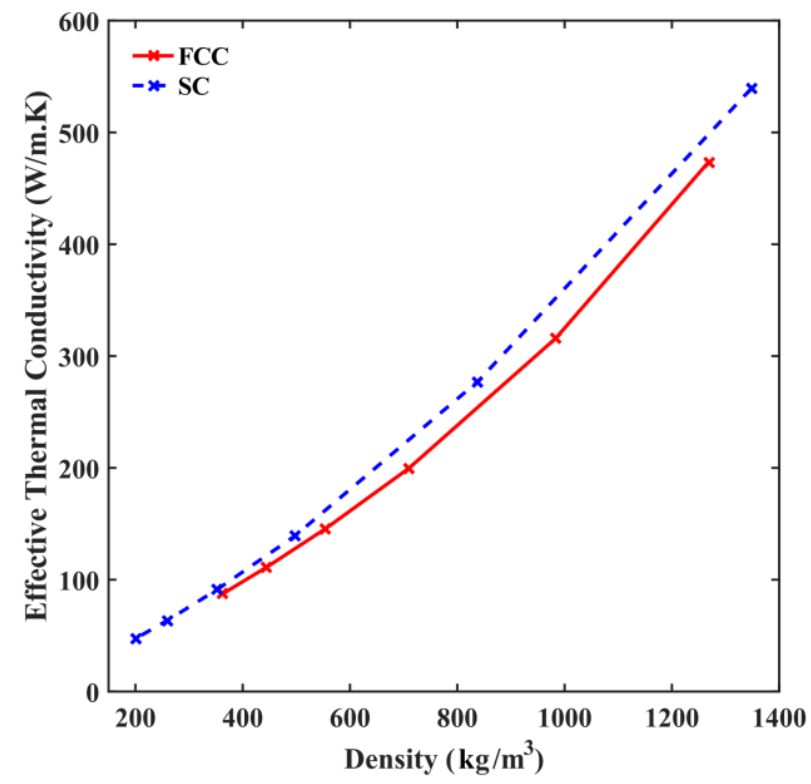

(b)

Figure 4.2: Effective thermal conductivity of SC and FCC Diamond trusses, (a) with respect to $N$, and (b) with respect to density

Homogenized structural properties of the two macroscopic cellular architectures are presented in Fig 4.3. In this case, existing rotational symmetries make them structurally orthotropic and similar to thermal conductivity, one trend line is enough to show each mechanical property along $x, y$ and $z$ directions. Likewise, each of the two Diamond trusses has equal shear moduli along the main coordinate axis (i.e., $G_{x z}=G_{y z}=G_{x y}$ ) and equal Poisson's ratios (i.e., $v_{x y}=v_{y x}=v_{x z}=$ $\left.v_{z x}=v_{y z}=v_{z y}\right)$. 


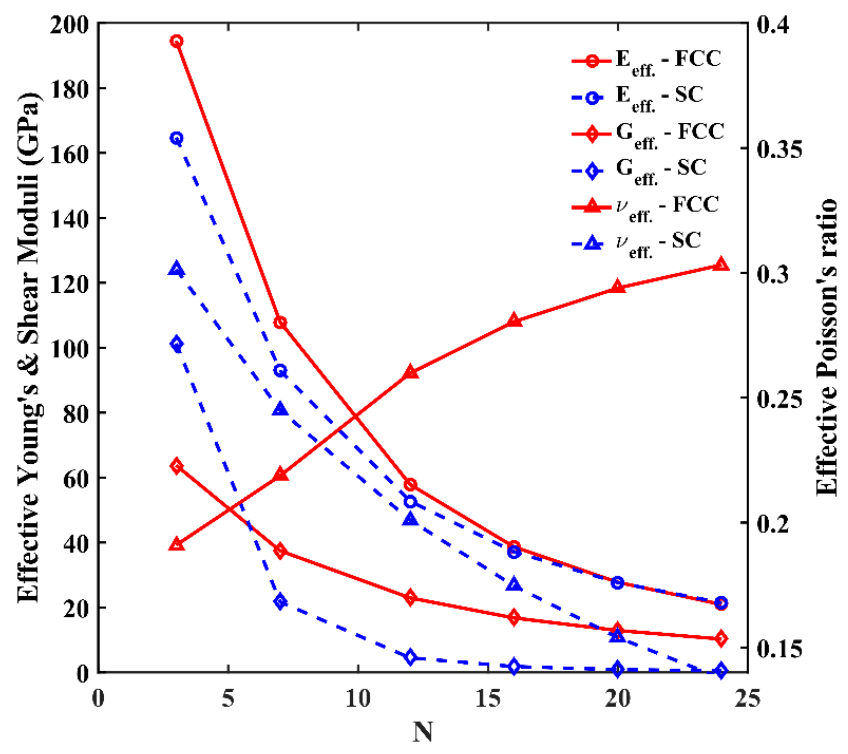

(a)

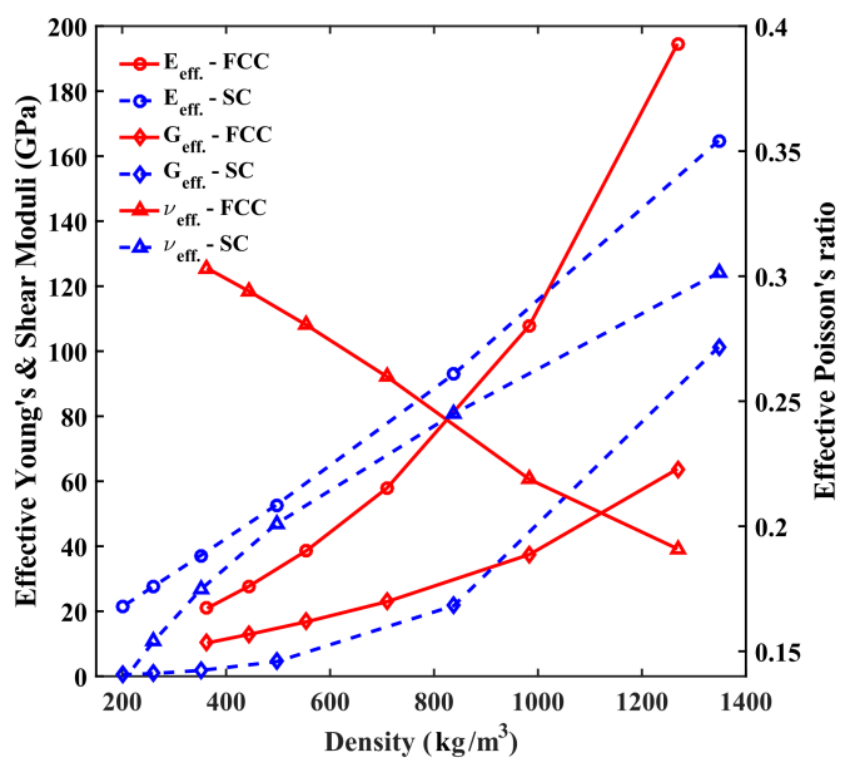

(b)

Figure 4.3: Effective mechanical properties of SC and FCC Diamond trusses along coordinate axes, (a) with respect to $N$, and (b) with respect to density

As shown in Fig. 4.3, increasing $\mathrm{N}$ reduces the effective Young's and shear moduli of both architected materials, which is a consequence of decreased density (please refer to Table 4.2). In addition, unlike FCC truss architecture, Poisson's ratio of the SC diamond truss decreases by increasing N. By increasing N, truss links become longer and the whole cell becomes less rigid, which makes it easier for the truss links to rotate about the junctions compared to when truss links are smaller. The easier rotation of the inclined links of the FCC architecture about the junctions further increases the lateral deformation, leading to increased Poisson's ratio. On the other hand, the truss links of the SC architectures are not inclined with respect to the sides of the RVE and coordinate system, therefore they cannot rotate.

It has also been observed that, while SC architecture has higher effective Young's modulus and smaller effective Shear modulus at densities less than $\sim 1000 \mathrm{~kg} / \mathrm{m}^{3}$, at higher densities, FCC Diamond trusses have higher effective Young's modulus and smaller effective shear modulus. It should be noted that in cases with densities above $1000 \mathrm{~kg} / \mathrm{m}^{3}$ parameter $\mathrm{N}$ is equal to 3 , in which the size of the junction is comparable to the cells size and the architecture does not represent a truss. However increasing N, decreases the relative size of the junction and the structural behavior of the architectures would get more truss-like. Similar to the trusses, in which those with inclined 
links are stronger under shear loading and those with simple cubic arrangement of links are stronger under normal loading, with larger N values FCC architecture are more rigid under shear and more flexible under normal loads.

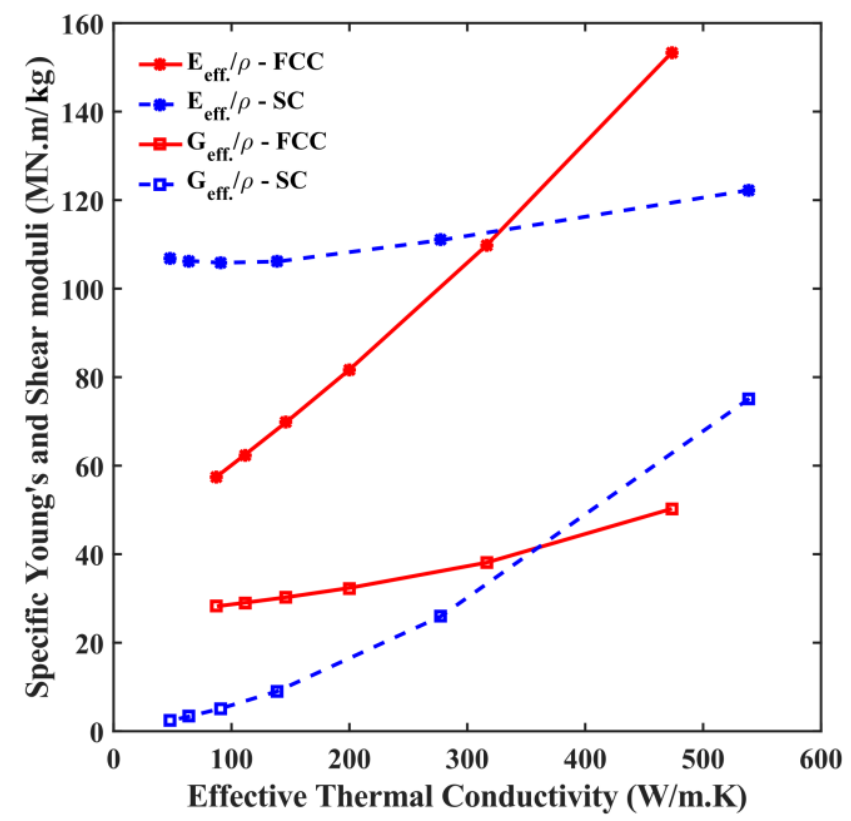

Figure 4.4: Specific effective moduli of FCC and SC diamond truss architectures with respect to their effective thermal conductivities.

Figure 4.4 compares specific moduli of FCC and SC diamond truss architectures, with respect to their effective thermal conductivities. Based on this figure, it is expected that the FCC truss architectures generally perform better where higher thermal conductivity and higher loads are present and the material is expected to be lightweight.

Properties of the SC and FCC diamond trusses are compared with their nano scale counterparts in Fig. 4.5 (as presented in our published manuscript [23]). As demonstrated in Fig. 4.5a, at both macro and nano scales SC truss architectures show higher thermal conductivity compared to FCC architectures; however, effective thermal conductivity of the Diamond trusses is about two orders of magnitude larger than those of the SC and FCC nano-trusses. This is attributed to different phonon scattering mechanisms in the macro and nano scales [23]. Although effective thermal conductivities of the Diamond-truss architectures are significantly higher than those of the nanotrusses, their effective mechanical properties are close (As presented in Fig. $4.5 \mathrm{~b}$ and $4.5 \mathrm{c}$ ). Similar to the macroscopic Diamond trusses, at the nano scale SC nanotrusses have higher 
effective modulus along coordinate axes ([100] direction) and smaller effective Shear modulus (modulus along [110] direction) at densities less than $\sim 1000 \mathrm{~kg} / \mathrm{m}^{3}$, and at higher densities, FCC nanotrusses have higher effective Young's modulus and smaller effective shear modulus.

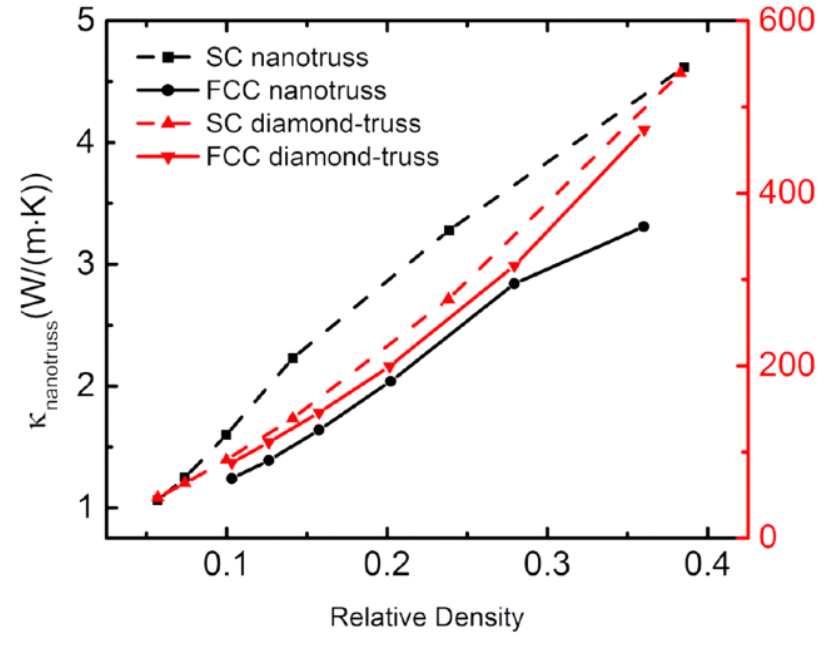

(a)

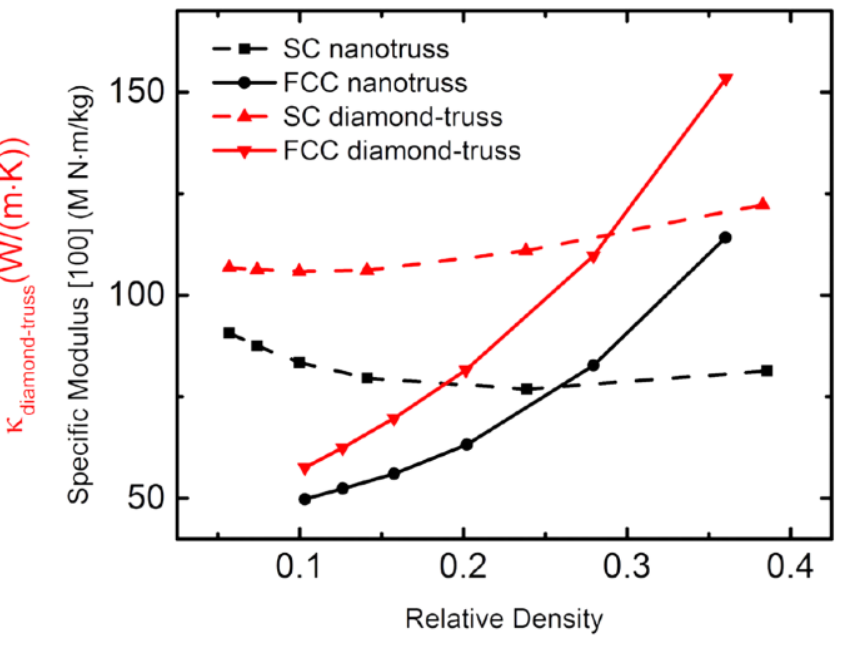

(b)

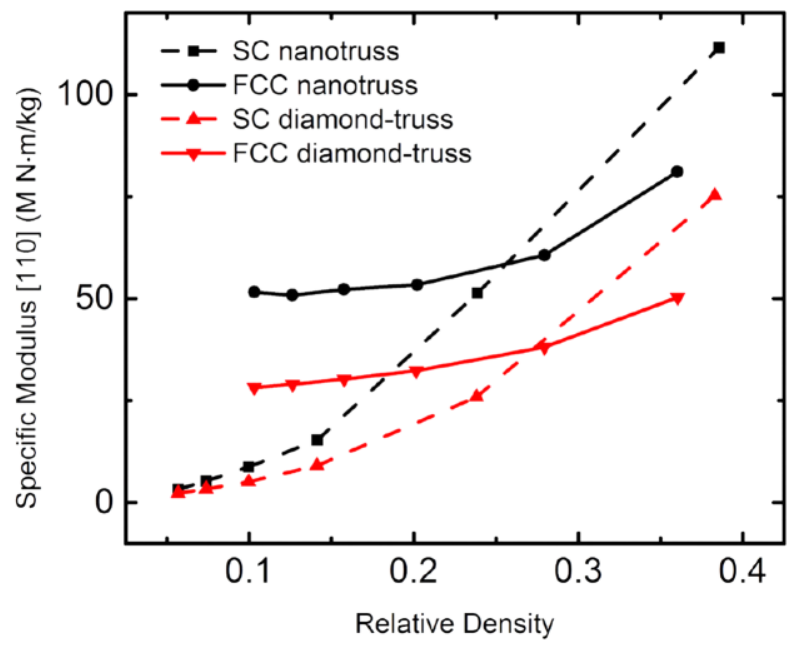

(c)

Figure 4.5: Effective properties of SC and FCC truss architecures at the macro and nano scale as a function of relative density, (a) Effective thermal conductivity, (b) Effective specific modulus along $x$ axis ([100] direction), and (c) Effective Specific shear modulus ([110] direction)

Lastly, effective properties of the FCC nano and Diamond trusses are compared with other materials in Fig. 4.6 (as presented in our published article [23]). As shown, only FCC nanotrusses and some composites are in the domain with specific modulus greater than $40 \mathrm{MNm} / \mathrm{kg}$ and thermal conductivity lower than $5 \mathrm{~W} / \mathrm{mK}$. Considering the thermal stability and extraordinary 
electronic properties of FCC nanotrusses, these cellular materials can be used as multifunctional mechanically robust insulators. Although mechanical properties of the FCC Diamond trusses and nanotrusses are comparable, thermal conductivity of the FCC Diamond trusses are much higher than the FCC nanotrusses, due to different heat transfer mechanisms in different length scales and size-dependency of the thermo-mechanical properties at the nanoscale.

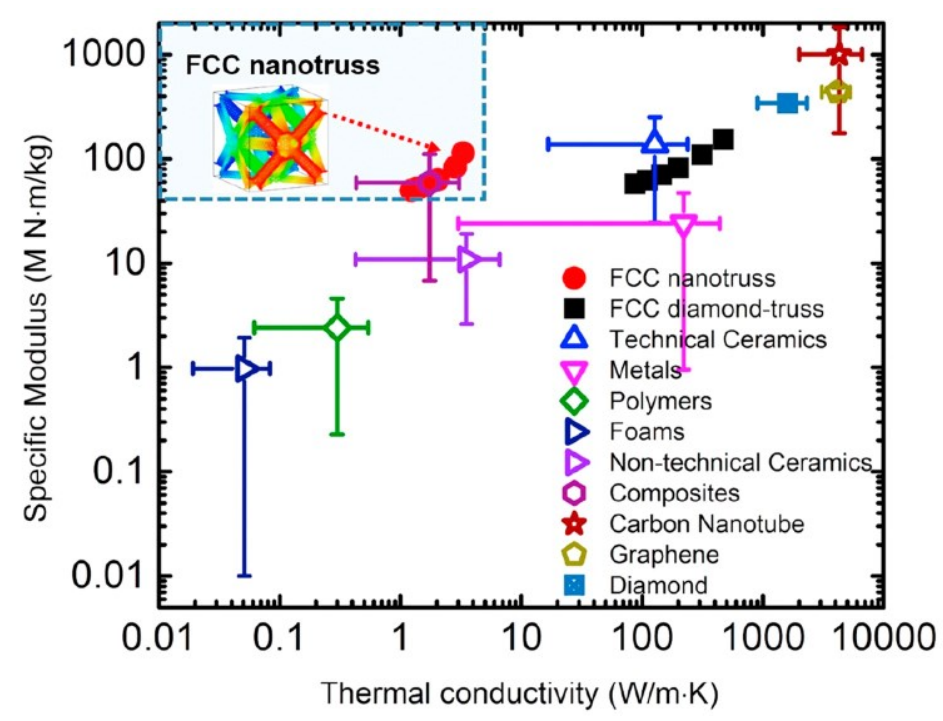

Figure 4.6: Specific modulus ([100] direction) of nano and macro scale SC and FCC trusses with respect to effective thermal conductivity compared to other materials (as reported in [23])

\subsection{CONCLUding Remarks}

In this work, to investigate the length-dependency of the effective properties of the SC and FCC nanotruss architecture, simplified macroscopic analogues are modeled using the relative dimensions of the nano scale cells and their effective thermal and mechanical properties are obtained using standard homogenization. While effective thermal conductivities of these truss-like architectures at the nano and macro scales are shown to be significantly different, their mechanical properties are almost similar. These findings shed light on the optimum design of multifunctional cellular metamaterials, through multiscale designing of the underlying cellular architectures. 


\section{Summary, CONClusion ANd SugGeSted Future Works}

Inspired by the excellent multifunctional performances of cellular biomaterials such as wood, this thesis attempts to study the effective thermal conductivity of architected cellular metamaterials by implementing numerical homogenization scheme, by developing new mathematical methods, and by introducing new cell architectures. Studies are initially focused on 2D lattices with single pores. By investigating square cells with a wide variety of supershape pores with different relative densities, pore aspect ratios and rotation angles, effects of different pore topologies on the effective thermal conductivity of 2D cellular materials are studied. The results clearly show the possibility of creating cellular architectures with anisotropic thermal conductivity. Furthermore, by spatially arranging these architected cells and making an FGCM, the possibility of guiding the heat flow and controlling temperature distribution within a part is demonstrated.

With the understandings from the analysis of $2 \mathrm{D}$ cells, the rest of the thesis focuses on $3 \mathrm{D}$ periodic cellular architectures. First by thickening the previously defined 2D cells and positioning them in a predefined alignment on the faces of a cubic cell, 3D thin-walled open lattices are introduced. A mathematical model is then derived to calculate the effective thermal conductivity of these novel lightweight advanced metamaterials using the data acquired from 2D studies; the homogenized results are also compared with the result of 3D standard mechanics homogenization. The comparison indicates that although thickened 2D cells are placed perpendicular to each other and heat flow within one is seemingly independent of the others, pores of one side of this hollow cube can actually affect the heat transfer through the other sides. Effective thermal conductivity of thin-walled open lattices are also compared with those of TPMS-based Shellular materials. To enhance the achievable thermal conductivity of Shellular architecture, holes with initial 2D lattice arrangement are conformally mapped on the underlying TPMS and subsequently trimmed. The perforated TPMS is then thickened to make the conformally perforated Shellular material (CPSM). Similar to the concept of hierarchical materials, with the addition of mapped holes at a smaller length scale onto the Shellular materials, it would be possible to adjust their thermal properties, although hole shapes and their 2D lattice arrangement will inevitably deform during the mapping process. The effective thermal conductivity of the P-type CPSMs with circular and square holes indicates that by increasing the number of holes, effects of distortion become less noticeable and thermal conductivity converges to the multiplication of effective properties of the underlying 
thermally isotropic 2D and 3D architectures. While it is assumed that all geometrical features of the CPSM are at the continuum length scale, at the nano scale, materials exhibit different behaviour as a result of different physics governing a material's structural and thermal properties at different length scales. To show this length dependency, macroscopic analogues of some CNT-based nanotrusses with FCC and SC arrangements are modeled in SolidWorks and their effective properties are obtained using the standard mechanics homogenization scheme. The properties of the macroscopic scale analogues are compared with those of the original architecture at nano scale (obtained by others using MD simulation), which provides a better understanding of the exceptional multifunctionality of cellular materials at multiple length scales.

The novel cellular architectures introduced in this thesis, the presented mathematical models and the in-depth analyses of the numerical results show many advantages of cellular materials compared to conventional solids. While findings provided in this thesis shed lights on the design aspects of using architected cellular metamaterials for thermal applications, the 3D printed sample architectures clearly show their possible manufacturability through additive manufacturing.

\section{SugGeSTED Future WORKS}

Studies presented in this thesis are performed under certain assumptions, some of which are used to simplify the complex phenomenon involved in thermal behaviour of cellular materials and some are to limit the scope of the work so that it can be completed within the time frame of a master's degree. As a result, to further expand the scope of this thesis for future studies, the followings can be investigated:

- Considering the contribution of convection and radiation on the overall thermal conduction through the cellular material

- Comparing thermal conductivity of gas filled open and closed cell cellular metamaterials at different length scales

- Considering multi-material cellular architectures and the effects of imperfect thermal bonding between them 
- Investigating the effect of changing temperature on the effective thermal conductivity of architected cellular materials made from a base material with temperature-dependant thermal conductivity

- Research on the effective thermal conductivity of CPSMs based on 2D cellular architectures with anisotropic 2D effective thermal conductivities

- Investigating the accuracy of the theoretical findings of this thesis through experimental studies on thermal conductivity of architected cellular metamaterials 


\section{REFERENCES}

1. Gibson, L.J. and M.F. Ashby, Cellular Solids: Structure and Properties. 1999: Cambridge University Press.

2. Pabst, W., T. Uhlírová, E. Gregorová, and A. Wiegmann, Young's modulus and thermal conductivity of closed-cell, open-cell and inverse ceramic foams - model-based predictions, cross-property predictions and numerical calculations. Journal of the European Ceramic Society, 2018. 38(6): p. 2570-2578.

3. Öchsner, A. and T. Fiedler, Effective Thermal Properties of Hollow-Sphere Structures: A Finite Element Approach, in Cellular and Porous Materials. 2008, Wiley-VCH Verlag $\mathrm{GmbH} \&$ Co. KGaA. p. 31-71.

4. Hamer, J., Honeycomb structure and its application to the Concorde rudder. Composites, 1971. 2(4): p. 242-245.

5. Akbarzadeh Shafaroudi, A. and S. Rankohi, Multifunctional and Multiphysics Materials As Load-bearing Structural Components, in Annual Conference of the Canadian Society for Civil Engineering: Resilient Infrastructure. 2016: London. p. 1-9.

6. Evans, A.G., J.W. Hutchinson, and M.F. Ashby, Multifunctionality of cellular metal systems. Progress in Materials Science, 1998. 43(3): p. 171-221.

7. She, W., G. Zhao, D. Cai, J. Jiang, and X. Cao, Numerical study on the effect of pore shapes on the thermal behaviors of cellular concrete. Construction and Building Materials, 2018. 163: p. 113-121.

8. Gasia, J., L. Miró, and L.F. Cabeza, Materials and system requirements of high temperature thermal energy storage systems: A review. Part 2: Thermal conductivity enhancement techniques. Renewable and Sustainable Energy Reviews, 2016. 60: p. 15841601.

9. Zhao, C.Y., Review on thermal transport in high porosity cellular metal foams with open cells. International Journal of Heat and Mass Transfer, 2012. 55(13): p. 3618-3632.

10. Orenstein, R.M. and D.J. Green, Thermal Shock Behavior of Open-Cell Ceramic Foams. Journal of the American Ceramic Society, 1992. 75(7): p. 1899-1905.

11. Volz, S., J. Shiomi, M. Nomura, and K. Miyazaki, Heat conduction in nanostructured materials. Journal of Thermal Science and Technology, 2016. 11(1): p. JTST0001JTST0001.

12. Ranut, P., On the effective thermal conductivity of aluminum metal foams: Review and improvement of the available empirical and analytical models. Applied Thermal Engineering, 2016. 101: p. 496-524.

13. Courant, R. and D. Hilbert, Methods of Mathematical Physics. 2008: Wiley-VCH Verlag GmbH. I-XXII.

14. Singh, R., Thermal Conduction Through Porous Systems, in Cellular and Porous Materials. 2008, Wiley-VCH Verlag GmbH \& Co. KGaA. p. 199-238.

15. Singh, R., Predictions of Effective Thermal Conductivity of Complex Materials, in Heat Transfer in Multi-Phase Materials, A. Öchsner and G.E. Murch, Editors. 2011, Springer Berlin Heidelberg: Berlin, Heidelberg. p. 235-273.

16. Hollister, S.J. and N. Kikuchi, A comparison of homogenization and standard mechanics analyses for periodic porous composites. Computational Mechanics, 1992. 10(2): p. 7395. 
17. S Bakhvalov, N. and G. Panasenko, Homogenisation: Averaging processes in periodic media. . 1989.

18. Belova, I.V. and G.E. Murch, Thermal Properties of Composite Materials and Porous Media: Lattice-Based Monte Carlo Approaches, in Cellular and Porous Materials. 2008, Wiley-VCH Verlag GmbH \& Co. KGaA. p. 73-95.

19. Fiedler, T., I.V. Belova, A. Rawson, and G.E. Murch, Optimized Lattice Monte Carlo for thermal analysis of composites. Computational Materials Science, 2014. 95: p. 207-212.

20. Pabst, W., T. Uhlírová, E. Gregorová, and A. Wiegmann, Young's modulus and thermal conductivity of model materials with convex or concave pores - from analytical predictions to numerical results. Journal of the European Ceramic Society, 2018. 38(7): p. 2694-2707.

21. Uhlírová, T. and W. Pabst, Conductivity and Young's modulus of porous metamaterials based on Gibson-Ashby cells. Scripta Materialia, 2019. 159: p. 1-4.

22. Fiedler, T., A. Öchsner, I.V. Belova, and G.E. Murch, Recent Advances in the Prediction of the Thermal Properties of Syntactic Metallic Hollow Sphere Structures. Advanced Engineering Materials, 2008. 10(4): p. 361-365.

23. Zhang, C., A. Akbarzadeh, W. Kang, J. Wang, and A. Mirabolghasemi, Nano-architected metamaterials: Carbon nanotube-based nanotrusses. Carbon, 2018. 131: p. 38-46.

24. Uhlírová, T. and W. Pabst, Thermal conductivity and Young's modulus of cubic-cell metamaterials. Ceramics International, 2019. 45(1): p. 954-962.

25. Chen, Y., Z. Jia, and L. Wang, Hierarchical honeycomb lattice metamaterials with improved thermal resistance and mechanical properties. Composite Structures, 2016. 152: p. 395-402.

26. Schaedler, T.A. and W.B. Carter, Architected Cellular Materials. Annual Review of Materials Research, 2016. 46(1): p. 187-210.

27. Baillis, D. and R. Coquard, Radiative and Conductive Thermal Properties of Foams, in Cellular and Porous Materials. 2008, Wiley-VCH Verlag GmbH \& Co. KGaA. p. 343384.

28. Belova, I.V., C. Veyhl, T. Fiedler, and G.E. Murch, Analysis of anisotropic behaviour of thermal conductivity in cellular metals. Scripta Materialia, 2011. 65: p. 436-439.

29. Fiedler, T., M.A. Sulong, M. Vesenjak, Y. Higa, I.V. Belova, A. Öchsner, and G.E. Murch, Determination of the thermal conductivity of periodic APM foam models. International Journal of Heat and Mass Transfer, 2014. 73: p. 826-833.

30. Kumar, P., F. Topin, and J. Vicente, Determination of effective thermal conductivity from geometrical properties: Application to open cell foams. International Journal of Thermal Sciences, 2014. 81: p. 13-28.

31. Montazerian, H., E. Davoodi, M. Asadi-Eydivand, J. Kadkhodapour, and M. SolatiHashjin, Porous scaffold internal architecture design based on minimal surfaces: A compromise between permeability and elastic properties. Materials and Design, 2017. 126: p. $98-114$.

32. Wang, W., X. Yang, B. Han, Q. Zhang, X. Wang, and T. Lu, Analytical design of effective thermal conductivity for fluid-saturated prismatic cellular metal honeycombs. Theoretical and Applied Mechanics Letters, 2016. 6: p. 69-75.

33. Lu, Z., Q. Wang, X. Li, and Z. Yang, Elastic properties of two novel auxetic 3D cellular structures. International Journal of Solids and Structures, 2017. 124: p. 46-56.

34. Schaedler, T.A., A.J. Jacobsen, A. Torrents, A.E. Sorensen, J. Lian, J.R. Greer, L. Valdevit, and W.B. Carter, Ultralight Metallic Microlattices. Science, 2011. 334(6058): p. 962-965. 
35. Bauer, T.H., A general analytical approach toward the thermal conductivity of porous media. International Journal of Heat and Mass Transfer, 1993. 36: p. 4181-4191.

36. Gong, L., Y. Wang, X. Cheng, R. Zhang, and H. Zhang, A novel effective medium theory for modelling the thermal conductivity of porous materials. International Journal of Heat and Mass Transfer, 2014. 68: p. 295-298.

37. Hashin, Z. and S. Shtrikman, A Variational Approach to the Theory of the Effective Magnetic Permeability of Multiphase Materials. Journal of Applied Physics, 1962. 33(10): p. 3125-3131.

38. Karttunen, A.T., J.N. Reddy, and J. Romanoff, Micropolar modeling approach for periodic sandwich beams. Composite Structures, 2018. 185: p. 656-664.

39. Chul, H.S., L.J. Woo, and K. Kiju, A New Type of Low Density Material: Shellular. Advanced Materials, 2015. 27(37): p. 5506-5511.

40. Abueidda, D.W., R.K. Abu Al-Rub, A.S. Dalaq, D.-W. Lee, K.A. Khan, and I. Jasiuk, Effective conductivities and elastic moduli of novel foams with triply periodic minimal surfaces. Mechanics of Materials, 2016. 95: p. 102-115.

41. Park, G., S. Kang, H. Lee, and W. Choi, Tunable Multifunctional Thermal Metamaterials: Manipulation of Local Heat Flux via Assembly of Unit-Cell Thermal Shifters. Scientific Reports, 2017. 7: p. 41000.

42. Romano, G., K. Esfarjani, D.A. Strubbe, D. Broido, and A.M. Kolpak, Temperaturedependent thermal conductivity in silicon nanostructured materials studied by the Boltzmann transport equation. Physical Review B, 2016. 93(3): p. 035408.

43. Ghasemi, H., A. Rajabpour, and A.H. Akbarzadeh, Tuning thermal conductivity of porous graphene by pore topology engineering: Comparison of non-equilibrium molecular dynamics and finite element study. International Journal of Heat and Mass Transfer, 2018. 123: p. 261-271.

44. Incropera, F.P., Fundamentals of Heat and Mass Transfer. 2006: John Wiley $\backslash \&$ Sons.

45. Matsuura, M., Gielis' superformula and regular polygons. Journal of Geometry, 2015. 106(2): p. 383-403.

46. Mackay, A.L., Periodic minimal surfaces. Nature, 1985. 314: p. 604.

47. Deng, Y. and M. Mieczkowski, Three-dimensional periodic cubic membrane structure in the mitochondria of amoebaeChaos carolinensis. Protoplasma, 1998. 203(1): p. 16-25.

48. Gan, Z., M.D. Turner, and M. Gu, Biomimetic gyroid nanostructures exceeding their natural origins. Science Advances, 2016. 2.

49. Shin, J., S. Kim, D. Jeong, H.G. Lee, D. Lee, J.Y. Lim, and J. Kim, Finite Element Analysis of Schwarz P Surface Pore Geometries for Tissue-Engineered Scaffolds. Mathematical Problems in Engineering, 2012. 2012.

50. Kapfer, S.C., S.T. Hyde, K. Mecke, C.H. Arns, and G.E. Schröder-Turk, Minimal surface scaffold designs for tissue engineering. Biomaterials, 2011. 32(29): p. 6875-6882.

51. Lei, J. and Z. Liu, The structural and mechanical properties of graphene aerogels based on Schwarz-surface-like graphene models. Carbon, 2018. 130: p. 741-748.

52. Maskery, I., L. Sturm, A.O. Aremu, A. Panesar, C.B. Williams, C.J. Tuck, R.D. Wildman, I.A. Ashcroft, and R.J.M. Hague, Insights into the mechanical properties of several triply periodic minimal surface lattice structures made by polymer additive manufacturing. Polymer, 2017. 
53. Baillis, D. and R.m. Coquard, Radiative and Conductive Thermal Properties of Foams. Cellular and Porous Materials: Thermal Properties Simulation and Prediction, 2008: p. 343-384.

54. Collishaw, P.G. and J.R.G. Evans, an Assessment of Expressions for the Apparent ThermalConductivity of Cellular Materials. Journal of Materials Science, 1994. 29(9): p. 22612273.

55. Fiedler, T., I.V. Belova, and G.E. Murch, Theoretical and Lattice Monte Carlo analyses on thermal conduction in cellular metals. Computational Materials Science, 2010. 50(2): p. 503-509.

56. Boldrin, L., F. Scarpa, and R. Rajasekaran, Thermal conductivities of iso-volume centresymmetric honeycombs. Composite Structures, 2014. 113(1): p. 498-506.

57. Van De Walle, W. and H. Janssen, Validation of a $3 D$ pore scale prediction model for the thermal conductivity of porous building materials. Energy Procedia, 2017. 132: p. 225230.

58. Akbarzadeh, A.H., J.W. Fu, L. Liu, Z.T. Chen, and D. Pasini, Electrically conducting sandwich cylinder with a planar lattice core under prescribed eigenstrain and magnetic field. Composite Structures, 2016. 153: p. 632-644.

59. Niknam, H., A.H. Akbarzadeh, D. Rodrigue, and D. Therriault, Architected multidirectional functionally graded cellular plates. Materials \& Design, 2018. 148: p. 188-202.

60. Akbarzadeh, A.H., J.W. Fu, Z.T. Chen, and L.F. Qian, Dynamic eigenstrain behavior of magnetoelastic functionally graded cellular cylinders. Composite Structures, 2014. 116: p. 404-413.

61. Lee, M.G., J.W. Lee, S.C. Han, and K. Kang, Mechanical analyses of "Shellular", an ultralow-density material. Acta Materialia, 2016. 103: p. 595-607.

62. Nguyen, B.D., S.C. Han, Y.C. Jung, and K. Kang, Design of the P-surfaced shellular, an ultra-low density material with micro-architecture. Computational Materials Science, 2017. 139: p. 162-178.

63. Fratzl, P. and R. Weinkamer, Nature's hierarchical materials. Progress in Materials Science, 2007. 52(8): p. 1263-1334.

64. Meng, T.W., G.P.-T. Choi, and L.M. Lui, TEMPO: Feature-Endowed Teichmüller Extremal Mappings of Point Clouds. SIAM Journal on Imaging Sciences, 2016. 9(4): p. 1922-1962.

65. Crane, K., Conformal Geometric Processing. 2013, California Institute of Technology.

66. Gleiter, H., Nanostructured materials: state of the art and perspectives. Nanostructured Materials, 1995. 6(1): p. 3-14.

67. Gleiter, H., Nanostructured materials: basic concepts and microstructure. Acta Materialia, 2000. 48(1): p. 1-29.

68. Vincenzo, A., P. Roberto, F. Marco, M.M. Onofrio, and I. Maria Antonia, Surface plasmon resonance in gold nanoparticles: a review. Journal of Physics: Condensed Matter, 2017. 29(20): p. 203002.

69. Treacy, M.M.J., T.W. Ebbesen, and J.M. Gibson, Exceptionally high Young's modulus observed for individual carbon nanotubes. Nature, 1996. 381: p. 678.

70. Pop, E., D. Mann, Q. Wang, K. Goodson, and H. Dai, Thermal Conductance of an Individual Single-Wall Carbon Nanotube above Room Temperature. Nano Letters, 2006. 6(1): p. 96-100. 
71. Sinha, S., S. Barjami, G. Iannacchione, A. Schwab, and G. Muench, Off-axis Thermal Properties of Carbon Nanotube Films. Journal of Nanoparticle Research, 2005. 7(6): p. 651-657.

72. Spear, K.E. and J.P. Dismukes, Synthetic Diamond: Emerging CVD Science and Technology. 1994: Wiley. 
解 Peace 